University of Louisville

ThinkIR: The University of Louisville's Institutional Repository

Electronic Theses and Dissertations

$12-2014$

\title{
The effect of spinal cord injury on vagal afferents.
}

April N. Herrity

University of Louisville

Follow this and additional works at: https://ir.library.louisville.edu/etd

Part of the Medical Neurobiology Commons, Musculoskeletal, Neural, and Ocular Physiology Commons, and the Orthopedics Commons

\section{Recommended Citation}

Herrity, April N., "The effect of spinal cord injury on vagal afferents." (2014). Electronic Theses and Dissertations. Paper 1741.

https://doi.org/10.18297/etd/1741

This Doctoral Dissertation is brought to you for free and open access by ThinkIR: The University of Louisville's Institutional Repository. It has been accepted for inclusion in Electronic Theses and Dissertations by an authorized administrator of ThinkIR: The University of Louisville's Institutional Repository. This title appears here courtesy of the author, who has retained all other copyrights. For more information, please contact thinkir@louisville.edu. 
THE EFFECT OF SPINAL CORD INJURY ON VAGAL AFFERENTS

By

\author{
April N. Herrity \\ B.S., University of New Mexico, 1998 \\ M.S., University of Louisville, 2011

\begin{abstract}
A Dissertation
Submitted to the Faculty of the

School of Medicine of the University of Louisville

in Partial Fulfillment of the Requirements

for the Degree of
\end{abstract}

Doctor of Philosophy

Department of Anatomical Sciences and Neurobiology

University of Louisville

Louisville, Kentucky

December 2014 
Copyright 2014 by April N. Herrity

All rights reserved 

THE EFFECT OF SPINAL CORD INJURY ON VAGAL AFFERENTS

By

April N. Herrity

B.S., University of New Mexico, 1998

M.S., University of Louisville, 2011

A Dissertation Approved on

December 2, 2014

By the following Dissertation Committee:

Dissertation Director

Dr. Charles H. Hubscher

Dr. Martha E. Bickford

Dr. David S. Magnuson

Dr. Jeffrey C. Petruska

Dr. David P. Stirling 


\section{DEDICATION}

This dissertation is dedicated to

My parents, Mr. Harold J. Herrity, Jr. and Dr. Vishna A. Herrity

My husband, Mohammed

My sons, Zainedean and Taher

My brother, Sean

for their caring support, love, and words of encouragement. 


\section{ACKNOWLEDGEMENTS}

I would like to express my most sincere gratitude to my mentor, Dr. Charles Hubscher for providing me with such a wonderful graduate and training experience. I thank him for the knowledge and wisdom he has shared with me, which I know will be of great benefit throughout my career. My achievements and the completion of this work are attributed to his sincere support and mentorship. He is truly committed to his students' success and I hope that I can offer the same guidance to others someday. I am grateful for my committee members Drs. Martha Bickford, David Magnuson, Jeffrey Petruska and David Stirling who have generously given their time and expertise and I thank them for contributing insightful information throughout my project. I also would like to acknowledge all of our lab members, including Mr. James Armstrong, Mr. Jason Fell and fellow colleagues Drs. Kris Rau and Patricia Ward for their encouragement and support. Without their excellent technical assistance and expertise, I would not be able to complete this work. I would also like to extend my appreciation to the National Institute of Health (NINDS Grant F31NS077750) for supporting me in this research endeavor. 


\section{ABSTRACT \\ THE EFFECT OF SPINAL CORD INJURY ON VAGAL AFFERENTS}

April N. Herrity

December 2, 2014

Spinal cord injury $(\mathrm{SCl})$ is a significant public health concern that leaves patients with a multitude of life-long disabilities. Major complications of SCI apart from paralysis, include deficits in bladder and bowel function. Lower urinary tract dysfunction continues to remain a top priority issue affecting quality of life for this population. The majority of visceral organs receive a dual sensory innervation from both spinal nerves as well as the vagus nerve. Following $\mathrm{SCl}$, the vagus nerve is a potential pathway through which information from regions below the level of a spinal injury can travel directly to the brainstem, bypassing the spinal cord. The effect of SCl on the vagus nerve and the tissue it supplies has not been thoroughly examined. In order to advance bladder management after $\mathrm{SCl}$, a thorough understanding of its neural control following chronic injury is needed to ultimately improve existing therapeutic options, as well as develop novel interventions that take advantage of this extraspinal route. 
The objective of this project was to describe the anatomical, neurochemical, and electrophysiological profiles of vagal innervation of the rat urinary bladder. Initially, the first study identified both single and double-labeled vagal afferents supplying the rat bladder and distal colon in the nodose ganglion (NG). The degree of neural innervation to the colon also was assessed, as a single axon that dichotomizes and innervates both organs can serve an important role for mediating both normal physiological and pathological reflexes.

Following chronic $\mathrm{SCl}$, we evaluated potential plasticity in subsets of NG neurons which contain projections that bypass the spinal cord from visceral organs, including those projections that specifically supply the bladder. Vagal sensory cell bodies displayed an increase in P2X3 expression and a decrease in IB4 binding, which also held true for many neurons innervating the bladder. Bladder-innervating neurons also displayed altered membrane electrophysiological properties, suggesting they are responsive to a chronic spinal injury. Even though $\mathrm{SCl}$ does not directly sever the vagus nerve, our results indicate vagal afferents, including those innervating the bladder, exhibit neurochemical plasticity post-injury that may have implications for visceral homeostatic mechanisms and nociceptive signaling. 


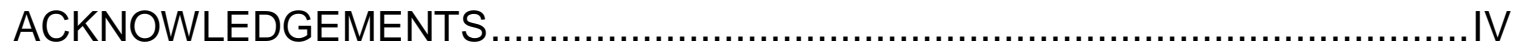

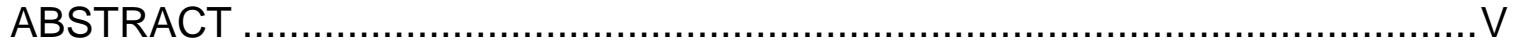

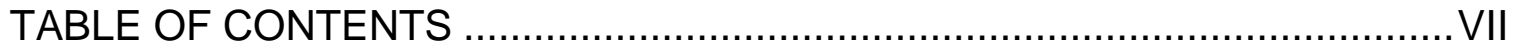

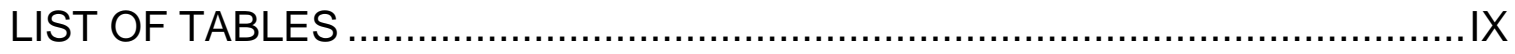

LIST OF FIGURES

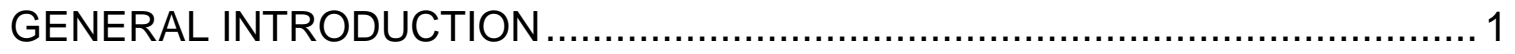

BLADDER DYSFUNCTION FOLLOWING SPINAL CORD INJURY ............................... 2

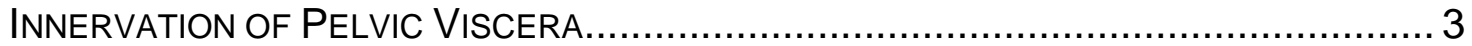

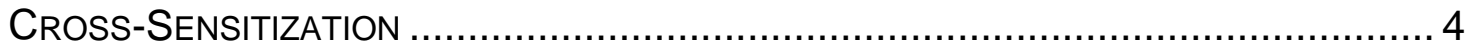

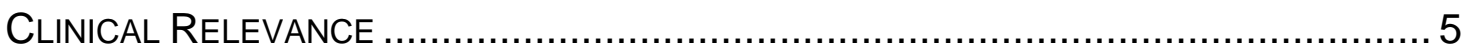

IDENTIFICATION OF BLADDER AND COLON AFFERENTS IN THE NODOSE

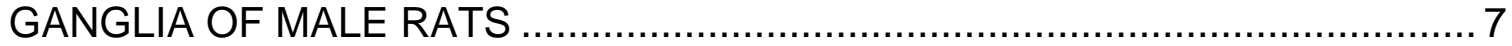

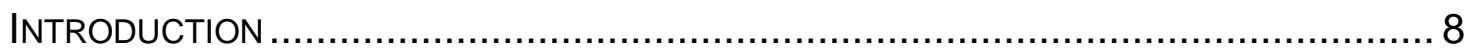

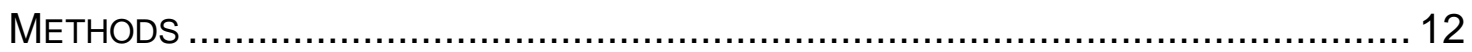

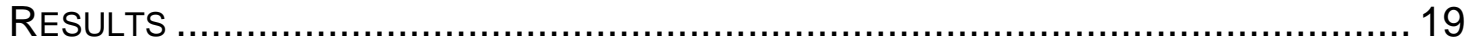

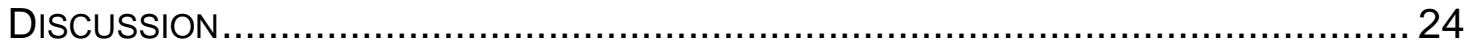

THE EFFECT OF SPINAL CORD INJURY ON THE NEUROCHEMICAL

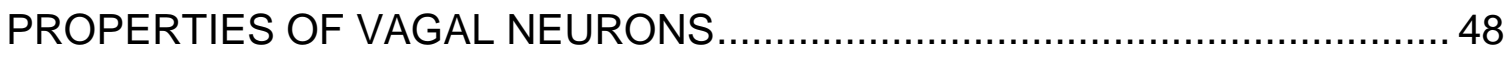

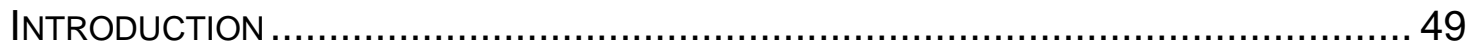

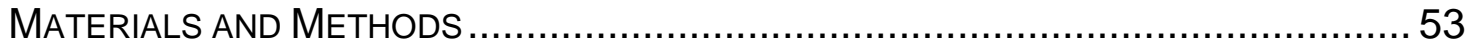

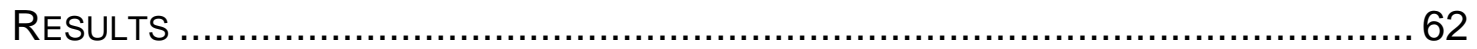

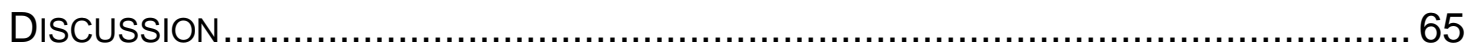


THE EFFECT OF SPINAL CORD INJURY ON THE ELECTROPHYSIOLOGICAL PROPERTIES OF NODOSE GANGLION NEURONS INNERVATING THE

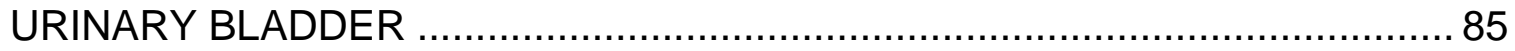

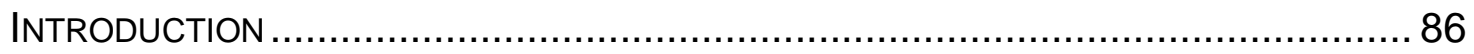

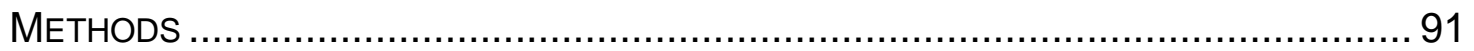

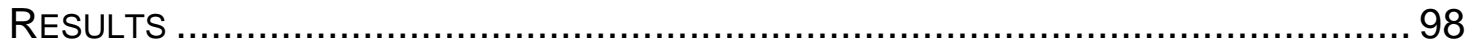

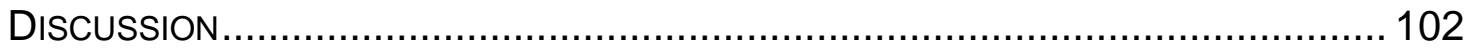

GENERAL DISCUSSION AND FUTURE DIRECTIONS ................................ 117

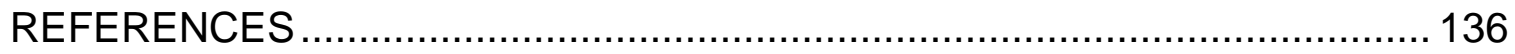

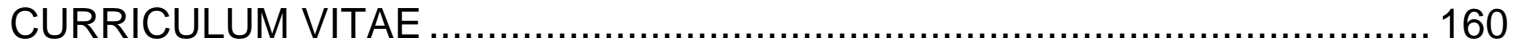




\section{LIST OF TABLES}

TABLE

PAGE

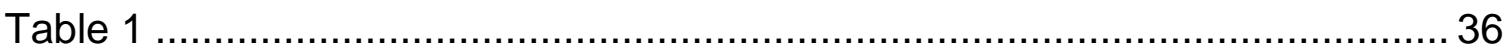

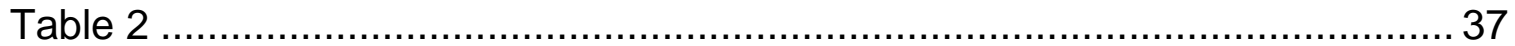

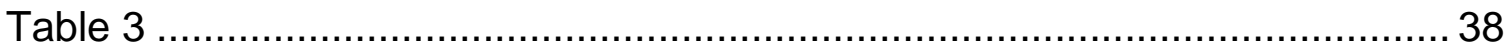

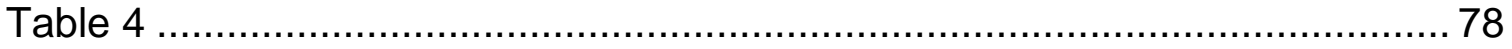

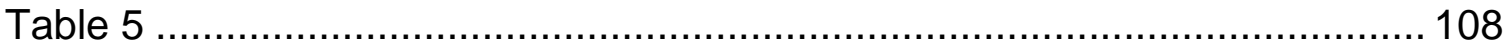




\section{LIST OF FIGURES}

FIGURE

PAGE

Figure 1 Visceral Organ Tracer Injections 39

Figure 2 Percentage of bladder-, colon- and convergent-specific neurons in the

nodose ganglion (NG) 40

Figure 3 Assessment of side differences between NG

Figure 4 Assessment of regional differences in the NG............................... 42

Figure 5 Fluorescent labeling in the NG ................................................... 43

Figure 6 Bladder-, colon- and convergent-specific neurons in the L6 and S1

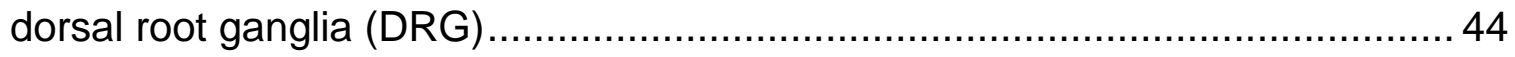

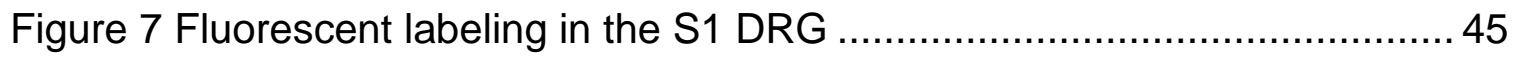

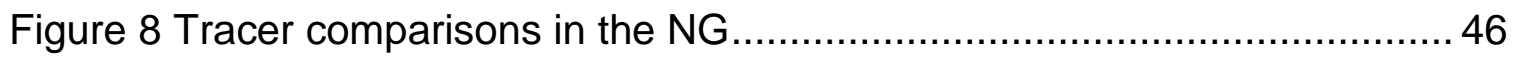

Figure 9 Summary diagram of spinal and vagal innervation to the bladder and

colon

Figure 10 Immunohistochemical representation of P2X3, IB4 and SP in NG

neurons

Figure 11 Quadruple immunohistochemical staining in the NG 80

Figure 12 The effect of SCl on number of NG neurons expressing the individual

markers 81 
Figure 13 The effect of SCI on P2X3 and IB4 in the NG

Figure 14 Effect of SCI on bladder-traced NG neurons ................................... 83

Figure 15 P2X3-ir and IB4 binding in bladder-traced NG neurons after transection

Figure 16 Retrograde tracer injections and identification of bladder NG neurons

Figure 17 Whole cell patch-clamp electrophysiological recording of an NG

bladder afferent

Figure 18 AP characteristics of a NG neuron innervating the bladder

Figure 19 Distribution of non-injured and SCI high and low responders.

Figure 20 Current-Frequency plots of high and low responders in both the intact

and $\mathrm{SCl}$ populations of bladder-innervating NG neurons.

Figure 21 AP profile of SCI high and low responders

Figure 22 The effect of SCI on AP threshold in NG Dil+ neurons...................116

Figure 23 Anatomical innervation of the bladder by the vagus nerve 135 


\section{CHAPTER I}

\section{GENERAL INTRODUCTION}

Major complications of spinal cord injury (SCl), apart from paralysis, include deficits in bowel, bladder and sexual function, autonomic function and chronic pain (Kirchberger et al., 2010). SCl is classified as one the most expensive hospital diagnoses (AHCPR, 1996). Deficits in urological and bowel function rank high in terms of factors affecting quality of life (Anderson, 2004). Development of a neurogenic bladder is a cause of significant morbidity and mortality in the SCI population (Glick et al., 1984, Lynch et al., 2001). To improve bladder and bowel management after $\mathrm{SCl}$, a thorough understanding of its neural control is needed to ultimately aide in improving existing therapeutic options, avoiding unnecessary procedures, and developing novel methods. The outcomes of this project will also provide important information for others suffering from bowel/ bladder dysfunction and visceral pain, including individuals with Multiple Sclerosis, Parkinson's disease, stroke, carcinoma, peripheral nerve insult, interstitial cystitis (IC) and irritable bowel syndrome (IBS). Although the proposed study is directed more toward bladder function, the colon is also being investigated since 1) innervation of the colon by the vagus is known and will 
serve as a complementary comparison to the bladder (Altschuler et al., 1993) and 2) interactions with the circuitry (Basinski et al., 2003, De Wachter and Wyndaele, 2003) including issues surrounding cross-sensitization (Pan et al., 2010) between the bladder and colon in humans make both necessary to study.

\section{Bladder Dysfunction Following Spinal Cord Injury}

Bladder complications post-SCI include over activity of the detrusor muscle leading to incontinence, sustained high pressure within the bladder wall, and sphincter-detrusor dyssynergia which results in a loss of coordination(de Groat and Yoshimura, 2006). SCl individuals often exhibit chronic vesico-ureteral reflux into the renal pelvis, leading to hydronephrosis and ultimately renal failure. Additionally, the backward flow of urine introduces bacteria into the kidneys and can lead to sepsis and hospitalization. In previous years, the leading cause of death in SCI patients was due to renal failure(NSCISC, 2010). However, improvements in urological management have reduced mortality rates, although many treatment options often have low patient compliance (Hansen et al., 2010). Current treatment options for neurogenic bladders include pharmacological agents, catheterizations, surgical procedures, and botulism toxin(Samson and Cardenas, 2007). Even though optimal sterile conditions are sought in bladder care management, most SCI patients develop urinary tract infections, which is the number one medical concern affecting overall health and medical costs (NSCISC, 2010). With the common use of anticholinergic medications, side 
effects such as dry mouth and constipation further exacerbate the underlying urologic dysfunction, making compliance difficult (Benevento and Sipski, 2002).

\section{$\underline{\text { Innervation of Pelvic Viscera }}$}

Anatomical connections to the bladder stem from lumbar sympathetics, sacral parasympathetics and a somatic supply to the external sphincter. However, many studies have shown that much of the pelvic viscera is supplied by both spinal and vagal afferents (Gattone et al., 1986, Jancso and Maggi, 1987, Ortega-Villalobos et al., 1990, Altschuler et al., 1993, Hubscher and Berkley, 1995, Komisaruk et al., 1997, Ersoz and Akyuz, 2004, Hubscher et al., 2004). Evidence of a vagal connection to the pelvic viscera in humans can be demonstrated from the fact that women with functionally "complete" SCI (ASIA criteria) are able to perceive sensations from mechano-stimulation to the vagina and cervix and even respond with orgasms (Komisaruk et al., 1997, Komisaruk et al., 2004). Additionally, patients with functionally "complete" SCI are able to perceive visceral pain from urinary and bowel distention as well as sensations of bladder distention and filling (Wyndaele, 1991, 1997, Ersoz and Akyuz, 2004). Although anatomical evidence of connections between the pelvic viscera and vagus nerve is discussed in the clinical literature pertaining to chronic $\mathrm{SCl}$ cases, no human studies have been performed to determine a vagal connection to the urinary bladder and bowel. Also, anatomy textbooks emphasize that vagal innervations extend only as far as the left colic (splenic) flexure(Agur and Dalley, 
2009). However, earlier neuroanatomical tracing studies in rats have shown that abdominal branches of the vagus nerve innervate all areas of colon, besides the rectum (Altschuler et al., 1993) and portions of the female reproductive tract (uterus, cervix) (Ortega-Villalobos et al., 1990, Hubscher and Berkley, 1995). Evidence of a vagal-bladder connection was found with injection of HRP-WGA into the bladder, which labeled neurons in both spinal ganglia and nodose ganglia (Jancso and Maggi, 1987).

\section{Cross-Sensitization}

Of particular interest is not only the contribution of the vagus to bladder function under normal and pathological conditions, but also aspects of crosssensitization. Central viscero-visceral convergence may explain why some patients experience referred pain or altered sensations in apparently unaffected viscera (Berkley, 2005). In general, cross-talk between pelvic organ systems, such as with the bladder and colon, exist to play a role in normal visceral functioning. For example, distention of the urinary bladder leads to contractions of the external anal sphincter, preventing defecation and allowing for micturition (Basinski et al., 2003). The opposite is true in the process of allowing for defecation while inhibiting micturition. Injury or trauma leading to disruption with the coordination of any of these mechanisms can cause the injured organ to affect the functionality of the non-injured one (Pezzone et al., 2005, Kaddumi and Hubscher, 2006, Malykhina, 2007). If insult to central sources occurs, such as in the case of $\mathrm{SCl}$, there is a potential that alternative inputs to visceral organs may 
become more involved, demonstrating a role in plasticity. Inflammation of the bladder itself has been shown to increase afferent excitability that can elicit painful sensations (Sengupta and Gebhart, 1994). In studies with non-injured humans and rats, both bladder-to-colon and colon-to-bladder cross-sensitization is linked with painful sensations (Alagiri et al., 1997, Pezzone et al., 2005, Theoharides et al., 2008). Therefore, characterizing, using immunohistochemical techniques, the histochemical phenotype of vagal afferents involved in the bladder or colon circuitry and their expression of injury-responsive cellular markers (GS-I-B4, P2X3, SubP) following chronic SCl will provide more insight into the role vagal afferents may play mediating visceral sensation.

\section{Clinical Relevance}

Although afferent innervation of most viscera stems from both spinal and vagal neurons, it is typically thought that nociceptive impulses are mediated through the spinal component (Knowles and Aziz, 2009). However, following injury or irritation, vagal afferents are reported to encode stimuli well into the potentially noxious range which could contribute to visceral hypersensitivity (Bielefeldt et al., 2005). Once clearly identified, these connections can be targeted as possible therapeutic pathways, improving bladder voiding and continence as well as altered sensations. In terms of the overall clinical relevance, vagal nerve stimulation has been shown to be a successful alternative treatment for conditions such as refractory epilepsy (Labar et al., 1999), depression (Cristancho et al., 2011) and even systemic inflammation (Tracey, 
2007). Therefore, understanding the interconnectedness of the vagus nerve and the role it may play in pathological conditions signifies that there may be mechanisms involved in our capability of targeting of the vagus nerve therapeutically to begin to address bladder/bowel dysfunction. Given that many pelvic pain conditions involve organ cross-sensitization mechanisms, our work defining the nature of multi-organ-innervation of single nodose ganglion neurons may provide us with new insights into their co-morbities. 
CHAPTER II

IDENTIFICATION OF BLADDER AND COLON AFFERENTS IN THE NODOSE GANGLIA OF MALE RATS ${ }^{1}$

${ }^{1}$ Herrity AN, Rau KK, Petruska JC, Stirling DP, Hubscher CH (2014) Identification of bladder and colon afferents in the nodose ganglia of male rats. J Comp Neurol 522:3667-3682. 


\section{$\underline{\text { Introduction }}$}

Neural innervation of the lower urinary tract to/from the spinal cord includes sensory and somato-motor components as well as a lumbar sympathetic and sacral parasympathetic supply. Furthermore, the micturition reflex requires descending input from central sources located in an area of the pons referred to now as the pelvic organ stimulating center (POSC,) since it also coordinates other pelvic organ eliminative functions (Holstege et al., 1979, Holstege and Kuypers, 1982, Holstege et al., 1986, Aguayo and White, 1992, Blok et al., 1997b, Huynh et al., 2013, Beckel and Holstege, 2014). In most species, voiding is initiated when information regarding bladder fullness, conveyed by A- $\delta$ fibers, projects to the pelvic organ spinal relay center (POSRC) (Beckel and Holstege, 2011b, a) located near the dorsal horn of the sacral spinal cord, before ascending to the periaqueductal gray (PAG) (Vanderhorst et al., 1996, Holstege, 2005, Klop et al., 2005), a main relay center in the midbrain. From there, receiving cues from the brain indicating voiding is appropriate (Blok et al., 1997a, Blok et al., 1998), the PAG activates the POSC to initiate micturition (Beckel and Holstege, 2011b). In addition to spinal and supraspinal derived sources of neural innervation, numerous animal studies have shown that many pelvic viscera are supplied also by a non-spinal vagal component (Burden et al., 1983, Gattone et al., 1986, Jancso and Maggi, 1987, Ortega-Villalobos et al., 1990, Altschuler et al., 1993, Hubscher and Berkley, 1995, Komisaruk et al., 
1997, Collins et al., 1999, Ersoz and Akyuz, 2004, Hubscher et al., 2004, Komisaruk et al., 2004). Dual sources of innervation by non-spinal routes that convey sensory information to the brain from visceral organs are likely of high functional significance under pathological conditions including chronic spinal cord injury.

Prior neuroanatomical tracing experiments in normal adult rats have shown that the abdominal branches of the vagus nerve innervate all areas of colon, but not the rectum (Altschuler et al., 1993) and comparative studies in rats receiving a complete spinal cord transection at T8 confirm vagal innervation of the colon (Vizzard et al., 2000). Note that the majority of vagal afferent fibers outnumber efferent fibers by a ratio of 10:1 (Grundy, 2002). The degree of vagal contribution to the bladder, however, has not previously been determined. Furthermore, examining the possible connection between the bladder and colon through the vagus nerve is important since cross-talk between pelvic organ systems is part of normal visceral functioning and therefore, physiologically important for maintaining homeostasis. For example, urinary bladder micturition and defecation occur alternately through mutually-inhibitory reflexes. Distention of the urinary bladder leads to contractions of the external anal sphincter, preventing defecation and allowing for micturition (Basinski et al., 2003). The opposite is true in the process of allowing for defecation while inhibiting micturition. If both organs are distended, micturition occurs prior to defecation since it is thought that voiding should happen in a timelier manner due to the fact 
that prolonged urinary retention could irritate the urothelial lining (De Wachter et al., 2007). The vagus nerve also displays important regulatory cross-talk as seen with the baroreceptor reflex, demonstrating its role in maintaining stable blood pressure and heart rate (Dampney, 1994, Spyer, 1994). Injury or trauma leading to disruption with the coordination of any of a number of vagal- or spinalmediated processes can cause the pathological organ to affect the functionality of the non-injured one through a number of mechanisms: cross-excitatory or dorsal root/axonal reflexes, intraganglionic and interaxonal interactions, axon collateral activation, as well as central sensitization (Malykhina et al., 2004, Pezzone et al., 2005, Kaddumi and Hubscher, 2006, Malykhina et al., 2006, Liang et al., 2007, Malykhina, 2007, Ustinova et al., 2007, Brumovsky and Gebhart, 2010, Pan et al., 2010, Ustinova et al., 2010). Influential effects from one organ to another are evident through convergence of inputs at the primary afferent level as well as at second order neurons and higher integrative centers.

Based on our earlier electrophysiological research on responsiveness of medullary reticular formation neurons to stimulation of abdominal branches of the vagus nerve, we found evidence for non-specific afferent induced plasticity with chemical irritation of the bladder, suggesting that vagal afferents may innervate the lower urinary tract (Kaddumi and Hubscher, 2006, 2007). We therefore sought to determine, through anatomical tracing, if evidence of a vagal neural connection to the bladder exists and to assess the degree of convergence (i.e. dichotomizing afferents - reflective of the direction of information flow) with the 
known vagal supply of the distal colon. We hypothesized that there are distinct subsets of NG neurons innervating the bladder or colon, as well as a subset innervating both visceral structures. For comparison, we simultaneously processed and assessed within the same group of animals spinal ganglia (L1/L2 and L6/S1 dorsal root ganglia (DRG)) that are known to innervate both the bladder and colon in the rat. We hypothesized that a greater degree of bladder, colon, and convergent labeling are present in the L6/S1 DRG compared to the NG. 


\section{Methods}

\section{Animals}

All experimental procedures were carried out according to NIH guidelines and protocols reviewed and approved by the Institutional Animal Use and Care Committee at the University of Louisville School of Medicine. All adult male Wistar rats ( $\mathrm{n}=12$, Harlan Sprague Dawley, Inc, Indianapolis, IN), approximately 250 grams in weight, were individually housed in an animal room with a 12-hour light and dark cycle. They had ad libitum access to water and food (Laboratory Rodent Diet).

Injection of retrograde tracers into the urinary bladder and distal colon

One group of adult male Wistar rats $(n=8)$, anesthetized under ketamine (80mg/kg of body weight) and xylazine $(10 \mathrm{mg} / \mathrm{kg})$, received a ventral/caudal midline peritoneal incision to expose the urinary bladder and distal colon. The bladder was emptied and the abdominal viscera were gently shifted to the side for exposure of the distal colon. Using a protocol previously published (Rau et al., 2007), the fluorescent tracer FAST DiO ${ }^{\mathrm{TM}}$ oil (3,3'-dilinoleyloxacarbocyanine perchlorate, $5 \mathrm{mg}$ dye dissolved in $0.1 \mathrm{ml}$ methanol, Molecular Probes Inc., Eugene, OR) was injected with a dye-dedicated 33-gauge needle coupled to a Hamilton microsyringe (Fisher Scientific, Pittsburgh, PA) into the distal colon wall, followed by a separate dye-dedicated 33-gauge microsyringe injection of FAST 
Dil $^{\mathrm{TM}}$ oil (1,1'-dilinoleyl-3,3,3',3',-tetramethylindo-carbocyanine perchlorate, 5mg dye dissolved in $0.1 \mathrm{ml}$ methanol, Molecular Probes, Inc., Eugene, OR) into the bladder wall. Note that the abbreviations Dil and DiO are used throughout the text/figures/legends to refer to FAST $\mathrm{Dil}^{\mathrm{TM}}$ oil and FAST $\mathrm{DiO}^{\mathrm{TM}}$ oil, individually and respectively, and the term carbocyanine to refer to them collectively. For the distal colon, injections were made 1-3 centimeters rostral to the anus $(10 \mu \mathrm{l}$ volume per animal divided into 10 injections of $1 \mu \mathrm{l}$ each). For the bladder, bilateral injections were made to the trigone and dome $(10 \mu l$ volume per animal divided into 10 injections of 1 ul each). Injections were made into the distal colon prior to bladder injections as to avoid organ to organ contact and potential tracer contamination. For tracer comparisons, a set of additional rats $(n=2,4$ ganglia) received bladder injections of either tetramethlyrhodamine (TMR) (anionic, lysine-fixable, $3000 \mathrm{MW}, 2.0 \%$ concentration in $0.9 \%$ saline, Molecular Probes, Inc., Eugene, OR) or Cholera Toxin Subunit B (CTB)-594 (0.5\% injection, Molecular Probes, Inc., Eugene, OR) and distal colon injections of either Fluorescein Dextran (FD) (anionic, lysine-fixable, 3000MW, 2\% concentration in 0.9\% saline, Molecular Probes, Inc., Eugene, OR) or CTB-488 (0.5\% injection, Molecular Probes, Inc., Eugene, OR). A final subset of rats ( $n=2,4$ ganglia) also received colon-only injections of Dil, utilizing the same protocol above.

Animal body temperature was maintained at $37-40^{\circ} \mathrm{C}$ during surgery via a warm water recirculator (Gaymar, Kent Scientific, Winston-Salem, NC). After each injection, the needle was removed slowly; any leakage was controlled by 
cotton-tipped applicators, and the site was rapidly sealed with n-butyl cyanoacrylate monomer glue (Henkel Consumer Adhesives, Avon, $\mathrm{OH}$ ). After injections were completed, the intestines were rehydrated with 5\% Dextrose Lactated Ringers, the abdominal musculature was sutured closed (Ethicon 4-0 non-absorbable surgical suture), the skin closed with Michel clips (Fine Science Tools, Foster City, CA), and a topical antibiotic (Bacitracin, Actavis Mid Atlantic LLC, Lincolnton, NC) applied. Following surgery, animals were placed on a heating pad and core temperature monitored. They were given subcutaneous injections of ketoprofen (Ketofen, 2.5mg/kg, Fort Dodge Animal Health, Fort Dodge, IA) for analgesia twice a day for 2 days, $0.5 \mathrm{ml}$ of dual penicillin (PenJect ${ }^{\circledR}$, The Butler Company, Columbus, $\mathrm{OH}$ ) single dose peri-operatively as a general prophylactic and 5mg/kg gentamicin (GentaFuse ${ }^{\circledR}$, Butler Schein, Dublin, $\mathrm{OH}$ ) once per day for 5 days to prevent infections. All animals were monitored daily to inspect the surgical incision and identify any changes in an animal's general condition.

\section{Perfusion and Tissue Collection}

Ten days after tracer injection, animals were deeply anesthetized with a ketamine $(80 \mathrm{mg} / \mathrm{kg}$ body weight)/xylazine $(10 \mathrm{mg} / \mathrm{kg})$ mixture and transcardially exsanguinated with heparinized saline, followed by $4 \%$ paraformaldehyde perfusion. Each vagus nerve was identified adjacent to the carotid artery and gently separated from surrounding tissues and traced rostrally to the NG, which 
was excised using surgical microscissors and removed. Superior cervical ganglia were identified on both sides at the bifurcation of the common carotid artery and removed to be used as control tissue. In the same rats, following a dorsal spinal laminectomy and removal of the overlying dura mater, paired L1/L2 and L6/S1 dorsal root ganglia were dissected free. Upon removal, all NG and L1/L2/L6/S1 ganglia were placed immediately in individually labeled tubes containing a cryoprotectant solution of $30 \%$ sucrose/phosphate buffer with $1 \%$ sodium azide at $4^{\circ} \mathrm{C}$ for at least 24 hours. The abdominal cavity and viscera were inspected for potential tracer leakage, followed by the removal of the bladder and distal colon.

\section{Antibody Characterization}

The primary antibodies used for signal enhancement of the TMR and FD tracers in this study are described in Table 1 and have been documented previously in numerous tracing studies (McNeill and Burden, 1986, Angelucci and Sainsbury, 2006, Kowski et al., 2008, Saleem et al., 2008, Borra et al., 2010, deCampo and Fudge, 2013). The antibody against TMR has been analyzed previously for cross-reactivity through the dot-blotting method and was shown not to recognize FD or Cascade Blue dextran amines (Kaneko et al., 1996). As expected, both the polyclonal antibodies against TMR and FD did not recognize cells in control tissue from animals not receiving tracer injections in this study (DRG sections) as well as others (Kaneko et al., 1996, McNeal et al., 2010). 


\section{Cell Quantification}

Ganglia coated in embedding medium were cross-sectioned at $12 \mu \mathrm{m}$ on a Leica CM 1850 cryostat and mounted onto gelatin-coated histological slides (Azer Scientific, Morgantown, PA). In the group that received dextran injections, signal amplification of slide-mounted sections was performed by overnight incubation in primary antisera (Rbt-anti-TMR, Molecular Probes, Inc., Eugene, OR, Cat\# A-6397, RRID: AB_1502299 and Goat-anti-FD, Vector Labs, Burlingame, CA, Cat \# SP-0601, RRID: AB_2307446 ) (see Table 1). Secondary antisera (Jackson Immuno ResearchLaboratory Inc., West Grove, PA) diluted 1:200 and 1:100, respectively, was applied for both primary antisera (anti-rbtTexas Red and anti-goat-FITC).

To view the Dil/DiO labeled sections, imaging was performed using the Nikon Eclipse TiE inverted microscope with NIS Elements software. Initially, images were captured using a 10x lens (APO DIC N1 10x/0.45 NA, Nikon) with consistent exposure times and computationally stitched together to visualize whole ganglion sections. Fluorescent imaging utilized a mercury arc lamp filtered through either a Brightline Tritc-B bandpass filter (543/22nm excitation, 593/40nm emission, 562nm dichroic, Semrock) or through a GFP bandpass filter (470/40nm excitation, 525/50nm emission, 495nm dichroic, Nikon). As previously determined, the individual emission maxima of Dil and DiO are easily separated, facilitating two-color labeling, and were therefore selected as our tracers (Honig and Hume, 1986, Godement et al., 1987, Ragnarson et al., 1992). Across the 
entire ganglion, assembled by automated stitching, counts of all singly-, duallyas well as non-labeled (collectively comprising total neuronal counts) NG and DRG neurons with a visible nucleus and definable soma were made using Nikon Elements software in every $5^{\text {th }}$ section (60 microns apart) to avoid double counting. Note that non-labeled cells were quantified if they met the above criteria and did not exhibit any evidence of punctate fluorescence distributed within the cytoplasm, disqualifying them as bladder-, colon- or convergentspecific neurons. Counts for regional differences (rostral, middle and caudal divisions) of the NG were determined using an anatomical division of the NG made by a laryngeal branch of the vagus nerve as described previously (Bielefeldt et al., 2006b). Positive neuron counts were expressed as a percentage of the total number of neurons (labeled and non-labeled) from within the entire stitched ganglion as well as a percentage of all labeled neurons. A Nikon Eclipse 90i confocal microscope (Plan Apo VC DIC N2 oil 60x, 1.4 NA, 488nm excitation/515 emission, $561 \mathrm{~nm}$ excitation/605nm emission, $1 \mu \mathrm{m}$ step size, 13 passes) with EZ-C1 3.60 software and a Nikon A1R MP+ confocal microscope (Plan Apo $\lambda$ 20x, 0.75 NA, 488nm excitation/525nm emission, 561nm excitation/595nm emission, $0.5 \mu \mathrm{m}$ step size, 47 passes) with Elements software were used to collect high resolution, serial optical sections of NG and S1 DRG neurons, respectively. All channels of the confocal images were contrast enhanced simultaneously for display purposes only. 


\section{Statistics}

Analyses were performed using SPSS v19-20 (IBM, North Castle, NY). Levene's statistic was applied for homogeneity of variances and data are expressed as mean \pm SD. Repeated measures analysis of variance (ANOVA) with a group factor was performed for analyzing side differences in the NG. After finding no significant differences between the right and left sides, bilateral ganglia were averaged (some ganglia were lost due to tissue processing errors). Next, these averaged values were analyzed via one-way ANOVA assessing regional differences in the NG. For cell quantifications and tracer comparisons, data were normalized as percentages of total NG cells and analyzed with a one-way ANOVA, followed by Tukey HSD post hoc t-tests. Two-way ANOVA and Tukey HSD post hoc t-tests were performed for the analysis of DRG ganglion differences and pelvic organ differences. One-way ANOVA followed by Tukey HSD post hoc t-tests were used for section sampling data. An independent t-test was performed for evaluating group differences (Dil vs $\mathrm{DiO}$ ) in colon labeled NG neurons. Statistical significance was defined as $p \leq .05$. 


\section{$\underline{\text { Results }}$}

Nodose Ganglion (NG) Labeling

Use of the retrograde lipophilic tracers, Dil and DiO, revealed a total count of 5516 either singly- or dually-labeled afferents supplying the rat bladder and distal colon in the NG (8 rats; 15 total ganglia). Tracer injections into these two different pelvic organs (Figure 1) resulted in significantly more neurons labeled only from bladder than either only from colon or convergent neurons and significantly more neurons labeled only from colon than convergent neurons (Table 2 and Figure 2). Specifically, bladder labeled afferents in the NG were approximately $40 \%$ or 1.7 times more numerous than those labeled from the distal colon and $66 \%$ or almost 3 times more numerous than those labeled from both organs. Colon labeled afferents (Figure 1B) were approximately $43 \%$ or 1.8 times more numerous than dually-labeled afferents (Figure 2). Of the total number of labeled afferents in the NG, $51.6 \pm 1.9 \%$ were labeled only from the bladder, $31.2 \pm 1.5 \%$ only from colon, while convergent neurons represented $17.2 \pm 1.6 \%$. Retrograde tracing from the bladder and colon did not reveal any obvious somatotopy in terms of side-to-side or intra-NG arrangement (Figures 3 and 4, respectively). A typical example showing labeling of NG neurons traced from the bladder and colon is provided in the Figure 5 confocal. Note that percentages of NG labeling from bladder and colon in a female rat ( $n=2$ ganglia) did not reveal significant differences from the male rats (unpublished observations). 


\section{L6/S1 DRG labeling}

In the same set of male Wistar rats ( $n=8,24$ ganglia), injections of Dil into the wall of the bladder and DiO into the wall of the distal colon revealed singlyand dually-labeled spinal afferents in both L6 and S1 DRG. There were significantly more bladder labeled neurons than colon $(p<.01)$ and convergent neurons $(p<.01)$ and significantly more colon labeled than convergent neurons $(p<.01)$ within the L6 DRG (Table 2). Specifically, bladder labeled afferents within the L6 DRG were approximately $47 \%$ or 1.9 times more numerous than those labeled from the $\mathrm{L} 6$ colon and $95 \%$ or 20 times more numerous than those labeled from both organs. Colon labeled afferents were approximately $91 \%$ or 11 times more numerous than dually-labeled afferents within the L6 ganglia (Figure 6). With respect to only the total number of labeled afferents in the L6 DRG, the bladder represented $(64.3 \pm 2.2 \%)$, the colon represented $(32.5 \pm 2.1 \%)$, while convergent neurons represented $(3.2 \pm 0.5 \%)$.

In contrast to the L6 DRG, the numbers of bladder labeled afferents within the S1 DRG were not significantly different from the numbers of colon labeled afferents and, as a group, colon labeled afferents were not significantly different from convergent afferents ( $p>$.05). However, bladder labeled afferents within the S1 DRG were approximately $78 \%$ or 4.6 times more numerous than those labeled from both organs $(p<.05)$. With respect to only the total number of labeled afferents in the S1 DRG, the bladder represented $(59.7 \pm 3.8 \%)$, the colon represented $(25.7 \pm 2.9 \%)$, while convergent neurons represented $(14.7 \pm 1.9 \%)$. 
Examination of labeled neurons across the ganglia revealed that bladder-specific afferents $(74.1 \%$ or 3.9 times, $p<.01)$ and colon-specific afferents $(82 \%$ or 5.6 times, $p<.01$ ) were more numerous in L6 than those labeled from S1. A typical example showing labeling of traced S1 neurons from the bladder and colon is provided in the Figure 7 confocal image.

Since the majority of labeled afferents were present in L6 compared to S1, specific aspects of the collected data were evaluated (i.e. sampling per section) to determine if there were differences between the two DRG levels. Specifically evaluating bladder afferents, there were significantly more Dil + cells per section in L6 $(23.7 \pm 7.2)$ versus S1 $(6.8 \pm 3.6, p<.05)$, but the total number of cells/section were similar $(128.8 \pm 25.9$ versus $124.1 \pm 34.2)$ between the two ganglia. Overall, L6 had a greater amount of Dil labeling compared to S1 and, therefore, a significantly greater percentage of bladder labeling throughout the ganglion (18.6 $\pm 5.2 \%$ vs $5.4 \pm 2.2 \%, p<.001)$.

\section{L1/L2 DRG Labeling}

In a subset of the same male Wistar rats ( $n=4,15$ ganglia), injections of Dil into the wall of the bladder and DiO into the wall of the distal colon revealed singly- and dually-labeled spinal afferents in both L1 and L2 DRG. In contrast to the L6 and S1 DRG, bladder and colon labeling was uniform throughout the L1 and L2 DRG demonstrating no significant differences within and across the ganglia (Table 2). However, in the L2 DRG bladder and colon labeling was 
significantly greater than convergent labeling (bladder, $2.5 \pm 1.3 \%$ vs $0.2 \pm 0.6 \%$, $p<.001$; colon, $2.1 \pm 0.9 \%$ vs $0.2 \pm 0.6 \%, p=.001$ ). With respect to only the total number of labeled afferents in L1, the bladder represented ( $44.8 \pm 19.5 \%)$, the colon (42.4 $\pm 19.0 \%)$ and convergent neurons $(12.7 \pm 16.6 \%)$. Similar results were found for $L 2$, where the bladder represented ( $50.5 \pm 17.3 \%)$, the colon (44.3 $\pm 15.6 \%)$ and convergent neurons $(5.2 \pm 4.5 \%)$.

\section{Spinal versus non-spinal labeling}

In terms of the total percentage of Dil labeled bladder sensory afferents, $49.8 \%$ are represented by a vagal source (NG) and $50.2 \%$ are represented by a spinal source (43.9\% for L6/S1 and 6.3\% for L1/L2). For example, in comparison with the L6 DRG, which contains the vast majority of bladder-labeled neurons, significantly more Dil + cells/section were found in the NG $(51.3 \pm 23.2$ versus $23.7 \pm 7.2$ in L6) as well as total cells/section $(232.5 \pm 76.7$ for NG versus $128.8 \pm$ 25.9 for L6) (Table 3). However, the proportion of bladder labeled neurons relative to the total population of neurons was similar in the NG relative to L6 $(21.6 \pm 3.9 \%$ vs $18.6 \pm 5.2 \%$, Table 3$)$.

\section{Comparisons with other tracers and controls}

Comparisons with other widely used neuronal tracers were performed to assess the effectiveness of the lipophilic tracers Dil and DiO. We compared NG neuronal labeling following injections of dextrans (TMR-into bladder and FD-into 
colon) and CTB (-594-into bladder and -488-into colon). We did not find any significant differences in the percentage of bladder-specific, colon-specific, and convergent-specific neurons in the NG between dextrans TMR/FD and carbocyanines Dil/DiO. However, there were significantly fewer CTB-bladder labeled neurons in the NG than Dil and TMR and significantly fewer CTB-colon labeled neurons than DiO (Figure 8).

Removal of the superior cervical ganglion, which lies in close proximity to the NG, did not reveal any evidence of punctate labeling in conjunction with any of our tracers (image not shown). Furthermore, results from two additional rats that received Dil injections into the distal colon did not reveal significant differences in colon labeled NG afferents from those animals which received prior DiO injections in the distal colon (Dil, $11.0 \pm 1.8 \%$ vs DiO, $12.9 \pm 2.3 \%$ ). As an additional control, the bladder was sectioned and imaged, to ensure there was no evidence of tracer cross-contamination from the distal colon and indicating that the tracer remained in that organ (image not shown). 


\section{Discussion}

In the present study, we identified both singly- and dually-labeled vagal afferents supplying the rat bladder and distal colon. We found a uniform distribution of labeling throughout all regions of bilateral NG ganglia. Furthermore, considering the proportional distribution of labeled afferents between the NG and DRG, the percentages of bladder vagal afferents were similar to the percentages of bladder spinal afferents in the L6 DRG. This result demonstrates that the vagus nerve makes a substantial contribution to the anatomical connections of the male rat bladder. A summary diagram of the dual innervation to the bladder and colon from both spinal and vagal supplies is provided in Figure 9. Based on our comparisons with other types of tracers, the labeling efficacy of the retrograde tracers Dil and DiO, with no known selective tropism for different types of neurons, was appropriate for these experiments with regard to quantifying cells at the primary afferent level.

\section{Nodose Ganglion Labeling}

Injections of the retrograde tracers Dil and DiO revealed bladder-, colonand convergent-specific neurons in the NG of the male Wistar rat. Anatomically, this suggests that a single vagal afferent can supply either the bladder or colon, but also can dichotomize and supply both organs, thereby providing a mechanism of communication between the two structures. 
Evidence of vagal innervation to the male rat bladder provides a greater level of understanding to the complexity of the neural circuitry of the pelvis. These results are in line with previous animal studies demonstrating that the vagus nerve can indeed project below the transverse colon (splenic flexure), providing sensory innervation to the kidney (Gattone et al., 1986), ovary (Burden et al., 1983) and even portions of the female reproductive tract (uterus, cervix) (Ortega-Villalobos et al., 1990, Hubscher and Berkley, 1995, Collins et al., 1999). Additionally, indication of a possible vagal-bladder connection was noted when injection of horseradish peroxidase (HRP)- wheat germ agglutinin (WGA) into the bladder was performed, in which the authors report subsequently labeled neurons in the NG as well as spinal ganglia (Jancso and Maggi, 1987). Furthermore, our result of $21.4 \%$ of bladder neurons represented in the NG is similar to the percentage of NG neurons labeled from stomach (18\%) (Sakurai et al., 2008), demonstrating that the vagus nerve provides a substantial degree of afferent innervation to the bladder.

The fact that $7.4 \%$ of the neurons in the NG were double labeled demonstrates that through the presence of vagal dichotomizing afferents, convergence of bladder and colon afferents are present at the primary afferent level. The percentage of convergent neurons falls within the range of other studies (DRG neurons) in multiple species (5-27\%) (McNeill and Burden, 1986, Brumovsky and Gebhart, 2010). Dichotomizing spinal bladder/colon afferents have been shown at multiple DRG levels in both the rat, mouse (Keast and De 
Groat, 1992, Christianson et al., 2007) and cat (De Groat, 1987b). The existence of these shared peripheral neural pathways may contribute to some of the bladder/colon co-morbid conditions seen clinically, such as interstitial cystitis and irritable bowel syndrome. Evidence of anatomically traced dichotomizing vagal axons has been reported with dually-labeled gastroduodenal (Zhong et al., 2008) and pancreatic (head and tail) afferents present in the NG (Fasanella et al., 2008). Since the bladder and colon are closely related (stemming from the same embryological origin, having the same spinal innervation peripherally, similar central processing areas, similar functions related to storage and elimination), it is perhaps not surprising that there is evidence of co-innervation and sensory neuron-level cross-talk. The functional relevance of communication between the bladder and colon is thought to aid in maintaining bodily homeostasis (DennyBrown, 1933, Floyd et al., 1978, 1979, 1982, De Wachter and Wyndaele, 2003, Vilensky et al., 2004, De Wachter et al., 2007, Malykhina et al., 2012).

The existence of such shared pathways also provides a means by which pathology in one organ can affect the functionality of an adjacent, healthy organ. For instance, numerous studies in experimental models of different species and in clinical reports (Whorwell et al., 1986a, Whorwell et al., 1986b, Alagiri et al., 1997) demonstrate physiological evidence of cross-organ sensitization between both organs, including effects such as increased urinary frequency, urgency, nocturia, abnormal bladder detrusor muscle contractility and emptying or increased responses to colonic distention at lower than normal pressures (Floyd 
et al., 1978, 1979, 1982, Bouvier et al., 1990, Malykhina et al., 2004, Pezzone et al., 2005, Qin et al., 2005, Bielefeldt et al., 2006a, Lamb et al., 2006, Malykhina et al., 2006, Ustinova et al., 2006, Noronha et al., 2007, Qiao and Grider, 2007, Rudick et al., 2007, Ustinova et al., 2007, Ustinova et al., 2010, Lei and Malykhina, 2012, Lei et al., 2013). It is important to note that convergence through both spinal and/or vagal afferents occurs centrally as well. Neurons in the solitary nucleus and medullary reticular formation, for example, have been shown to receive mechano-sensitive inputs from multiple pelvic organs, demonstrating convergence at the second order neuronal level and beyond (Hubscher and Berkley, 1995, Kaddumi and Hubscher, 2006). Central viscerovisceral convergence likely explains why multi-symptomatic patients experience referred pain or altered sensations in unaffected viscera (Berkley et al., 2005).

\section{L6/S1 and L1/L2 Labeling}

The quantitative results of singly- and dually-labeled spinal afferents supplying the male rat bladder and colon were similar to an earlier study (Christianson et al., 2007) assessing this same outcome for L6/S1 and L1/L2 spinal ganglia in the rat despite using different tracers and in a different strain (Sprague-Dawley). There were no significant differences from the Christianson et al. study in terms of labeling between the bladder, colon and convergent spinal afferents in the L6/S1 ganglia when considering only labeled afferents. Similar findings were also apparent for the L1 and L2 ganglia between studies. Although 
percentages for labeled afferents with respect to all neurons (both labeled and non-labeled) was not reported in that study, the current study indicates significant differences when considering the L6 and S1 separately for this measurement criterion. A similar pattern of labeling in the L6 DRG was found compared to the NG, where there were a greater number of bladder labeled afferents followed by colon and then convergent neurons. In S1, only significantly more bladder than convergent neurons was found. However, L6 still displayed a greater percentage of bladder and colon afferents than S1, indicating a larger degree of bladder/colon information travels through this DRG compared to S1. Even though this study focused on sensory afferents (NG via the abdominal branches of the vagus and L6/S1 via the pelvic nerve) of nerves containing parasympathetic fibers to the bladder, there also are some inputs to thoracolumbar DRG's via the hypogastric nerve which contains sympathetic innervation of the bladder. Note that the L1/L2 DRG results are consistent with a predominant sensory supply via L6/S1 pelvic afferents (Christianson et al., 2007) and even when combined the spinal sources and NG supply are in relatively similar proportions.

The general finding of significantly more bladder versus distal colon labeled afferents in both the NG and DRG may be attributed to surface area differences between the two organs. For example, even though the same circumferential region of the distal colon (1-3cm rostral to the anus) and bladder receive the same injection parameters across all animals, the actual injection area of the distal colon is smaller than the bladder with respect to the entire 
organ and therefore may explain the smaller percentage of distal colon afferents relative to the bladder.

The bladder afferent percentages in the NG were most similar to those found in the L6 ganglia. Despite the fact that the NG exhibited more bladderspecific cells per section as well as more total cells per section compared to L6, there were no significant differences in the overall representation of bladder labeling throughout the two ganglia. These findings are consistent with reports on the percentage of stomach-labeled afferents in the NG, which were also found on average to be similar to the percentage of DRG (T9/10) stomach labeled (18\%) afferents (Sakurai et al., 2008). The proportional similarities of the dual supply of bladder afferents may relate to different functional roles of NG and DRG neurons. For example, it has been hypothesized that spinal afferents are responsible for conveying noxious mechanical information, while noxious chemical stimuli are conveyed via vagal afferents (Schuligoi et al., 1998, Michl et al., 2001, Page et al., 2002, Holzer, 2003, Lamb et al., 2003, Danzer et al., 2004, Holzer et al., 2004, Sugiura et al., 2005, Kaddumi and Hubscher, 2007). Aside from communicating differential stimuli from the periphery, both vagal and spinal projections likely converge in the brainstem. In particular, the midbrain PAG receives spinal afferent information from the lower urinary tract as well as vagal afferent visceral information via projections from the solitary nucleus (Herbert and Saper, 1992, Monnikes et al., 2003, Nishii et al., 2008). The PAG also receives input from various cortical regions which play a role in conscious control such as 
determining when voiding is appropriate (Blok et al., 1997a, Blok et al., 1998, Kuipers et al., 2006); Please refer to (Beckel and Holstege, 2014) for a thorough review). Numerous levels of input and organization help provide fine tune control over complex neural pathways important for conscious control of continence and micturition.

We did not find significant differences between NG colon and convergent afferent labeling compared to that from DRG neurons. A large degree of convergent afferents may be expected for the DRG supply based upon their known roles in multiple eliminative functions, but this was not the case. It should be noted, however, that labeled DRG and NG neurons may not accurately reflect the full extent of the peripheral supply to these pelvic organs as there could be differences in the extent of branching. For example, sacral DRGs have been shown to have 2.3 times the number of peripheral fibers as there are cell bodies (Langford and Coggeshall, 1981).

\section{Organization of the NG Neurons}

Included in the quantification of NG neuronal labeling from the bladder and colon were assessments of potential rostral, middle and caudal regional differences. The NG may exhibit a specialized viscerotopic organization where, potentially, vagal afferents from one organ reside more frequently in one area compared to other regions (Browning and Mendelowitz, 2003, Bielefeldt et al., 2006b). A specific organized distribution is important for the coordination of 
events and reflexes as demonstrated in higher integrative centers (Broussard and Altschuler, 2000, Altschuler, 2001). The solitary nucleus, location of vagal afferent terminals, for example, exhibits a high degree of organization with a distinctive organotypic pattern of inputs. Gustatory information is localized rostrally, while cardiovascular, respiratory and gastrointestinal afferents terminate in the caudal two-thirds of the nucleus (Torvik, 1956, Cottle, 1964, Beckstead and Norgren, 1979, Kalia and Mesulam, 1980). Cell counts in sections from the rostral, middle and caudal NG were analyzed and no significant regional differences were found. The finding of a uniform distribution of labeled bladder/colon/convergent cells in the NG also has been demonstrated for upper digestive afferents in the mouse (Zhong et al., 2008) and rat (Altschuler et al., 1989) as well as for pancreatic afferents in the rat (Sharkey and Williams, 1983). In addition, side to side comparisons between both NG's were made since the left and right vagus nerves are known to play different roles with regard to cardiac function (Randall et al., 1986, Saper et al., 1990, Schachter and Saper, 1998). Importantly, retrograde tracing from the heart (coronary sulcus and anterior interventricular groove) and pancreas reveal asymmetrical vagal distributions in the NG (Sharkey and Williams, 1983, Carobi, 1987, Fasanella et al., 2008, Hayakawa et al., 2011). However, despite the slight anatomical differences in the distribution of the thoraco-abdominal vagus, no side differences were present in the current study for the pelvic viscera supplied by the abdominal branches of the vagus. 


\section{Tracing Controls}

One caveat related to the selection of neuroanatomical tracers is whether multiple fiber types take up and retrogradely transport the tracer, as the goal of this initial study was not only to determine if evidence of vagal innervation of the bladder/colon exists but also to quantify the extent of innervation by the abdominal branches of the vagus nerve. The selection of the carbocyanine retrograde tracers, Fast $\mathrm{Dil}^{\mathrm{TM}}$ and Fast $\mathrm{DiO}^{\mathrm{TM}}$, for the current study was based upon their capability of uptake in all cell types (small, medium, large, unmyelinated, lightly- and myelinated) and a well-documented method for tracing cells from the periphery (Honig and Hume, 1989, Su et al., 1999, Wang and Scott, 1999, Ohtori et al., 2001, Ueno et al., 2001, Gold et al., 2002, Deng et al., 2007, Wang et al., 2007). The additional assessments with other tracers, dextran amines and CTB, allowed us to examine the versatility of the lipophilic tracers we were interested in utilizing and ensure their effectiveness for the current study. Certain HRP conjugates, such as Biotinylated-HRP or WGA-HRP have been shown to have a preference towards larger diameter or smaller diameter, myelinated axons, whereas vagal afferents are comprised of primarily small to medium size, unmyelinated or lightly myelinated axons (Robertson and Arvidsson, 1985, Rivero-Melian and Grant, 1991, Maslany et al., 1992, Zhuo et al., 1997). Cholera toxin subunit B (CTB), a commonly used tracer, may be problematic since it requires the presence of the GM-1 ganglioside for uptake (Heyningen, 1974) and perhaps not all vagal bladder/colon afferents express this 
receptor. This consideration could provide an explanation for why we found significantly fewer CTB-labeled bladder and colon neurons in the NG compared to the other tracers used. It is important to note that the use of dextran amines (TMR/FD), while yielding similar NG cell counts as Dil/DiO, also utilize a different uptake mechanism (active transport) than the lipophilic type (passive diffusion) (Kobbert et al., 2000).

As a control, the superior cervical ganglia were processed for detection of the retrograde dyes. This ganglion is spatially close to the NG, with fibers that run relatively adjacent to the vagus nerve. There was no evidence of any dye transfer between the two ganglia in the present study. In addition, previous studies examining the use of these tracers did not find any complications with tracer leakage or the spread of dye to adjacent organs/neurons as well as spread within the ganglia itself (DRG in this example) (Dang et al., 2005, Dang et al., 2008).

To address any quantification concerns due to potential spectral overlap from the long fluorescent emission tail of DiO that may confound our image analyses, Dil was injected into the distal colon of two additional rats and counts of Dil positive neurons were compared with DiO positive colon neurons in the NG. In agreement with our initial colon counts, similar percentages of colon labeled afferents were found between the two groups, indicating that the tracers could be used reliably together and separated by the emission filter combinations used. Additionally, this finding corroborates the notion of convergent neurons in the NG. Overall, based on our controls, the lipophilic tracers incorporated in this 
study were effective for accurately labeling bladder and colon afferents as well as maintaining stable fluorescence for cell quantification in the NG.

\section{Clinical Relevance}

Current human anatomical texts report that vagal innervation to the viscera terminates at the left colic (splenic) flexure (Agur and Dalley, 2009). Complementary to this understanding, a 2013 review of the female abdominopelvic region indicates this caudal extension of the vagus based upon a drawing from the mid-1800s (Frankenhaüser, 1866), in which the authors additionally state that upon entry into the abdomen, the sympathetic and vagal fibers become "indistinguishably mixed" (Shoja M., 2013). Indirect evidence of a vagal connection to the pelvic viscera in humans is demonstrated from the fact that women with functionally "complete" spinal cord injury (SCl - American Spinal Injury Association criteria) are able to perceive sensations from mechanostimulation to the vagina and cervix and even respond with orgasms (Komisaruk et al., 1997, Komisaruk et al., 2004). Additionally, patients with functionally "complete" SCl are able to perceive visceral pain from urinary and bowel distention as well as sensations of bladder distention and filling (Wyndaele, 1991, Ersoz and Akyuz, 2004). An unanswered question is whether additional tracing studies would reveal a further caudal extension of the vagus in humans. Although anatomical evidence of connections to the pelvic viscera by the vagus nerve is discussed in the clinical literature pertaining to chronic $\mathrm{SCl}$ cases, we 
hypothesize that the vagal pathways exist in the human pelvic cavity, but in terms of generating perception, the fibers are "silent" and re-organize following injury or under certain pathophysiological conditions and thus become evident. Given that the putative role of vagal afferents is relaying homeostatic information, much of which we are unaware, this hypothesis seems plausible. However, vagal afferents show the capability of detecting noxious stimuli, as seen in the lungs for example. Their responses to inflammatory insults evoke defensive responses such as a temporary cessation of breathing, bronchoconstriction, hypotension and coughing, thereby providing the organ with its own potential sense of injury, which fulfills Sherrington's role of a nociceptor (Sherrington, 1906, Coleridge and Coleridge, 1984, Undem et al., 2004). 


\section{Table 1}

Summary of antibodies used

\begin{tabular}{|c|c|c|c|c|}
\hline Target & Immunogen & $\begin{array}{l}\text { Host organism, } \\
\text { clonality, } \\
\text { manufacturer, } \\
\text { catalog No., } \\
\text { Research } \\
\text { Resource } \\
\text { Identifier }\end{array}$ & Dilution & $\begin{array}{l}\text { Specificity } \\
\text { controls }\end{array}$ \\
\hline TMR & $\begin{array}{c}\text { 5-carboxy- } \\
\text { tetramethylrhodamine }\end{array}$ & $\begin{array}{c}\text { Rabbit, polyclonal, } \\
\text { Molecular Probes, } \\
\text { Cat\# A-6397, } \\
\text { RRID: AB_1502299 }\end{array}$ & $1: 6,000$ & $\begin{array}{c}\text { Tissue type } \\
\text { controls did } \\
\text { not produce } \\
\text { perikarya } \\
\text { labeling. }\end{array}$ \\
\hline FD & $\begin{array}{c}\text { Fluorescein } \\
\text { isothiocyanate }\end{array}$ & $\begin{array}{l}\text { Goat, polyclonal, } \\
\text { Vector Labs, Cat\# } \\
\text { SP-0601, RRID: } \\
\text { AB_2307446 }\end{array}$ & $1: 2,000$ & $\begin{array}{c}\text { Tissue type } \\
\text { controls did } \\
\text { not produce } \\
\text { perikarya } \\
\text { labeling. }\end{array}$ \\
\hline
\end{tabular}

TMR-tetramethylrhodamine, FD-Fluorescein Dextran 


\section{Table 2}

Average $( \pm \mathrm{SD})$ number of Dil/DiO-positive neurons counted per ganglion

\begin{tabular}{lccccc}
\hline & Vagal PNS & Spinal PNS & Spinal PNS & Spinal SNS & Spinal SNS \\
\hline & NG & L6 DRG & S1 DRG & L1 DRG & L2 DRG \\
Bladder & $188.5 \pm 92.0$ & $165.8 \pm 64.7$ & $42.9 \pm 28.7$ & $30.7 \pm 42.2$ & $30.1 \pm 17.7$ \\
Colon & $114.3 \pm 52.8$ & $87.9 \pm 49.6$ & $15.8 \pm 9.4$ & $23.9 \pm 24.5$ & $24.1 \pm 11.9$ \\
Convergent & $64.9 \pm 38.6$ & $8.1 \pm 4.7$ & $9.3 \pm 6.1$ & $5.0 \pm 4.4$ & $2.4 \pm 1.9$ \\
$\begin{array}{lcccc}\text { Total } \\
\text { Labeled }\end{array}$ & $367.7 \pm$ & $261.8 \pm$ & $68.0 \pm 33.0$ & $59.6 \pm 66.2$ & $56.6 \pm 24.9$ \\
$\begin{array}{l}\text { Overall } \\
\begin{array}{l}\text { Total } \\
\text { (Labeled }+\end{array}\end{array}$ & 162.1 & 110.9 & & & \\
$\begin{array}{l}\text { Non- } \\
\text { labeled) }\end{array}$ & 365.8 & $955.9 \pm$ & $774.9 \pm$ & $1011.7 \pm$ & $1166.5 \pm$ \\
\hline
\end{tabular}

NG, Nodose Ganglion; DRG, Dorsal Root Ganglion; PNS, Parasympathetic Nervous System; SNS, Sympathetic Nervous System 


\section{Table 3}

Cell sampling data between the NG and L6 DRG

\begin{tabular}{ccc}
\hline Sampling Data & NG & L6 DRG \\
\hline Dil+ cells/section (n) & $51.3 \pm 23.3$ & $23.7 \pm 7.2$ \\
\hline Total cells/section $(n)$ & $232.5 \pm 76.7$ & $128.8 \pm 25.9$ \\
\hline Dil+ cells/ganglia (\%) & $21.6 \pm 3.9$ & $18.6 \pm 5.2$ \\
\hline
\end{tabular}

NG, Nodose Ganglion; DRG, Dorsal Root Ganglion 


\section{Retrograde tracer injections}

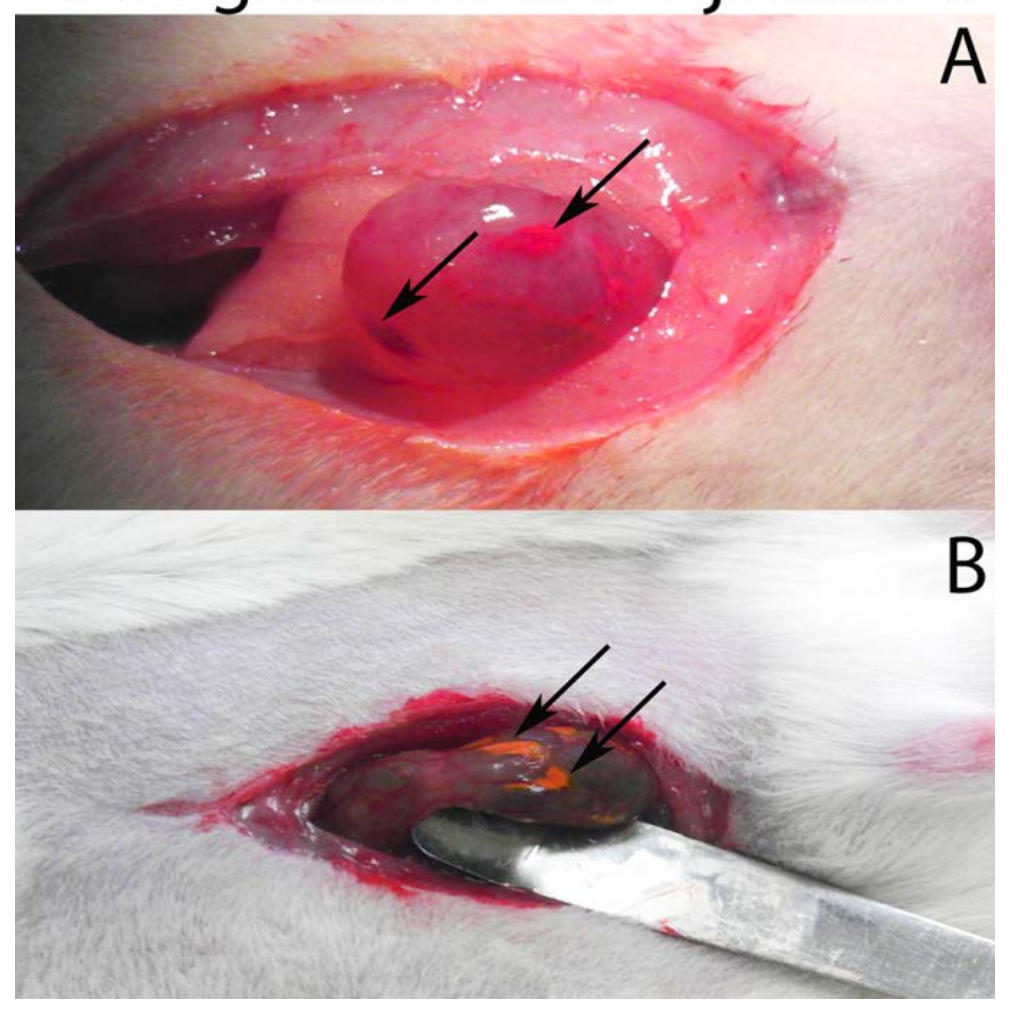

Figure 1 Visceral Organ Tracer Injections

Hamilton microsyringe retrograde tracer injections (arrows) of FAST Dil ${ }^{\mathrm{TM}}$ were made into the wall of the urinary bladder (A) and FAST DiO ${ }^{\mathrm{TM}}$ into the distal colon wall (B). 


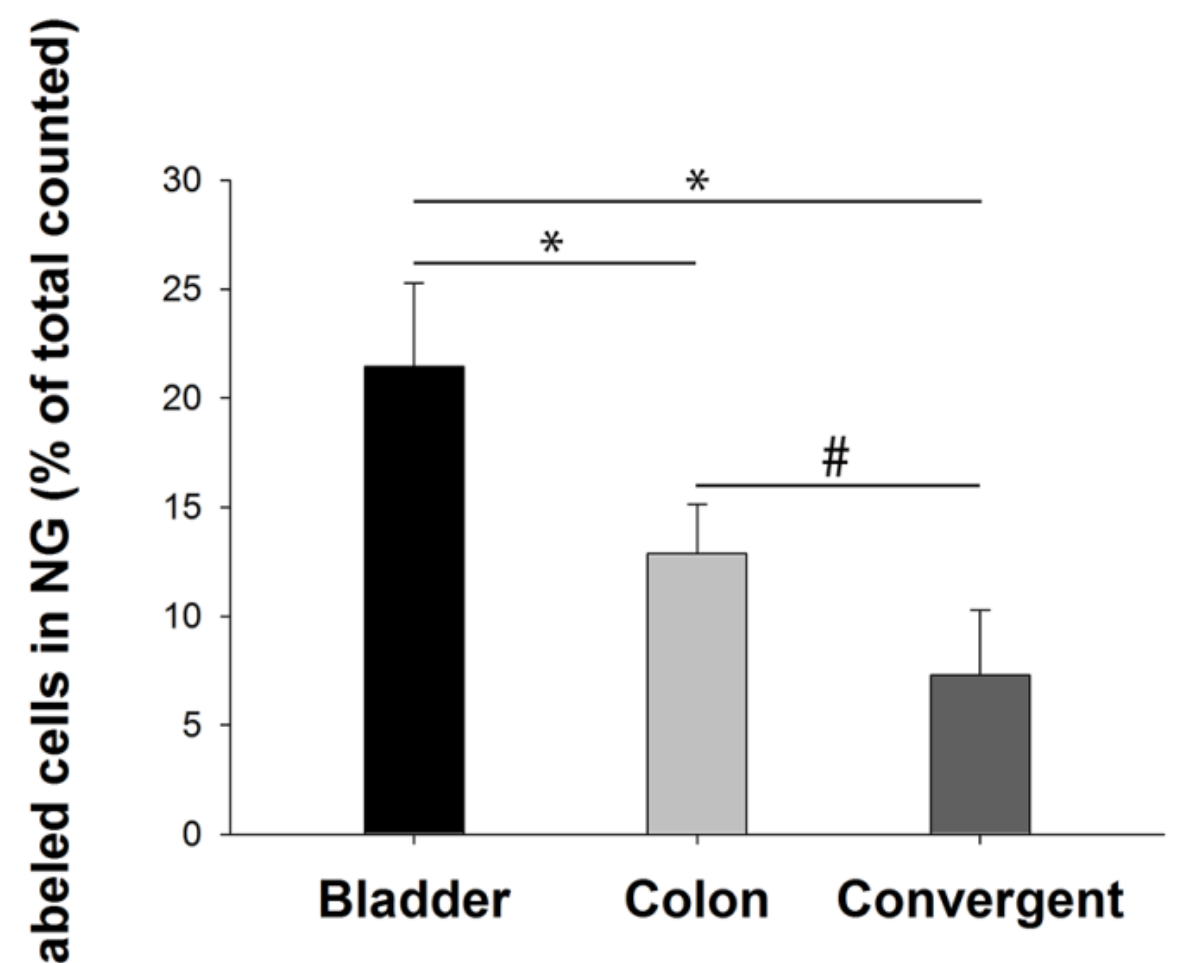

Figure 2 Percentage of bladder-, colon- and convergent-specific neurons in the nodose ganglion (NG)

The percentages are in relation to the total number of neurons counted. There were significantly more bladder labeled neurons than colon $(21.4 \pm 3.1 \%$ vs 12.7 $\left.\pm 1.6 \%,{ }^{*} \mathrm{p}<.001\right)$ and convergent $\left(7.4 \pm 2.8 \%,{ }^{*} \mathrm{p}<.001\right)$ and significantly more colon labeled neurons than convergent (\#p=.001). (Simple bar graph, data are expressed as mean \pm SD, one-way AVOVA with Tukey HSD post hoc t-tests, 15 ganglia total) 


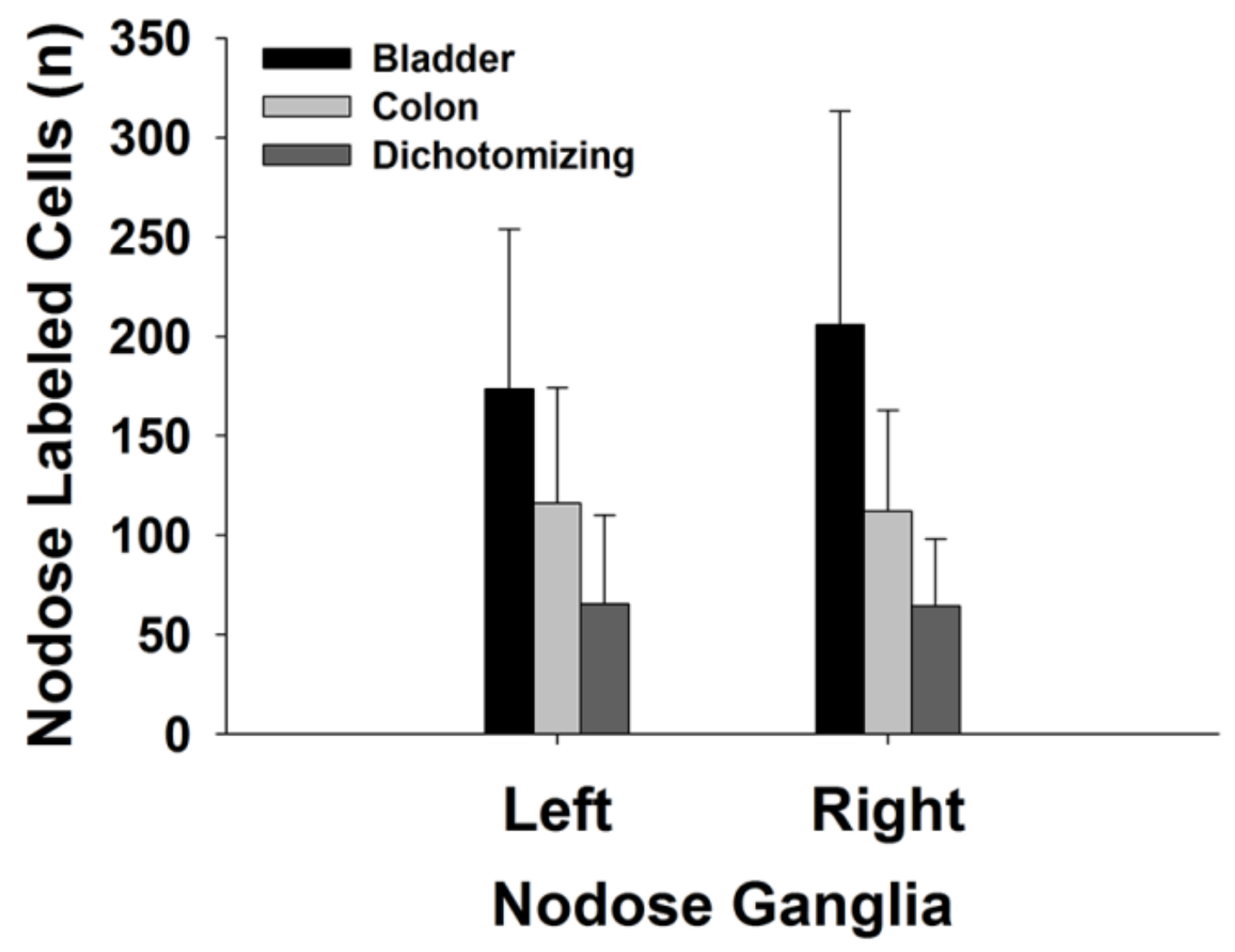

Figure 3 Assessment of side differences between NG

There were no significant differences between the left and right NG using Dil and DiO tracers. (Grouped bar graph, data are expressed as mean \pm SD, part one of RM ANOVA with group factor, 15 ganglia total.) 


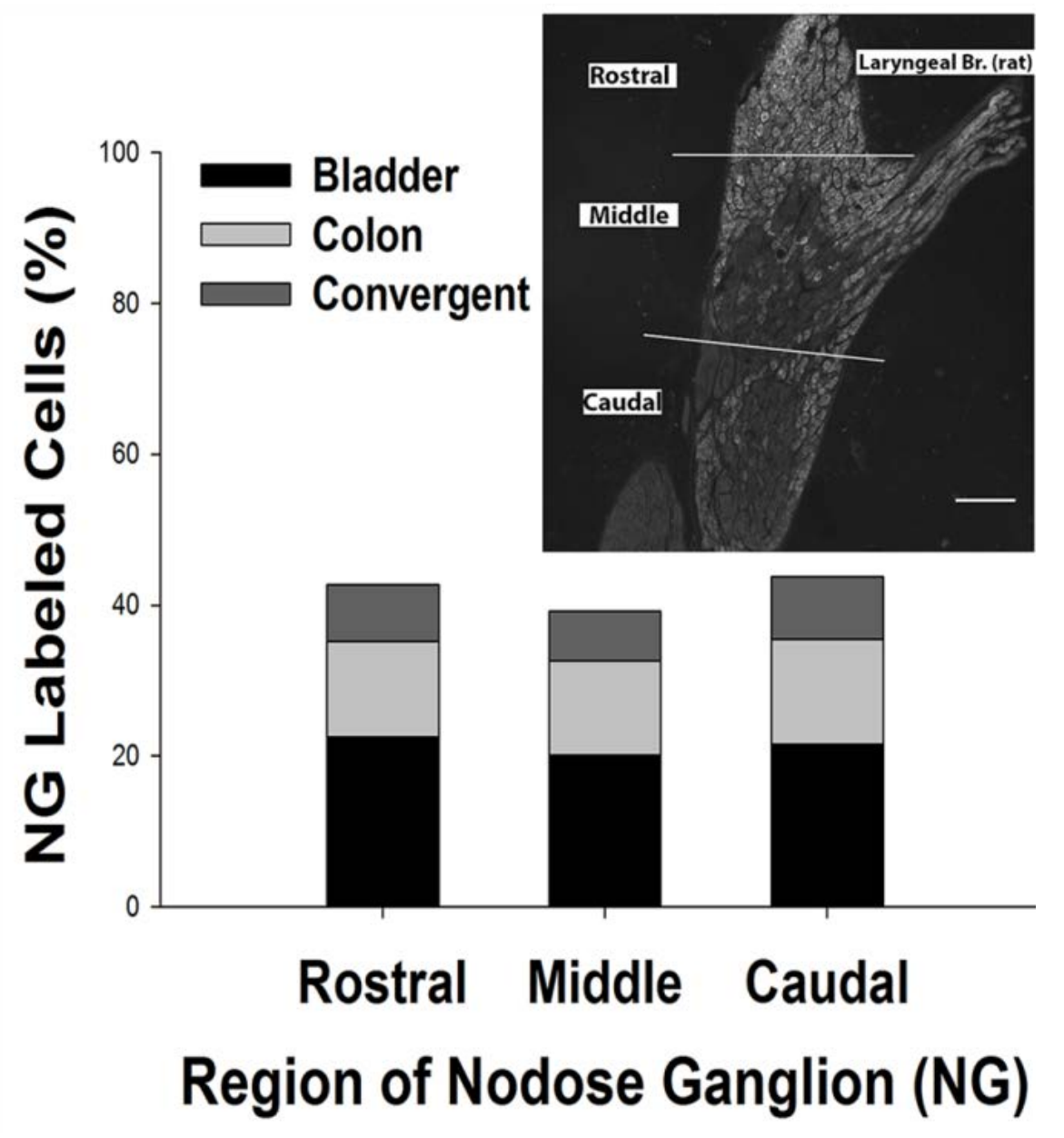

Figure 4 Assessment of regional differences in the NG

No significant differences were found in the percentage of NG labeled cells among rostral (proximal), middle and caudal (distal) regions of the NG using Dil/DiO. Rostral, middle, caudal divisions were demarcated anatomically by the laryngeal branch of the vagus nerve. White scale bar is $200 \mu \mathrm{m}$. (Stacked bar graph, data are expressed as mean \pm SD, part two of RM ANOVA with group factor, 15 ganglia total.) 


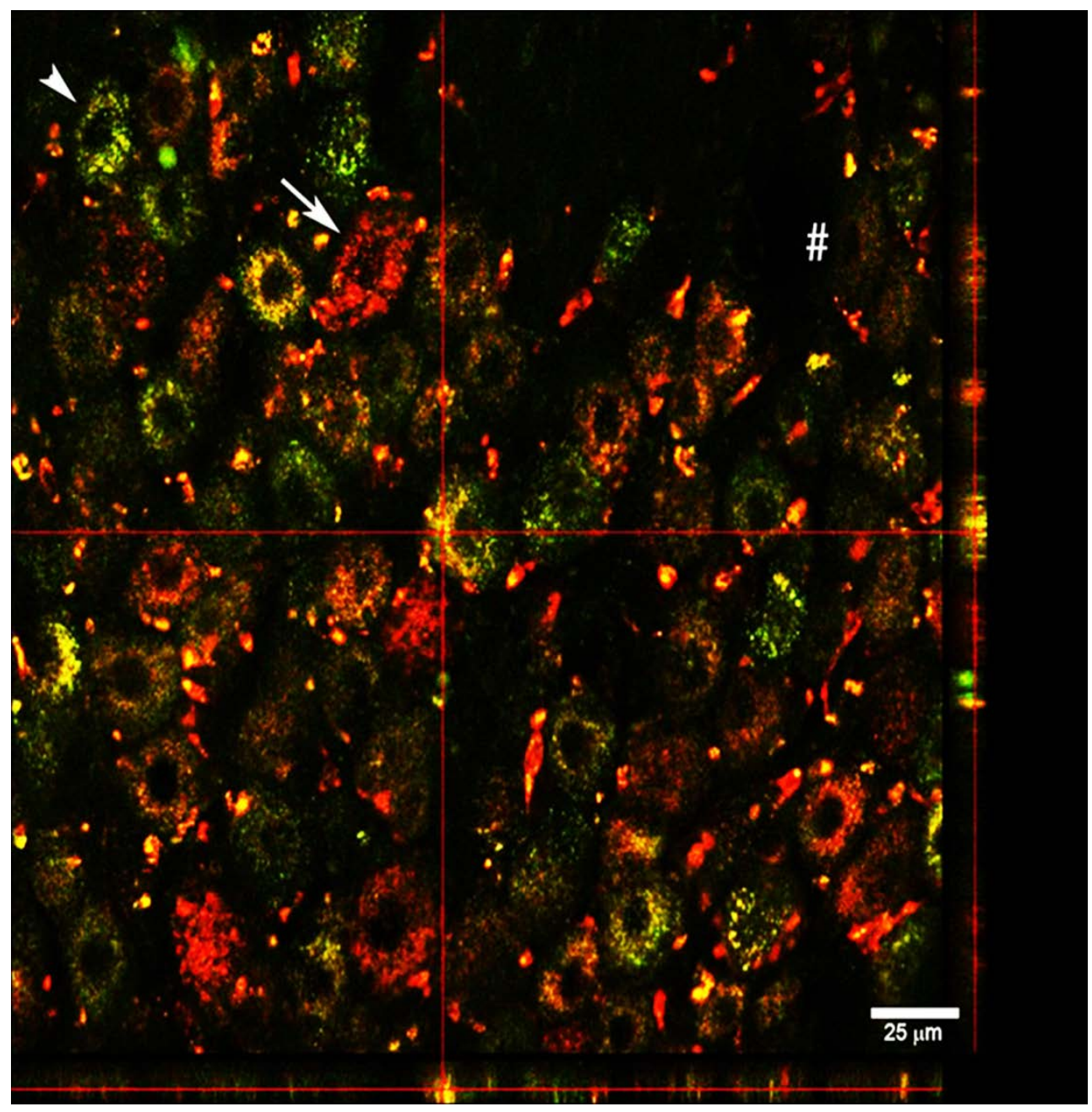

Figure 5 Fluorescent labeling in the NG

A representative confocal image of the NG displayed in the orthogonal view is shown. A bladder-only labeled cell is indicated with an arrow. A colon-only labeled cell is indicated with an arrowhead. A non-labeled cell is indicated with a \# sign. Orthogonal guides (red lines) are centered upon a double-labeled cell (yellow) and the corresponding $X Z$ and $Y Z$ projections are indicated at the bottom and right side of the image, respectively. Only one example of each cell type is indicated above. The scale bar is $25 \mu \mathrm{m}$ (60X objective). 


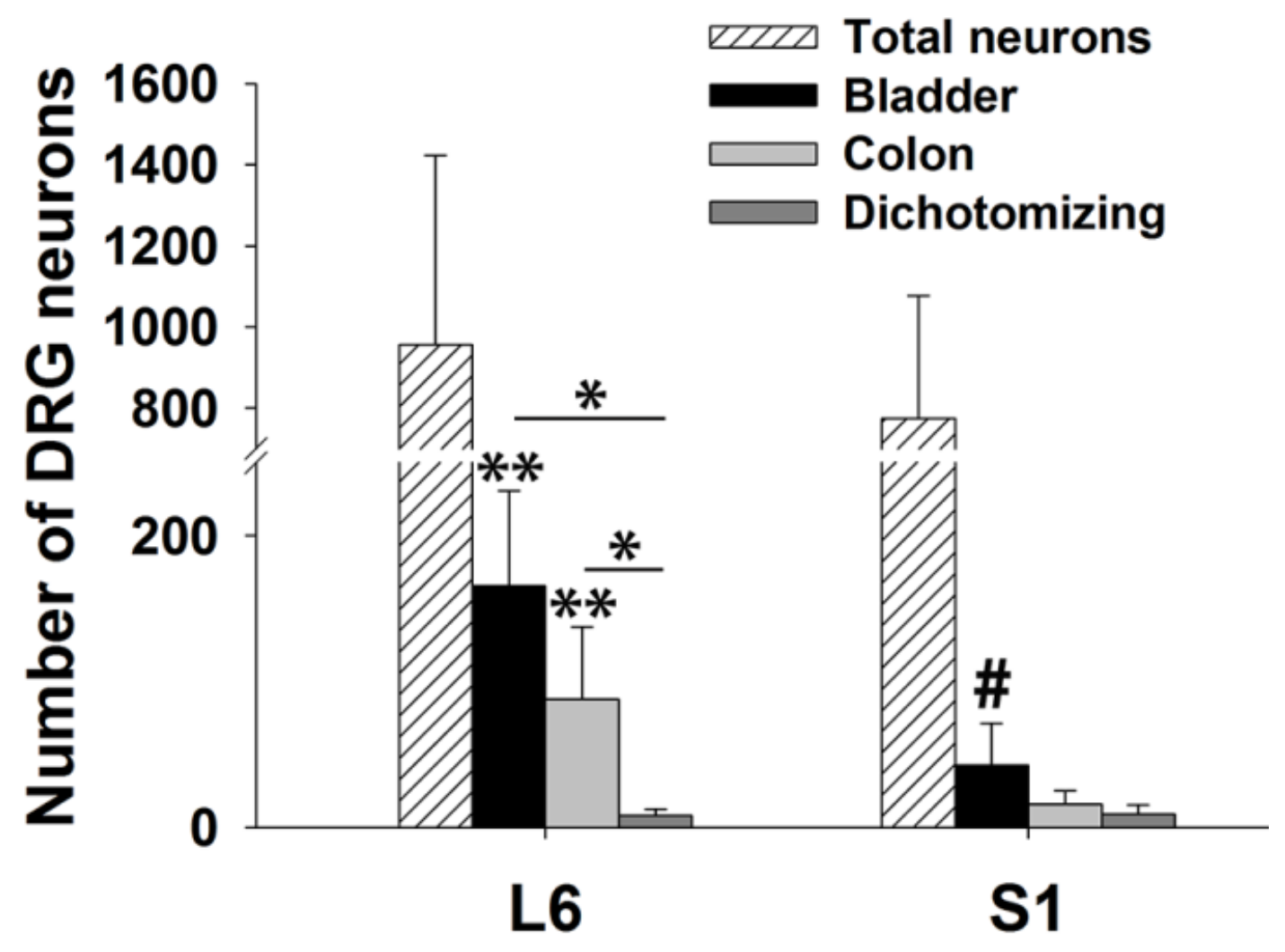

Figure 6 Bladder-, colon- and convergent-specific neurons in the L6 and S1 dorsal root ganglia (DRG)

Within L6, there were significantly more bladder neurons $(165.8 \pm 64.7)$ than colon $\left(87.9 \pm 49.6,{ }^{*} \mathrm{p}<.01\right)$ and convergent $\left(8.1 \pm 4.7,{ }^{*} \mathrm{p}<.01\right)$ neurons and significantly more colon than convergent $\left({ }^{*} p<.01\right)$ neurons. Within $\mathrm{S} 1$ there were significantly more bladder neurons $(42.9 \pm 28.7)$ than convergent neurons $(9.3 \pm$ $6.1, \# p<.05)$. Across the ganglia, there were significantly more bladder and colon labeled neurons in L6 compared to S1 (S1 colon, $15.8 \pm 9.3,{ }^{* *} p<.01$ ). (Grouped bar graph, data are expressed as mean \pm SD, two-way ANOVA with Tukey HSD post hoc t-tests, 10 ganglia (L6) and 14 ganglia (S1).) 


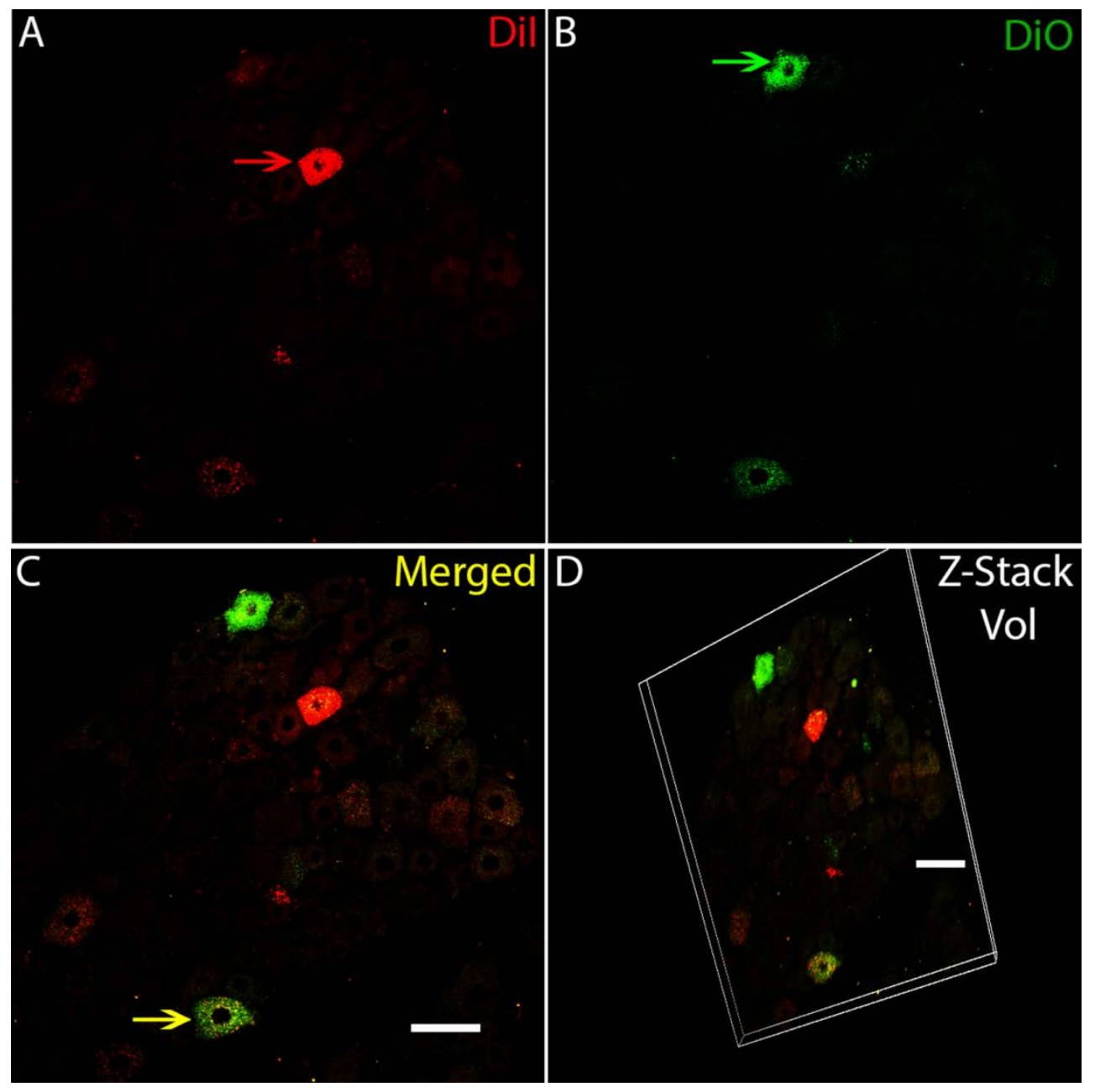

Figure 7 Fluorescent labeling in the S1 DRG

A representative confocal optical section of the S1 DRG is shown revealing in A) a bladder-only labeled cell indicated by the red arrow, in B) a colon-only labeled cell indicated by the green arrow, and in C) a convergent neuron indicated by the yellow arrow. A 3D projection of the cells from A-C is shown in panel D. The

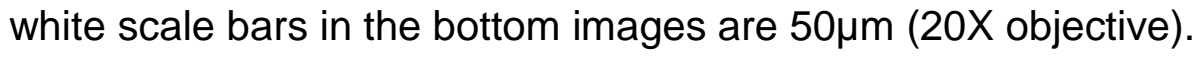




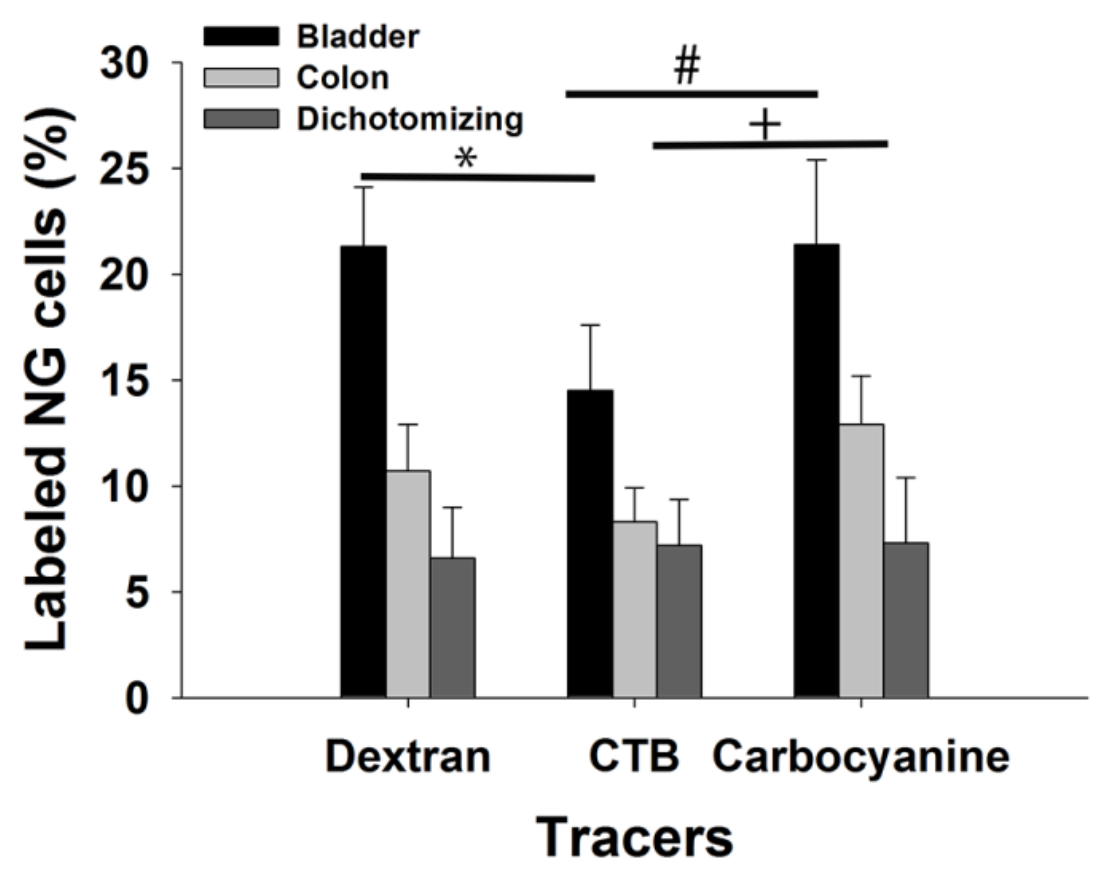

Figure 8 Tracer comparisons in the NG

The percentage of bladder-specific, colon-specific and convergent neurons in the NG after injections of either TMR, CTB-594, or carbocyanine (Dil) into the bladder and FD, CTB-488 or carbocyanine (DiO) into the distal colon showed significantly fewer CTB-bladder and colon labeled cells than Dil (\# bladder, $p<.005$ ), DiO (+ colon, $p=.001$ ) and TMR (* bladder, $p<.05)$ in the NG. No significant differences were found between both dextrans and carbocyanine labeling nor between FD (colon) and CTB-488 (colon) (p>.05). (Grouped bar graph, data are expressed as mean $\pm S D$, one-way ANOVA with Tukey HSD post hoc t-tests, Dextran 2 ganglia, CTB 2 ganglia, Carbocyanine 15 ganglia, TMR=tetramethylrhodamine, $C T B=$ cholera toxin subunit $B$.) 


\section{$\%$ of Labeled Only Neurons}

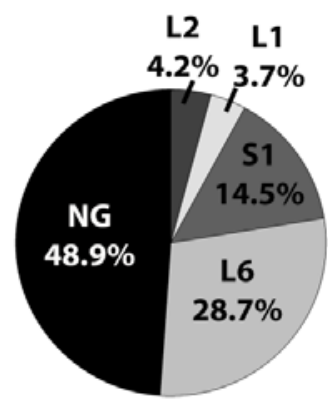

Bladder

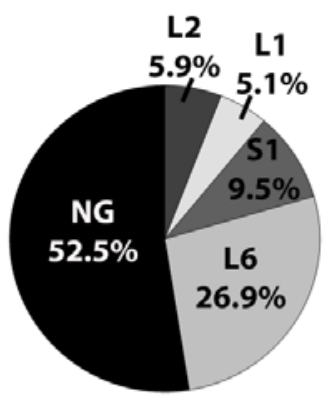

Colon

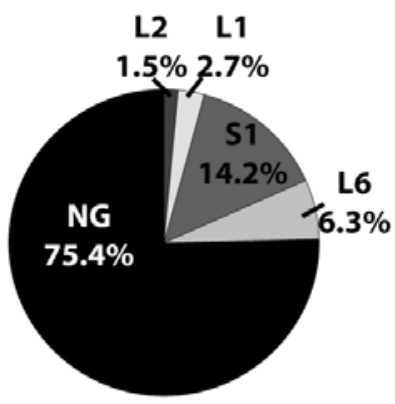

Convergent

$\%$ of Total Neurons (Labeled + Non-labeled)

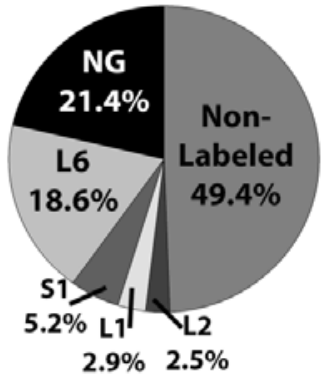

Bladder

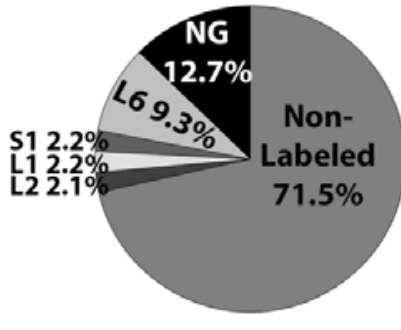

Colon

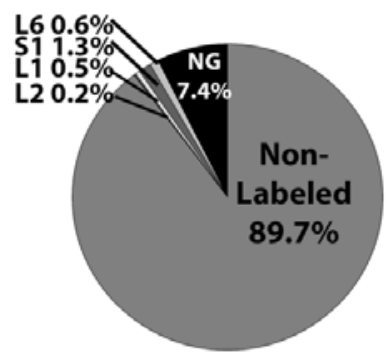

Convergent

Figure 9 Summary diagram of spinal and vagal innervation to the bladder and colon

The top pie graphs represent the percentage of bladder-, colon- and convergentspecific labeling from spinal (L1/L2 and L6/S1) and vagal (NG) sources with respect to only labeled neurons counted. The bottom pie graphs examine the percentage of bladder-, colon-, and convergent-specific labeling from spinal and vagal sources with respect to total neurons counted (labeled and non-labeled). 


\section{CHAPTER III}

THE EFFECT OF SPINAL CORD INJURY ON THE NEUROCHEMICAL PROPERTIES OF VAGAL NEURONS 


\section{$\underline{\text { Introduction }}$}

Spinal cord injury $(\mathrm{SCl})$ results in deficits to sensorimotor systems and profoundly affects the functionality of the autonomic nervous system. Basic research focusing on improving pelvic-visceral outcomes following $\mathrm{SCl}$ is of great clinical importance, since complications such as bladder, bowel and sexual dysfunction affect health and quality of life for this population (Anderson, 2004, Ditunno et al., 2008, Hammell, 2010). Despite the direct immediate impact of injury to the spinal-derived autonomic supply of the pelvic viscera, most of the body's visceral organs also are supplied by a non-spinal source through the vagus nerve. Since the vagus nerve does not travel directly through the spinal cord, its neurocircuitry is often considered intact following SCl. Nevertheless, there is some degree of indirect involvement of both vagal afferents and efferents. For example, following $\mathrm{SCl}$, subsequent neuroplastic-responsive changes have been extensively described within the dorsal vagal complex controlling gastric function (Holmes, 2012). Gastrointestinal (GI) alterations after human upper-thoracic SCI include conditions such as dysphagia (Wolf and Meiners, 2003), esophagitis (Stinneford et al., 1993), peptic ulcerations (Tanaka et al., 1979, Gore et al., 1981), gastroparesis and overall dysmotility (Rajendran et al., 1992, Stinneford et al., 1993, Segal et al., 1995, De Looze et al., 1998, Kao et al., 1999, Williams et al., 2012). Although the mechanisms of $\mathrm{GI}$ dysfunction in humans after $\mathrm{SCl}$ are not thoroughly understood, experimental 
studies in rats suggest that many of the delays in gastric emptying and transit may in part be attributed to vagally-mediated pathways (Gondim et al., 1998, Gondim et al., 1999, Gondim et al., 2001, Tong and Holmes, 2009, Holmes, 2012). In fact, subdiaphragmatic vagotomy has been shown to prevent much of the SCl-induced GI sequelae (Gondim et al., 2001).

The vagus nerve, with sensory cell bodies primarily located in the nodose ganglia (NG), provides innervation to the thoraco-abdominal structures. Despite the view that the vagus nerve does not innervate viscera caudal to the transverse colon, numerous experimental studies have demonstrated that it also provides sensory innervation to the majority of the pelvic viscera (Burden et al., 1983, Gattone et al., 1986, Jancso and Maggi, 1987, Ortega-Villalobos et al., 1990, Altschuler et al., 1993, Hubscher and Berkley, 1995, Collins et al., 1999, Vizzard et al., 2000, Herrity et al., 2014). Even though the vagus nerve has such widespread projections, $\mathrm{SCl}$ does not disconnect the anatomical relationship the nerve has with the tissue it innervates. However, SCl does lead to pathological changes and dysfunction of below-level target organs, such as the bladder (de Groat et al., 1990, Kruse and de Groat, 1993, de Groat et al., 1997), and can thereby influence neuronal phenotype (Vizzard, 1997, Yoshimura et al., 1998, Vizzard, 1999, Yoshimura, 1999, Vizzard, 2000a, Qiao and Vizzard, 2002, Zvarova et al., 2004, Qiao and Vizzard, 2005, Zvarova et al., 2005, Zvara et al., 2006, Ward et al., 2014). Furthermore, various classes of primary sensory neurons, including vagal afferents, have been shown to alter their phenotype and 
the expression of different receptors in response to nerve injury and tissue inflammation (Murphy et al., 1995, Neumann et al., 1996, Li et al., 1999, Michael and Priestley, 1999, Zhou et al., 1999, Banerjee et al., 2009a, Banerjee et al., 2009b, Hill et al., 2010, Wang et al., 2011). In this study, we examined the effects of $\mathrm{SCl}$ on the neurochemistry of the NG.

In this experiment, $\mathrm{P} 2 \mathrm{X} 3$ receptor and SP immunoreactivity (ir) as well as IB4 binding was examined in NG neurons, including those traced from the bladder. These particular markers were selected based on their presence in the NG (Lewis et al., 1995, Michael and Priestley, 1999, Hubscher et al., 2001, Dang et al., 2005, Wang et al., 2014), involvement in the spinal and vagal circuitry (Bradbury et al., 1998, Cockayne et al., 2000, Vizzard, 2001, Young et al., 2008, Zhong et al., 2008, Banerjee et al., 2009a, Mcllwrath et al., 2009) and the potential physiological role these markers may play in nociceptive signaling (Burnstock, 1996, Cook et al., 1997). In addition, anatomical evidence that the vagus nerve provides sensory innervation to the bladder in male rats (Herrity et al., 2014) and the presence of these cellular markers in bladder tissue (Liu et al., 2009, Birder, 2010, Arms and Vizzard, 2011, Birder, 2011, Birder et al., 2012, Birder and Andersson, 2013) adds to the importance of understanding the relationship between target-organ tissue and its innervating neurons. The neurochemical changes that occur in primary afferent neurons may reflect the outcomes of peripheral events. While it is noted that other markers such as TRPV1 (capsacin receptor) and CGRP (calcitonin gene-related peptide) may be 
important to examine in the context of vagal afferent neurochemistry given their presence in dichotomizing vagal afferents (Zhong et al., 2008) and their role in classifying subpopulations of small sensory neurons involved in the sensation of pain (Lawson et al., 2002, Christianson et al., 2006), we felt that examining the selected markers in this study would help us compare and build upon our previous work in both the NG and DRG (Petruska et al., 2000a, Petruska et al., 2000b, Petruska et al., 2000c, Hubscher et al., 2001, Petruska et al., 2002). Additionally, we are limited by the number of microscope optical channels available to image multi-fluorescence immunohistochemistry. We therefore hypothesized that following $\mathrm{SCl}$, the expression profile of known injury-responsive factors $\mathrm{P} 2 \mathrm{X} 3$, IB4 and SP, would be altered in the NG and that the same would be true for NG neurons innervating the bladder. 


\section{Materials and Methods}

\section{Animals}

All experimental procedures were carried out according to NIH guidelines and protocols reviewed and approved by the Institutional Animal Care and Use Committee at the University Of Louisville School Of Medicine. All adult male Wistar rats ( $\mathrm{n}=16$, Harlan Sprague Dawley, Inc, Indianapolis, IN), approximately 250 grams in weight, were individually housed in an animal room with a 12-hour light and dark cycle. They had ad libitum access to water and food (Laboratory Rodent Diet). Groups were either naïve $(n=8)$ or spinal cord injured $(n=8)$. Each group had a subset ( $\mathrm{n}=4$ each) which received retrograde neural tracer injected into the bladder to enable identification of single NG neurons which innervated the bladder.

\section{Spinal cord injury}

Half of the animals $(n=8)$ were anesthetized with a mixture of ketamine (80mg/kg) and xylazine (10mg/kg), injected intraperitoneally, for spinal transection. All surgeries were performed under aseptic conditions and the body temperature was maintained within the range of $36-37^{\circ} \mathrm{C}$ via a warm water recirculator (Gaymar T/Pump, Gaymar Industries Inc, Orchard Park, NY) throughout the surgery and recovery period. Following our previously published protocol (Kaddumi and Hubscher, 2007), a dorsal longitudinal incision was made 
to expose the T7 vertebra and a laminectomy was performed in order to expose the underlying T8 spinal cord. The overlying dura was reflected laterally and the spinal cord cut using a pair of surgical microdissecting scissors. Gentle suction with an air vacuum was used to carefully elevate the cut stump in order to verify the completion of the lesion. Gelfoam (Pharmacia \& Upjohn Company, Kalamazoo, MI) soaked in topical hemostat solution (Henry Schein Inc., Melville, NY) was placed in the lesion cavity. The incision was closed using 4-0 nylon suture for the muscle layers and fascia and surgical clips for the skin. Animals were given subcutaneous injections of ketoprofen (Ketofen, 2.5mg/kg, Fort Dodge Animal Health, Fort Dodge, IA) for analgesia twice a day for 2 days, $0.5 \mathrm{ml}$ of dual penicillin (Penicillin G coupled with Procaine, PenJect ${ }^{\circledR}$, The Butler Company, Columbus, $\mathrm{OH}$ ) in a single dose peri-operatively as a general prophylactic and 5mg/kg gentamicin (GentaFuse ${ }^{\circledR}$, Butler Schein, Dublin, OH) once per day for 5 days to prevent bladder infections. After surgery each animal was housed individually. The urinary bladder was expressed every 8 hours until the micturition reflex occurred automatically, typically 6-12 days after surgery (Hubscher and Johnson, 2000). Animals survived for six weeks followed by euthanasia and tissue removal.

\section{Retrograde tracer injection}

At five weeks post injury, four spinally-transected rats and four agematched naïve control rats were anesthetized with a mixture of ketamine 
$(80 \mathrm{mg} / \mathrm{kg})$ and xylazine $(10 \mathrm{mg} / \mathrm{kg})$. They received a ventral/caudal midline peritoneal incision to expose the urinary bladder which was subsequently manually voided by pressure. Using an established protocol (Rau et al., 2007, Herrity et al., 2014), the fluorescent tracer FAST Dil ${ }^{\mathrm{TM}}$ oil $\left(1,1^{\prime}\right.$-dilinoleyl-3,3,3',3',tetramethylindo-carbocyanine perchlorate, $5 \mathrm{mg}$ dye dissolved in $0.1 \mathrm{ml}$ methanol, Molecular Probes, Inc., Eugene, OR) was injected into the bladder wall with a dye-dedicated 33-gauge needle coupled to a Hamilton microsyringe (Fisher Scientific, Pittsburgh, PA). Note that the abbreviation Dil is used throughout the text/figures/legends to refer to FAST Dil ${ }^{\mathrm{TM}}$ oil. Dye injections were made to the circumference of the trigone, body and dome areas $(10 \mu$ l volume per animal divided into 10 injections of 1ul each; (Herrity et al., 2014)).

Animal body temperature was maintained within the range of $36-37^{\circ} \mathrm{C}$ during surgery via a warm water recirculator (Gaymar T/Pump, Gaymar Industries Inc, Orchard Park, NY). After each injection, the needle was removed slowly and any dye-leakage was removed by cotton-tipped applicators. After injections were completed, the exposed viscera were hydrated as necessary with 5\% Dextrose Lactated Ringers, the abdominal musculature was sutured closed (Ethicon 4-0 non-absorbable surgical suture), the skin closed with Michel clips (Fine Science Tools, Foster City, CA), and a topical antibiotic (Bacitracin, Actavis Mid Atlantic LLC, Lincolnton, NC) applied. Following surgery, animals were placed on a heating pad and core temperature monitored. Post-operative medication was provided as per spinal transection surgery. All animals were 
monitored daily to inspect the surgical incision and identify any changes in an animal's general condition.

\section{Perfusion and Tissue Collection}

All 16 animals were deeply anesthetized with a ketamine $(80 \mathrm{mg} / \mathrm{kg}$ body weight)/xylazine $(10 \mathrm{mg} / \mathrm{kg})$ mixture and transcardially exsanguinated with heparinized saline, followed by $4 \%$ paraformaldehyde perfusion. Each vagus nerve was identified adjacent to the carotid artery and gently separated from surrounding tissues and traced rostrally to the NG, which was excised. Superior cervical ganglia were identified on both sides at the bifurcation of the common carotid artery and removed to be used as control tissue. For the transected group of rats, following a dorsal spinal incision, removal of the spinal cord tissue $1 \mathrm{~mm}$ above and below the transection site was performed. All tissues were placed in individually-labeled tubes of $4 \%$ paraformaldehyde for at least $48 \mathrm{~h}$, followed by immersion in a cryoprotectant solution of $30 \%$ sucrose/phosphate buffer solution with $1 \%$ sodium azide for at least $24 \mathrm{~h}$. Following removal from the cryoprotectant solution, NG's were embedded in OCT ${ }^{\circledR}$ compound (Baxter Scientific) and 12$14 \mu \mathrm{m}$ sections were cut on a cryostat (Leica CM 1850). During retrieval of the bladder, the abdominal cavity and surrounding viscera were inspected for tracer leakage. Overall, gross inspection of all bladder tissue from the $\mathrm{SCl}$ animals did not display evidence of chronic cystitis nor was hematuria or pyuria evident two weeks after surgery (reported time frame for these sequale to surface) which 
could potentially influence the neurochemical properties of NG neurons post-SCI (Hong and Henderson, 1989).

Histology of the lesion epicenter

The lesion cavity, coated in embedding media, was cut into $18 \mu \mathrm{m}$ sagittal sections using a Leica CM 1850 cryostat and mounted onto gelatin-coated histological slides (Azer Scientific, Morgantown, PA). The slides were then stained with both Luxol fast blue and cresyl violet (Kluver-Barrera method) to observe myelin and Nissl substance, respectively. Spot Advanced software (Diagnostic Instruments, Sterling Heights, MI) and the Nikon E400 microscope were used to image the lesion cavity and verify the completeness of the spinal transection (Kaddumi and Hubscher, 2007).

Hindlimb assessment for lesion completeness

We used the Basso-Beattie-Bresnahan (BBB) scale (Basso et al., 1995), an open-field locomotor assessment that is often used to evaluate hindlimb function as an assessment of post-injury spinal cord function. Each animal was placed in an open-field and tested for 4 minutes by the same two scorers, who were presented with injured and non-injured animals in random order. A 21-point scale was used to assess hindlimb coordination and rated parameters such as individual joint movements (0-7), weight support (8-13) and paw placement (1421). Intact animals should demonstrate a locomotor score of 21; whereas 
animals that receive a completeT8 transection should exhibit complete paralysis of the hindlimbs (0 score). However, due to the fact that the lumbar enlargement is intact and receives afferent input from the hindlimbs, BBB scores following complete transection are typically higher than 0 , averaging around a score of 3 (extensive movement of 2 joints) (Basso et al., 1996, Singh et al., 2011). Note that to prevent any functional connections across the lesion site from potential spared tissue, gelfoam was placed between the two cut stumps. It has been demonstrated that as little as $4-5 \%$ sparing (primarily in the ventrolateral funiculi) was sufficient for attaining a BBB score of 7 following "complete" spinal transection (no gelfoam used across lesion) (Fang et al., 2011). A combination of the BBB score along with an assessment of sparing at the lesion cavity was used to determine the degree of "completeness" following $\mathrm{SCl}$ in this study. Note that one animal from the transection group was removed in this study due to a BBB score of 6 and an incomplete lesion.

Immunofluorescence histochemistry

Sections were thaw-mounted onto slides and allowed to air-dry. They were then encircled with hydrophobic resin (PAP Pen, Research Products International Corp). Slide-mounted sections were incubated at room temperature for $2 \mathrm{~h}$ in a solution of $2 \%$ Triton $\mathrm{X}-100 \AA$ in phosphate buffered saline (PBS). This pre-treatment step improves the quality of P2X3-ir (Petruska et al., 2000a). The slides were rinsed in distilled water and then incubated for $30 \mathrm{~min}$ in a solution of 
10\% normal donkey serum (Jackson Immuno Research, West Grove, PA) in PBS with $0.3 \%$ Triton X-100 (MP Biomedicals, LLC, Solon, OH) to block nonspecific antibody binding. The immunohistochemical reagents and the labeling procedures are summarized in Table 4. Incubations in primary antisera were performed overnight (14-18 hours). All steps were followed by multiple rinses with PBS. All fluorescent secondary antisera were diluted 1:100 and incubations were 2 hours. The tyramide signal-amplification (TSA ${ }^{\mathrm{TM}}$ ) reagent kit (SigmaAldrich, St. Louis, MO) (Bobrow et al., 1989, Bobrow et al., 1991, Bobrow et al., 1992) was used at 1:100 and incubations were for 4-5min (Rau et al., 2007). Once all steps were completed, the slides were coverslipped with a glycerolbased photobleach-protective medium (Fluoromount-G, Southern Biotech).

\section{Cell Quantification}

To view labeled sections, imaging was performed using the Nikon Eclipse TiE inverted microscope with NIS Elements software. Initially, images were captured using a 10x lens (APO DIC N1 10x/0.45 NA, Nikon) with consistent exposure times and computationally stitched together to visualize whole ganglion sections. Imaging of individual fluorophores was achieved with a mercury-arc lightsource and the following filter sets: for Cy3 [543/22nm excitation, 593/40nm emission, 562nm dichroic, Semrock]; for Alexa Fluor 488 [470/40nm excitation, 525/50nm emission, 495nm dichroic, Nikon]; for Alexa Fluor 350 [350/50nm 
excitation, 460/50nm emission, 400nm dichroic, Nikon]; for Cy5 [615/70nm excitation, 700/74nm emission, 660nm dichroic, Nikon].

In order to obtain unbiased percentages of $\mathrm{P} 2 \mathrm{X} 3, \mathrm{SP}$ and IB4 positive neurons in the NG, the physical dissector method was applied (Pakkenberg and Gundersen, 1988, Coggeshall and Lekan, 1996). Across the entire ganglion, assembled by automated stitching, counts of all singly-, multi- as well as nonlabeled/other (collectively comprising total neuronal counts) NG neurons were made by a scorer blinded to treatment groups. Starting with a random section, neurons with a clearly visible nucleus and definable soma were counted only if they were not present in an adjacent "look up" serial section. As an added measure to avoid double counting single neurons, the counting-sections were at least 60 microns apart (every $5^{\text {th }}$ section). To differentiate background from foreground pixels, threshold values also were obtained based off the image histogram for each marker and held constant for each image quantified. Note that non-labeled/other cells ( $\mathrm{NeuN}+$ only) were quantified and represented the total neuron population (Mullen et al., 1992, Gittins and Harrison, 2004, HerculanoHouzel and Lent, 2005). Images of tissue without application of the primary antibody were taken and utilized as baseline controls (images not shown).

Positive neuron counts were expressed as a percentage of the total number of neurons (NeuN+ only) from within the entire stitched ganglion as well as a percentage of all labeled neurons. An Olympus 3 Laser scanning confocal microscope with Fluoview 500 software (Mellville, NY) and a Nikon A1R MP+ 
confocal microscope with Elements software were used to collect high resolution, serial optical sections of NG neurons.

\section{Statistics}

Analyses were performed using SPSS v19-20 (IBM, North Castle, NY). Levene's statistic was applied for homogeneity of variances and data are expressed as mean \pm standard deviation (SD). A one-way analysis of variance (ANOVA) with Tukey HSD post hoc t-tests was performed for the assessment of all histochemical markers and NG bladder labeling within groups. For the analysis of the histochemical markers between groups, data were normalized as percentages of total NG cells and analyzed via a two-way ANOVA with Tukey HSD post hoc t-tests. For all other analyses, two-tailed Student's t-tests were performed assuming equal variance. Statistical significance was defined as $p \leq .05$. 


\section{$\underline{\text { Results }}$}

Immunohistochemical signature of NG neurons

IB4 binding in NG neurons was localized to the plasma and axonal membranes as well as the Golgi complex. Immunoreactivity (-ir) for P2X3 and SP was present in the cytoplasm and P2X3-ir also could be found in the plasma and axonal membranes. All of the patterns of staining for these markers were consistent with previous studies in both NG and DRG (Averill et al., 1995, Petruska et al., 2000a, Hubscher et al., 2001, Vulchanova et al., 2001, Banerjee et al., 2007). When examining the total percentage of labeled NG neurons, all of the markers were well represented (Figure $10 \mathrm{~A}$ ), with the majority of NG neurons binding IB4 (IB4, $65.5 \pm 6.2 \%$ versus SP, $31.1 \pm 10.3 \%$; IB4 versus P2X3, $51.2 \pm 7.9 \%$ ). Overall, when considering all possible combinations of the markers used, there were 8 different histochemical signatures represented in the NG (Figure $10 \mathrm{~B})$. The most prevalent combinations were neurons that were IB4+ only followed by the P2X3+ only and IB4+/P2X3+/SP- combinations (IB4+ only versus $\mathrm{P} 2 \mathrm{X} 3+$ only, $33.3 \pm 7.1 \%$ versus $15.0 \pm 3.3 \%$, $\mathrm{p}<.001$; IB $4+$ only versus IB4+/P2X3+ only, $33.3 \pm 7.1 \%$ versus $15.0 \pm 5.0 \%, p<.001)$. A typical example of the quadruple immunohistochemical staining in the NG is provided in Figure 11. 
Effect of SCI on NG neurons expressing P2X3, SP and binding IB4

Following a chronic transection injury at T8, BBB assessments at the 6 week time point for all transected animals revealed an average score of $(0.9 \pm$ 0.9). Examination of the cellular markers after $\mathrm{SCl}$ revealed a significant increase in the percentage of NG neurons expressing P2X3 $(p<.001)$ as well as a significant decrease in the percentage of NG neurons binding IB4 $(p<.05)$ relative to non-injured controls (Figure 12). There were no significant differences in SP or NeuN expression between groups. P2X3 expression and IB4 binding in the NG are demonstrated in Figure 13. Within the transected group, the percentage of neurons expressing P2X3 and the percentage of neurons binding IB4 were each significantly greater than the percentage of neurons expressing SP, which was unchanged from the non-injured group of animals (P2X3, $27.3 \pm 4.8 \%$ versus SP, $6.5 \pm 2.8 \%, p<.01 ;$ IB $4,23.7 \pm 6.5 \%$ versus SP, $6.5 \pm 2.8 \%, p<.01 ; S P, 6.5 \pm$ $2.8 \%$ versus SP-non-injured, $5.8 \pm 3.8 \%$ ). With respect to the total number of labeled neurons within their individual populations (i.e. all P2X3+ neurons and all IB4+ neurons), the distribution of the P2X3+/IB4-/SP-only subset represents $50.7 \%$ of all P2X3+ neurons, while the IB4+/P2X3-/SP-only subset represents $42.6 \%$ of all IB4+ neurons. Overall, these populations of neurons comprised about half of the total population of NG neurons examined. 
Immunohistochemical profile of bladder non-injured and injured NG neurons

The retrograde tracer Dil, was injected into the bladder wall in order to determine if the subsets of NG neurons affected by spinal cord transection include those that supply the bladder. Initially, we found that the percentage of NG neurons traced from the bladder in both groups in this study is similar to our previous study of spinally intact animals $(22.2 \pm 3.6 \%$ versus $21.4 \pm 4.0 \%$, (Herrity et al., 2014)) .

With respect to the total population of $\mathrm{P} 2 \mathrm{X} 3+\mathrm{NG}$ neurons after chronic $\mathrm{SCl}(50.7 \pm 8.2 \%)$, bladder-innervating neurons (Dil+/P2X3+//B4-) represented $32.8 \pm 1.1 \%$, while with respect to the total population of neurons that were IB4+ after injury (42.6 $\pm 5.1 \%)$, bladder-innervating neurons (Dil+/P2X3-/IB4+) represented $21.5 \pm 7.4 \%$. Overall, in these two distinct subsets of NG neurons, more than half of the neurons are traced from the bladder (Figure 14). Images of the Dil+/P2X3+ and Dil+/IB4+ subsets following transection are demonstrated in Figure 15. Note that in this study, the proportion of NG neurons traced from the bladder in spinally-intact rats $(23.7 \pm 3.6 \%)$ did not differ significantly from the proportion traced from the bladder after chronic spinal transection injury $(20.2 \pm$ $3.0 \%)$. 


\section{Discussion}

Visceral organs including those in the pelvic region have a dual sensory innervation from spinal and non-spinal (i.e., the vagus nerve) sources (Burden et al., 1983, Gattone et al., 1986, Jancso and Maggi, 1987, Ortega-Villalobos et al., 1990, Hubscher and Berkley, 1995, Collins et al., 1999, Herrity et al., 2014). This study, using immunohistochemical techniques, examined the vagal component by assessing changes in the presence of P2X3, IB4 and SP in NG neurons following $\mathrm{SCl}$. Following spinal transection at $\mathrm{T} 8$, we evaluated potential plasticity in subsets of NG neurons which contain projections that bypass the spinal cord from visceral organs, including those projections that specifically supply the bladder. A major traumatic event to the nervous system, such as $\mathrm{SCl}$, leads to dysfunction in multiple organ-systems, and ultimately influences the neurons that innervate these tissues. In this study, vagal sensory cell bodies displayed an increase in P2X3 expression and a decrease in IB4 binding, which also held true for many neurons innervating the bladder. These results suggest that NG neurons, including the bladder subset, are sensitive to a spinal injury and are capable of responding by modifying their phenotype.

Immunohistochemical phenotype of NG neurons

Overall, from the cellular markers examined in this study, the majority of NG neurons were IB4+. Isolectin B4 has been shown to label primarily a subpopulation of non-peptidergic spinal sensory afferents that respond to 
noxious stimuli and are thought to be functionally distinct from peptidergic or neurons that are IB4 negative (Stucky and Lewin, 1999). Even though IB4+ neurons, in general, are widely expressed within the NG (Silverman and Kruger, 1990, Li et al., 1997, Hubscher et al., 2001, Young et al., 2008, Zhong et al., 2008), the function of this population in vagal afferents is still unclear. For instance, vagal afferents are largely known for their involvement in conveying information about the physiologic state of the viscera to the brain as part of homeostatic regulation (Cervero, 1994, Janig, 1996, Grundy, 2002). In the gastrointestinal tract, vagal afferent fibers are responsive to stretch and tension as well as to locally released hormones following the ingestion of food (Berthoud and Powley, 1992, Wang et al., 1997, Phillips and Powley, 2000, Berthoud et al., 2001). Although they are typically not responders to visceral stimuli within the noxious range (Ozaki et al., 1999), previous data from our lab and that of others suggest otherwise. For example, while spinal afferents may be responsible for relaying mechanical nociceptive information, vagal afferents may play more of a predominant role in conveying chemical nociceptive stimuli, thus contributing to disease-related conditions stemming from visceral hyperalgesia (Schuligoi et al., 1998, Michl et al., 2001, Page et al., 2002, Holzer, 2003, Lamb et al., 2003, Danzer et al., 2004, Holzer et al., 2004, Sugiura et al., 2005, Kaddumi and Hubscher, 2007). Therefore, besides the known role of vagal afferents in relaying homeostatic information to the brain, the population of vagal afferents that also are IB4+ may be involved in visceral nociceptive processing (based on the 
putative role of the majority of DRG IB4 binding neurons (Vulchanova et al., 2001, Petruska et al., 2002)).

In the current study, it was shown that many vagal visceral afferents innervating the bladder also were IB4+. In other NG visceral afferents and in line with the results here, labeled vagal afferents from the stomach and duodenum demonstrated a substantial percentage of IB4 binding (Zhong et al., 2008). Furthermore, numerous studies identify a low percentage of calcitonin gene related peptide (CGRP)-ir or peptide-containing NG neurons projecting to thoraco-abdominal viscera (Green and Dockray, 1987, Zhuo et al., 1997, Dutsch et al., 1998, Ichikawa and Helke, 1999, Wank and Neuhuber, 2001, Hoover et al., 2008). Despite the fact that a large proportion of visceral NG neurons appear to be IB4+, the functional significance of these specific subsets also has yet to be determined, as evidence of particular markers for the coding of vagal afferent subtypes are limited (Berthoud and Neuhuber, 2000). One exception may be calretinin (calcium binding protein), which appears to be expressed specifically by cervical esophageal vagal afferents (Dutsch et al., 1998).

Similar to other studies reporting P2X3 receptor expression in vagal sensory cell bodies using immunohistochemical techniques we also found that P2X3 receptors were highly prevalent and distributed throughout the NG (Banerjee et al., 2009a, Wang et al., 2014). These findings suggest that a large population of vagal afferents are sensitive to ATP and thus through purinergic signaling mechanisms, serve an important role in mechanosensory transduction. 
When considering individual subsets of neurons based on the cellular markers examined in this study, P2X3 receptor expression was present in about half of the IB4+ NG neurons, a co-expression subset we have reported previously in the NG (Hubscher et al., 2001) and others in the DRG (Vulchanova et al., 1996, Vulchanova et al., 1997, Bradbury et al., 1998, Vulchanova et al., 1998, Xiang et al., 1998).

In regards to the overall population of NG neurons, the percentage of SP+ only neurons we found was similar to an earlier report, around 30\% (Wu et al., 2005). In general, SP may be an important neuropeptide utilized in vagal afferent synaptic transmission. While it is noted that SP+ neurons are abundant in the NG (Zhuo et al., 1997), their distribution has been reported to be located near the rostral pole of the ganglion (Helke and Niederer, 1990, Zhuo et al., 1997, Wu et al., 2005). We and others have previously found a homogenous distribution of visceral labeling throughout the NG (Sharkey and Williams, 1983, Altschuler et al., 1989, Zhong et al., 2008, Herrity et al., 2014). Although we did not assess the existence of an organotypic distribution of labeling for the histological markers of interest within the NG in this study, the presence of the many SP-ir neurons in the rostral region may be anticipated due to the fact that it is anatomically close to the jugular ganglion, which is primarily peptidergic in its neurochemical composition (Undem et al., 2004, Plato et al., 2006). The proximity of neurons with similar neurochemical phenotypes may be important for performing like 
functions and even for sensory afferent integration (Browning and Mendelowitz, 2003).

Effect of SCl on P2X3-ir in NG neurons

Following chronic $\mathrm{SCl}$, two significant changes were observed in subsets of NG neurons. The first finding was a significant increase in the number of neurons expressing P2X3-ir in the spinal-transected group relative to non-injured controls. In the somatosensory system, alterations in P2X3 expression following nerve injury have been mixed. Both down-regulation (Bradbury et al., 1998) and up-regulation (Eriksson et al., 1998, Novakovic et al., 1999) of the receptor have been documented in various peripheral nerve injury models such as axotomy, ligation and chronic constriction. In both studies where there were increases in P2X3 expression, the injury model used resulted in some neurons that would be potentially "uninjured". To assess these differences, activating transcription factor 3 (ATF3), a marker of peripheral nerve injury and absent from intact neurons (Tsujino et al., 2000), identified decreased P2X3 (mRNA) in ATF3-ir neurons, while the increased expression was evident in the intact subset of neurons (Tsuzuki et al., 2001). The significant increase in NG P2X3-ir found in the present study was consistent with the ATF3-ir findings (Tsuzuki et al., 2001), since the vagal afferents are likely not directly injured given they by-pass the $\mathrm{SCl}$, though this has not yet been directly tested. 
Even though contact between the vagus nerve and its peripheral targets has not been severed, there is an overall effect of injury on the vagal afferent neurocircuitry (Hubscher and Berkley, 1995, Kaddumi and Hubscher, 2007) and the increased P2X3 expression seen in the NG here may help to improve our understanding of the indirect effect on the vagal system after injury. For instance, the increased P2X3-ir following SCl may be attributed to an inflammatory reaction of the system due to the nature of the injury itself. Acutely, SCI triggers an inflammatory response characterized by various cellular events such as the synthesis of cytokines, chemokines and the infiltration of leukocytes, neutrophils and monocytes (Bartholdi and Schwab, 1997, Klusman and Schwab, 1997, Popovich et al., 1997, Bethea et al., 1998, Hausmann, 2003, Saville et al., 2004, Fleming et al., 2006, Stirling and Yong, 2008), which, over time, systemically may affect tissues outside the central nervous system leading to organ dysfunction. Released inflammatory cells from the blood stream can impact the functionality of different viscera due to the intimate relationship these organs have with the vascular system (Campbell et al., 2005, Gris et al., 2008, Bao et al., 2011). In addition, both acute and chronic SCl induce significant changes in organs with spinal innervation from segments below the lesion-level. Organs such as the bladder experience substantial stress and histopathology, which can lead to alterations in the integrity of the lining of the bladder (Apodaca et al., 2003) making the bladder more susceptible to chronic inflammation (Herrera et al., 2010). 
Structural changes after $\mathrm{SCl}$ also include bladder (detrusor muscle) hypertrophy, which triggers a release of neurotrophic factors such as NGF from the urothelial lining (Vizzard, 2000a, Seki et al., 2002, Vizzard, 2006, Fowler et al., 2008). Increases in NGF following SCI (Yoshimura, 1999, Vizzard, 2000a, Ward et al., 2014) or inflammation (Oddiah et al., 1998, Steers and Tuttle, 2006) as well as other excitatory neurotransmitters such as ATP (Sun et al., 2001), play a major role in neuro-epithelial interactions. For example, in a migraine headache model, retrograde transport of NGF from the periphery to the trigeminal ganglion or exposure of trigeminal afferents to NGF led to an upregulation of P2X3 receptor protein in the cell bodies (D'Arco et al., 2007, Giniatullin et al., 2008).

Given that the vagus nerve provides a substantial degree of innervation to the bladder (Herrity et al., 2014), the fact that we found many co-labeled Dil+/P2X3+ NG neurons after injury in this study, the presence of the high affinity receptor for NGF (TrkA) (Zhuo and Helke, 1996, Helke et al., 1998, Lamb and Bielefeldt, 2003) and low affinity (p75) receptor (Verge et al., 1992, Zhuo et al., 1997) and that vagal afferents have the capability to transport NGF (Helke et al., 1998), the phenotypic changes with respect to $P 2 X 3$-ir in the bladder-innervating NG neurons have the potential to be mediated through the actions of NGF. Importantly, in a manner distinct from the actions of NGF (D'Arco et al., 2007), CGRP-mediated insertion of P2X3 into the cell-surface membrane is an alternative mechanism, which has been demonstrated in sensitized trigeminal ganglion neurons (Fabbretti et al., 2006). However, since the majority of CGRP 
expressing neurons appear to reside in other cranial ganglia (petrosal, trigeminal, glossopharyngeal and jugular) compared to the NG (Helke and Hill, 1988, Helke and Niederer, 1990, Helke, 2005, Hayakawa et al., 2010) this molecular mechanism may indirectly affect NG neurons, perhaps acting at a distance through en passant synaptic contact (Koerber et al., 1999).

The P2X3 receptor, predominantly expressed on sensory afferents (Chen et al., 1995, Lewis et al., 1995), including vagal fibers (Kestler et al., 2009), also can be separately retrogradely transported from the periphery to the cell body via endosomes (Chen et al., 2012). This retrograde transport is thought be important for maintaining neuronal activity and cell excitability through activation of transcription factors (Chen et al., 2012). In disease states, such as $\mathrm{SCl}$, the extracellular milieu of ATP may be relatively high compared to healthy states, where excesses are rapidly hydrolyzed (North, 2004, Khakh and North, 2006, Burnstock, 2007). Large amounts of ATP (likely released from damaged tissue (Cook and McCleskey, 2002)), can signal through P2X3 receptors and may show that P2X3 has a more extensive role in the NG besides normal visceral afferent transduction, perhaps contributing to nociceptive signaling following injury or tissue inflammation.

\section{Effect of SCl on IB4 binding in NG neurons}

The second change following chronic transection injury was a decrease in NG IB4 binding relative to controls. This finding is similar to that of others in 
cases where decreases in the total number of IB4 binding DRG neurons on the contralateral side (uninjured side) also have been demonstrated following L5 spinal nerve transection (Li and Zhou, 2001). Importantly, the numbers remain reduced at the chronic time point (5 weeks post-injury), suggesting the effect was not transient.

An explanation for the decrease in IB4 binding may be attributed to a stress response to the system following transection. Since glial cell-line derived neurotrophic factor (GDNF) supports and aids in the regulation of IB4 neurons post-natally (Molliver et al., 1997), perhaps some disruption to its availability or receptor complex as well as alteration to the IB4 binding glycoconjugate could explain the observed decrease (Peyronnard et al., 1989, Bennett et al., 1998). However, in response to peripheral nerve injury, spared IB4 neurons also demonstrate the capability to sprout, forming perineuronal nets with both satellite and adjacent cells within the ganglion (Li and Zhou, 2001). It has been suggested that a mechanism behind this spouting in response to nerve injury may involve inflammatory environmental changes that create a chemotactic gradient, attracting various chemokines (Bogen et al., 2009). This communication between injured and non-injured "neighbors" within the ganglion may serve as a basis for cross-excitation and could eventually induce hyperalgesia or allodynia (Amir and Devor, 2000, Bogen et al., 2005). Even though the injury model used in this study does not directly injure vagal neurons, they could be considered "spared" 
neurons that also demonstrate a phenotypic switch in response to CNS damage and have the potential to drive visceral nociceptive signaling.

Effect of SCl on SP expression in NG neurons

SP is one of the main neuropeptides released from a proportion of primary afferent terminal endings that express SP, in response to irritation or inflammation (Avelino et al., 2002, Bueno and Fioramonti, 2002, Domotor et al., 2005) and is present in NG neurons (Zhuo et al., 1997). No significant differences in SP-ir were present in this study between transected and noninjured groups. A previous report assessing changes in NG neurotransmitters found that SP-ir was unaffected by vagal axotomy (Helke and Rabchevsky, 1991). The lack of changes in the NG with respect to SP-ir following injury does not preclude any particular alterations at terminal endings, either peripherally in target organs or centrally (solitary nucleus). For instance, there is a high concentration of SP afferent terminals, primarily of vagal origin, present in solitary nucleus (Helke et al., 1980, Maley and Elde, 1982, Maley et al., 1983, Maley et al., 1987, Maley, 1996, Zhuo et al., 1997). Alternatively, there may be molecular pathway alterations involved in the release of SP and translation at the cell body (Tang et al., 2007, Pan et al., 2010). An acute SCI or direct tissue inflammation model (such as acetic acid instillation into the bladder) may provide more insight to vagal SP expression in the rat (Banerjee et al., 2007, Pan et al., 2010). 
Alterations to bladder NG neurons following SCI

$\mathrm{SCl}$ did not result in differences compared to non-injured controls in the number of NG neurons labeled from bladder, confirming these vagal afferents remain intact after cord transection. Although vagal afferents exhibit a high degree of neurochemical and electrophysiological plasticity in response to trauma and inflammation (Zhang et al., 1996, Myers et al., 2002, Browning and Mendelowitz, 2003, Peters et al., 2013), it is likely that the observed neurochemical changes in this study are a result of interactions with the target organs these vagal fibers innervate rather than direct neural damage. It should be noted, however, that other, extrinsic sources of ATP can reach P2X3 receptors through release from sympathetic neurons, tumor cells or from vascular endothelial cells associated with ischemia (Burnstock, 1996).

In the two subsets of NG neurons that changed after $\mathrm{SCl}(\mathrm{P} 2 \times 3+$ only and IB4+ only), more than half of the neurons in each subset supplied the bladder (Dil+). An important factor to consider is that the second part of this study which examined histochemical changes in bladder NG neurons did not assess SP expression because that marker/channel was replaced with the red Dil tracer. Therefore, the percentages of bladder neurons in both the P2X3+ and IB4+ subsets after $\mathrm{SCl}$ are likely an overestimation. As a result, it would be possible that some of the neurons in those percentages would contain SP+ and SPneurons, which if included, could lower the overall numeric values. 
Plasticity related changes in bladder vagal afferents falls in line with evidence from the spinal system after SCl. Spinal sensory neurons innervating the bladder exhibit both morphological and physiological changes after SCl (Kruse et al., 1995, Yoshimura and de Groat, 1997). Given the important transduction role of P2X3 receptors in spinal bladder afferents (Cockayne et al., 2000) and the fact that many vagal neurons traced from the bladder expressed P2X3 suggests that the vagus nerve may participate in the sensory portion of micturition function. Our collective recent data indicating extensive vagal afferent innervation of mammalian urinary bladder (Herrity et al., 2014) and spinal cord injury-induced changes in a transduction channel like P2X3, may have important clinical applications. Such changes could contribute to whatever plasticity underlying reports of altered sensations stemming from the below level viscera, such as sensations of bladder filling or fullness in clinically complete $\mathrm{SCl}$ patients above T10 (Wyndaele, 1991, Komisaruk et al., 1997, Ersoz and Akyuz, 2004).

Even though IB4 binding decreased in a subset of NG neurons following injury, there are still spared IB4+ neurons that have the potential to sprout (Li and Zhou, 2001) although this phenomenon has yet to be demonstrated in vagal afferent neurons. A large proportion of the IB4 neurons were traced from the bladder which is complementary to an earlier study showing that IB4 binds different types of visceral afferents in the NG (Zhong et al., 2008). Although we did not trace from the distal urethra, a large proportion of spinal neurons which innervate this region of the lower urinary tract include IB4+ afferents (Yoshimura 
et al., 2003). Sprouting at the lumbosacral cord after transection injury from these afferents altered voiding efficiency, likely by contributing to detrusor-sphincter dyssynergia (Zinck and Downie, 2008). These studies in the somatosensory system are referenced in order to point out that while we identified many IB4+ bladder NG neurons that may have the chemical capability to sprout for example, the functionality of such a subset with these attributes has not been analyzed. 


\section{Table 4}

$\underline{\text { Immunohistochemical Reagents }}$

\begin{tabular}{|lllll|}
\hline Target & $\begin{array}{l}\text { Primary } \\
\text { Detection }\end{array}$ & $\begin{array}{l}\text { Dilution/Vendorl } \\
\text { Catalog \# }\end{array}$ & $\begin{array}{l}\text { Secondary } \\
\text { Detection }\end{array}$ & $\begin{array}{l}\text { Dilution/Vendorl } \\
\text { Catalog \# }\end{array}$ \\
\hline P2X3 & $\begin{array}{l}\text { GP-anti } \\
\text { P2X3 }\end{array}$ & $\begin{array}{l}\text { 1:1000/Neuromics/ } \\
\text { GP10108 }\end{array}$ & $\begin{array}{l}\text { Dky anti- } \\
\text { GP- } \\
\text { AF®488 }\end{array}$ & $\begin{array}{l}1: 100 / J a c k s o n / ~ \\
706-545-148\end{array}$ \\
\end{tabular}

AF, Alexa Fluor; Dky, Donkey; GP, Guinea Pig; HRP, Horseradish peroxidase; MS, Mouse; RBT, rabbit; SA, Strepavidin; SP, Substance P; TSA, Tyramide Signal Amplification 

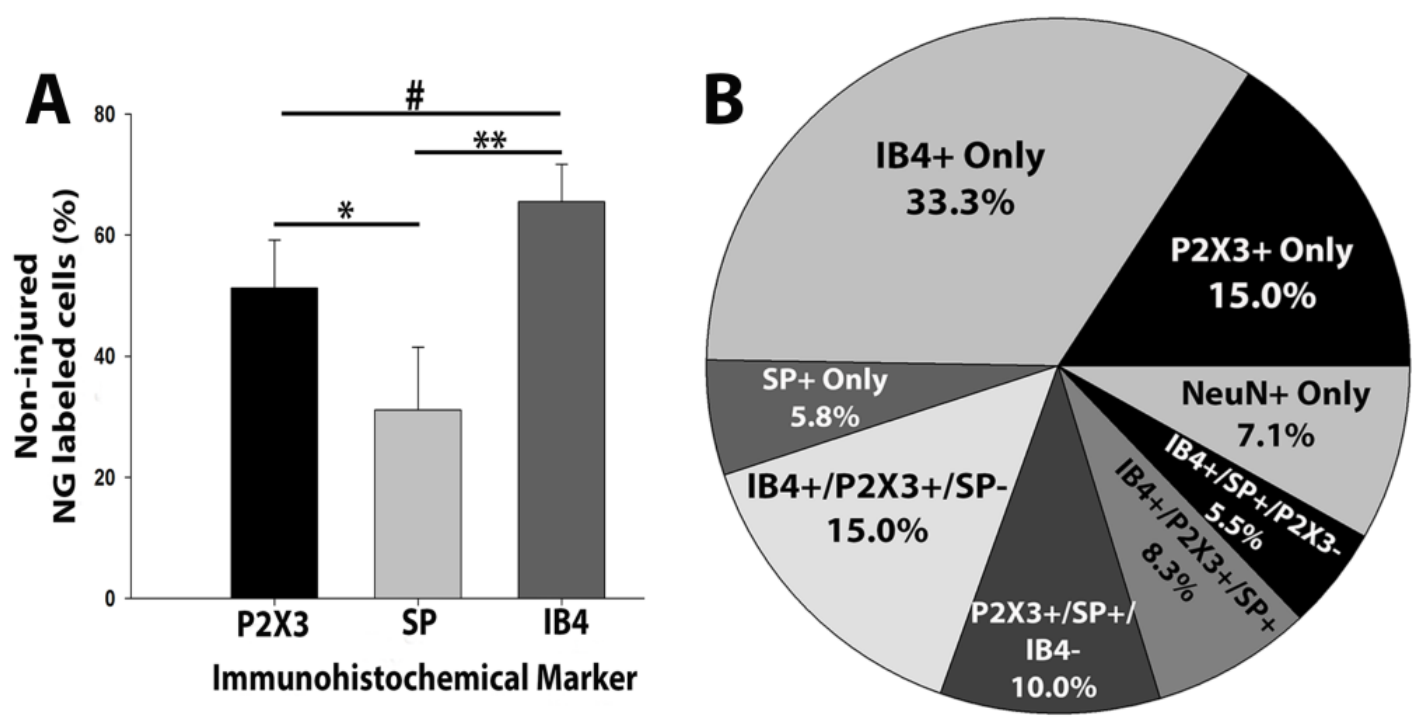

Figure 10 Immunohistochemical representation of P2X3, IB4 and SP in NG neurons

A. Following staining of the immunohistochemical markers P2X3, SP and IB4, the bar graph demonstrates that all three were well represented in the NG, with the majority being IB4+ (IB4 vs P2X3, \#p<.05; IB4 vs SP, ${ }^{\star \star} p<.001 ; \mathrm{P} 2 X 3$ vs SP, $\left.{ }^{*} \mathrm{p}<.01\right)$. B. A pie graph depicting all possible combinations of the molecular targets in the NG. Note that neurons that were IB4+ only were the most prevalent and most NG neurons were labeled with at least one of the three cellular targets examined. (One-way ANOVA with Tukey post hoc t-tests, $n=3,2$ ganglia, values are expressed as mean \pm S.D.) 


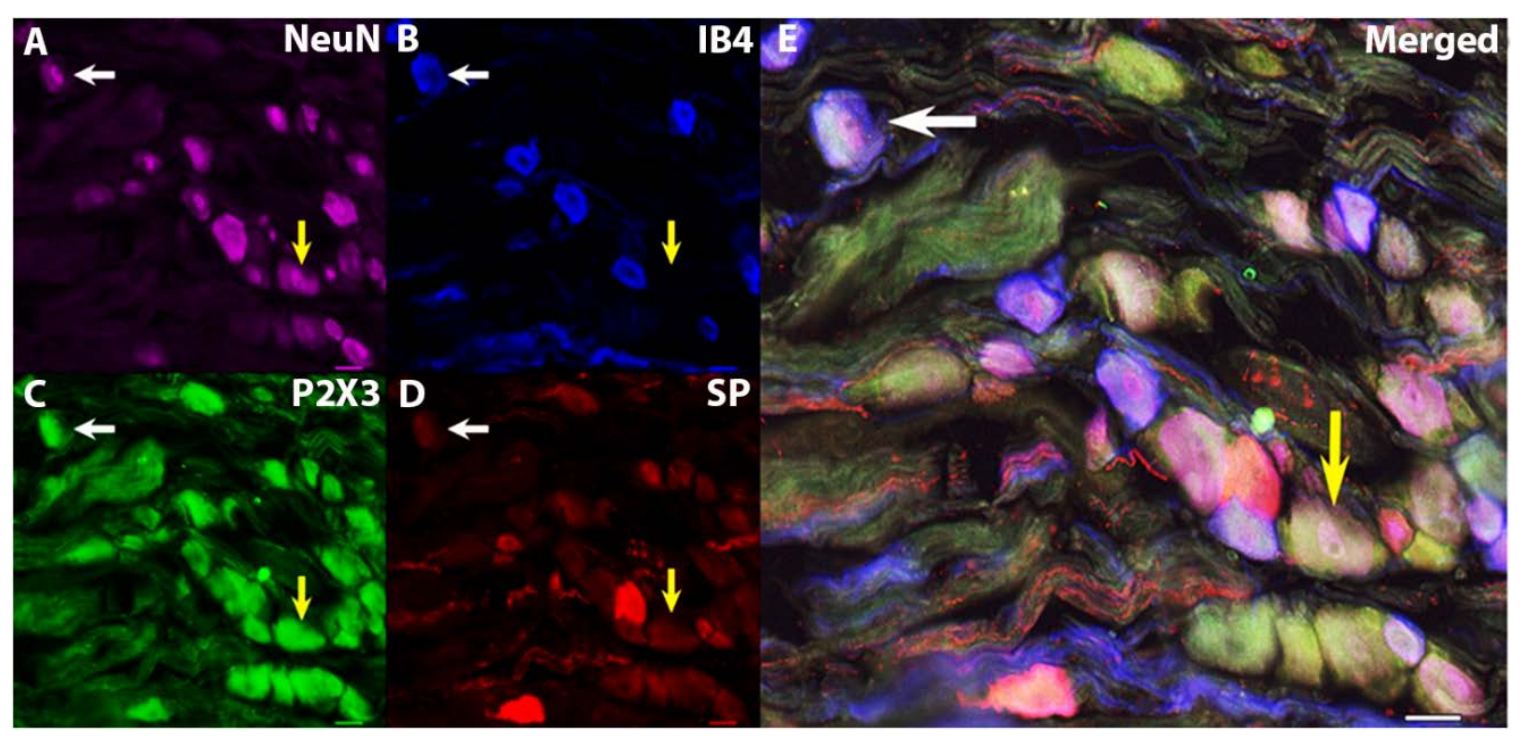

Figure 11 Quadruple immunohistochemical staining in the NG A confocal image displays the typical staining within the NG. NeuN was used to label all neurons. Different histochemical combinations include neurons that were IB4+, P2X3+, but SP- (white arrows) and neurons that were IB4-, P2X3+ and SP(yellow arrows). Scale bar indicates $25 \mu \mathrm{m}$. 


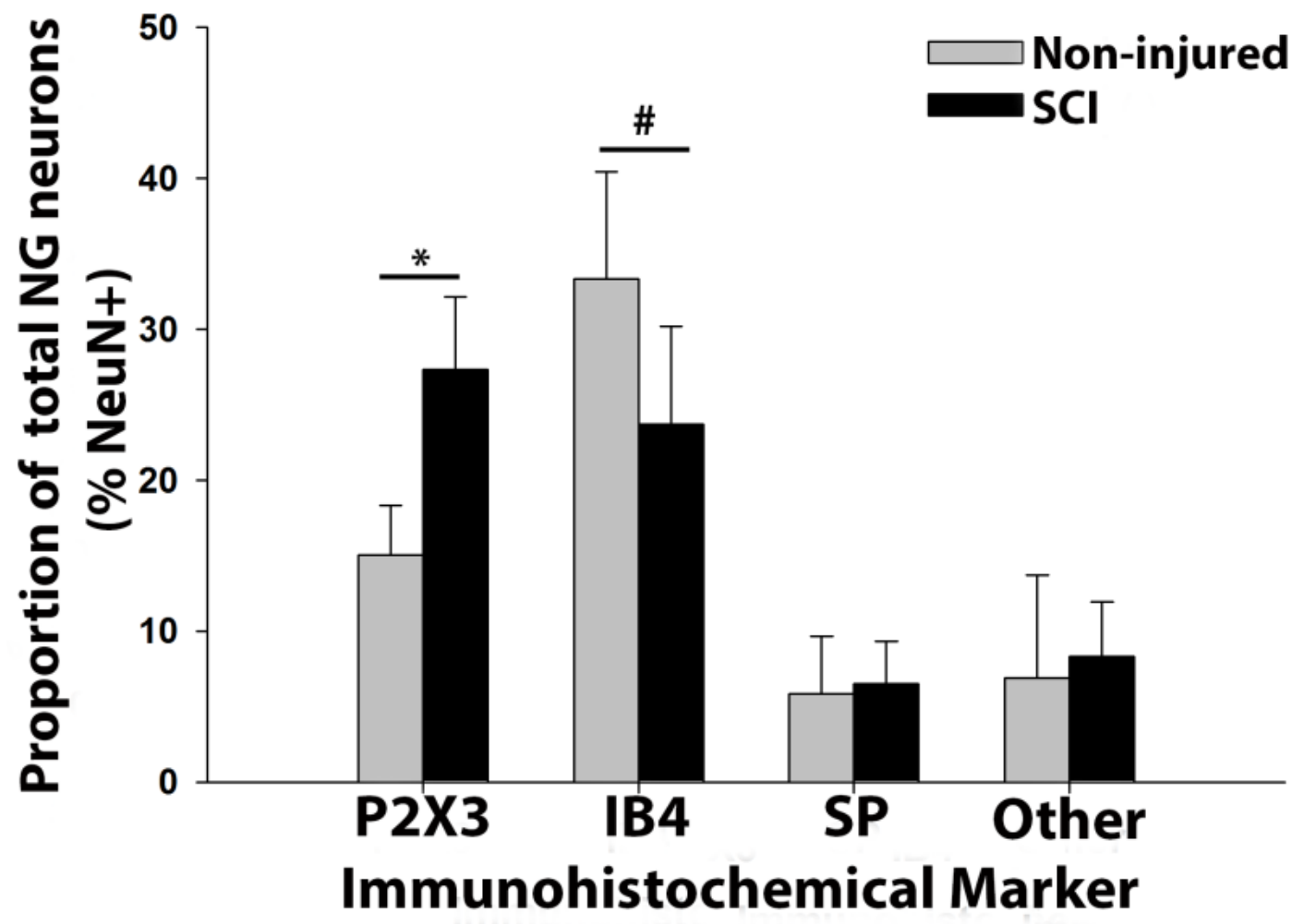

Figure 12 The effect of $\mathrm{SCl}$ on number of NG neurons expressing the individual markers

Following $\mathrm{SCl}$, there was an increase in $\mathrm{P} 2 \mathrm{X} 3$-ir in the transected group relative to intact/normal ( $\mathrm{SCl}, 27.3 \pm 4.8 \%$ versus non-injured, $\left.15.0 \pm 3.3 \%,{ }^{*} \mathrm{p}<.001\right)$ and a decrease in IB4 binding in the transected group relative to intact/normal $(\mathrm{SCl}$, $23.7 \pm 6.5 \%$ versus non-injured, $33.3 \pm 7.1 \%, \# p<.05)$. No changes were apparent for SP. The "Other" category represents neurons that were $\mathrm{NeuN+}$, but did not express or bind any of the markers. ( $N=6,12$ ganglia) 


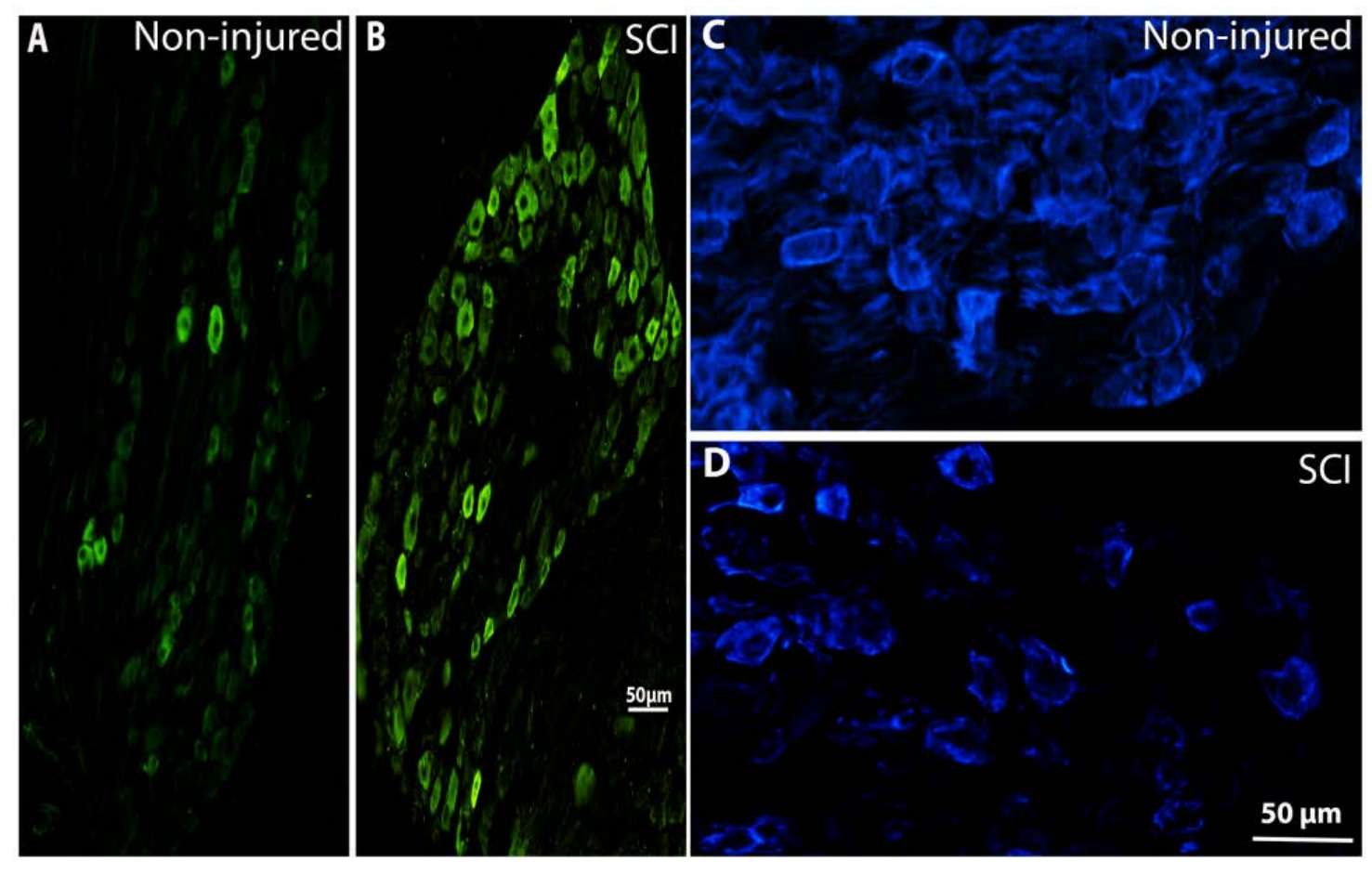

Figure 13 The effect of SCl on P2X3 and IB4 in the NG

An example displaying P2X3-ir (A) and IB4 binding (C) in the NG and following chronic spinal cord transection injury at T8 (B and D, respectively). Note the presence of increased P2X3-ir and decreased IB4 binding post-SCI. Images of sections from both $\mathrm{SCl}$ and non-injured animals were stained and captured with the same protocols and at the same time. 


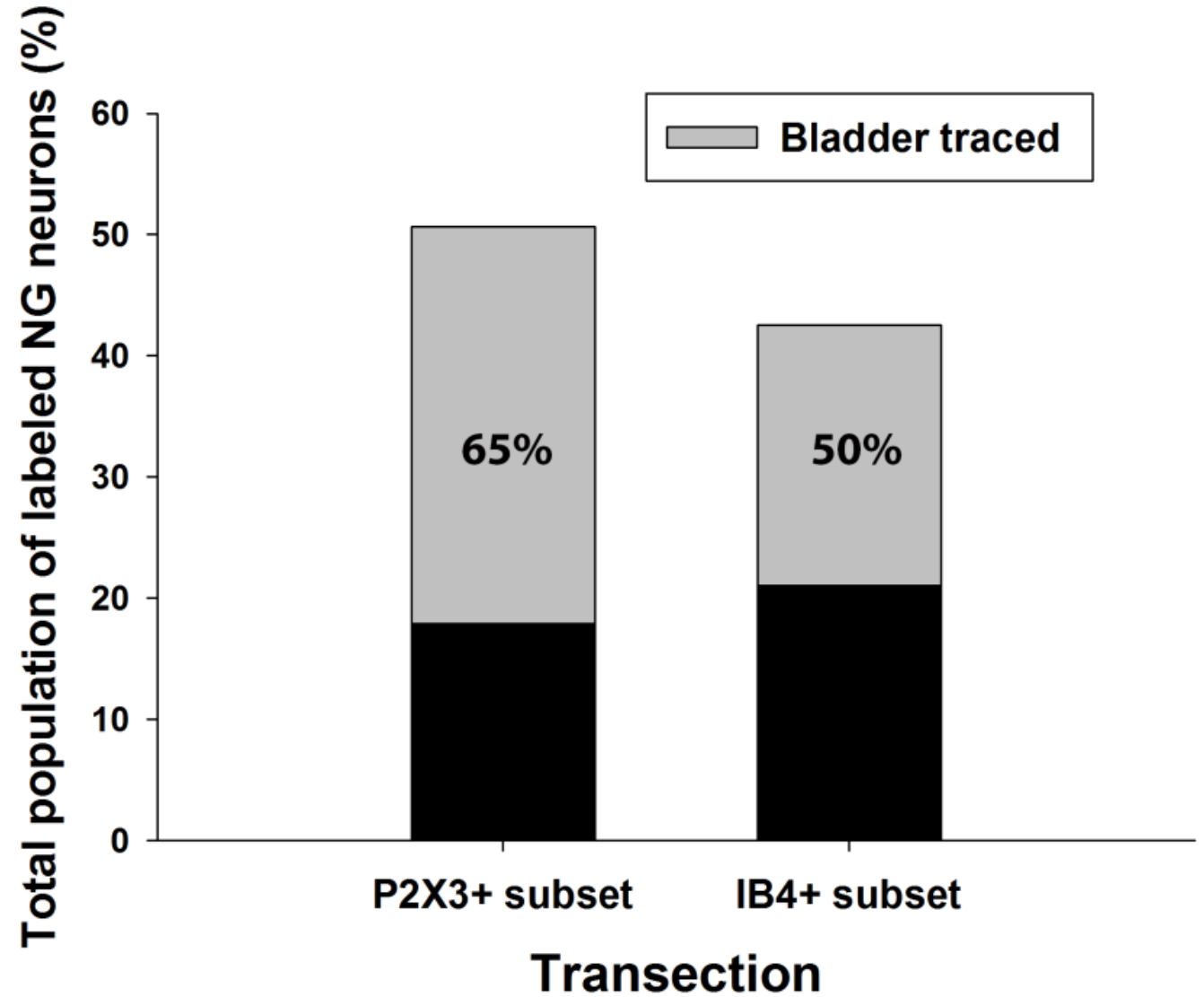

Figure 14 Effect of $\mathrm{SCl}$ on bladder-traced NG neurons Demonstrating that out of the total percentage of either P2X3 or IB4 subsets after injury, more than half of the neurons were traced from bladder. Bladder innervating neurons in the $\mathrm{P} 2 \mathrm{X} 3+$ subset represent $(32.8 \pm 1.1 \%)$ while in the IB4+ subset, they represent $(42.6 \pm 5.1 \%) .(\mathrm{N}=3,6$ ganglia $)$ 


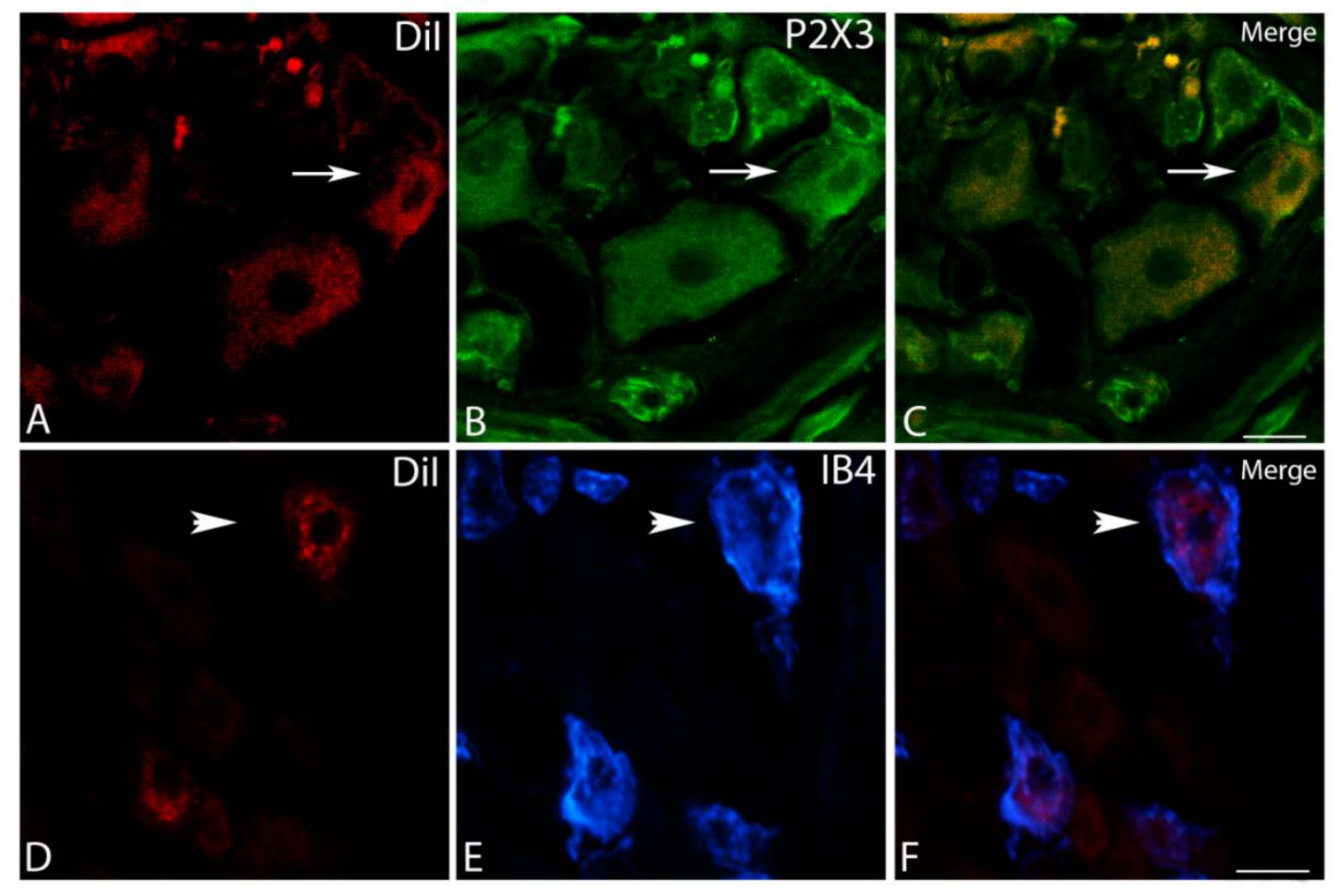

Figure 15 P2X3-ir and IB4 binding in bladder-traced NG neurons after transection A confocal image illustrating a Dil+ neuron in panel A that is also IR for P2X3 in panel B (white arrows). Panel C demonstrates the overlay. An image from the inverted Nikon microscope illustrating a Dil+ neuron in panel $D$ that also binds IB4 in panel $\mathrm{E}$ (white arrowhead). Panel $\mathrm{F}$ demonstrates the overlay. One example of each is displayed. In both images, the scale bar indicates $25 \mu \mathrm{m}$. 


\section{CHAPTER IV}

THE EFFECT OF SPINAL CORD INJURY ON THE ELECTROPHYSIOLOGICAL PROPERTIES OF NODOSE GANGLION NEURONS INNERVATING THE URINARY BLADDER 


\section{$\underline{\text { Introduction }}$}

Vagal afferent neurons of the nodose ganglia (NG) have receptors on their peripheral terminals that play an important role in responding to a wide variety of stimuli from most of the body's internal organs. Sensory information is transduced into neural activity and is transmitted to the central nervous system where it is integrated within the brainstem and processed in multiple regions. The central circuitries mediating visceral organ function include an efferent limb from the dorsal motor nucleus of the vagus that exits the brainstem back to the target organs. A signaling reflex that includes input to the NTS and output from the dorsal motor nucleus of the vagus is necessary for maintaining physiological homeostasis at different levels of gastrointestinal (Browning and Mendelowitz, 2003, Travagli et al., 2006, Holmes, 2012), cardiovascular (Aviado and Guevara Aviado, 2001) and respiratory (Jordan, 2001) control. Aside from receiving afferent innervation from the vagus nerve, most of the viscera also are dually supplied from spinal afferents (Burden et al., 1983, Gattone et al., 1986, Jancso and Maggi, 1987, Neuhuber, 1987, Springall et al., 1987, Altschuler et al., 1989, Neuhuber, 1989, Ortega-Villalobos et al., 1990, Berthoud and Powley, 1992, Kummer et al., 1992, Altschuler et al., 1993, Cheng et al., 1997, Komisaruk et al., 1997, Collins et al., 1999, Vizzard et al., 2000, Hubscher et al., 2004, Komisaruk et al., 2004, Herrity et al., 2014). A dual sensory supply may serve as a biologically important mechanism for relaying different types of visceral 
sensations. Information that is conveyed centrally by the vagus typically mediates functions that are below the level of consciousness, although intense stimuli after injury may be perceived as discomfort and/or pain (Janig, 1996). In fact, with many terminal endings in close contact to the mucosal lining of the viscera, vagal afferents appear to be conveniently located to detect noxious chemical information (Berthoud and Neuhuber, 2000).Thus, besides functioning as physiological sensors and serving an interoceptive role, vagal afferents may also play a role in noxious visceral signaling.

Although it is commonly thought that vagal afferents are not involved in the sensation of pain (Cervero, 1994, Ozaki et al., 1999), there is accumulating evidence that they partake in this process. For instance, gastric distention in the noxious range has been shown to result in a greater level of c-fos immunoreactivity (a marker of central neuronal activity following a peripheral stimulus) in the solitary nucleus compared to regions in the thoracic spinal cord that receive gastric input (Traub et al., 1996). It also has been proposed that activation of vagal afferents, whether through electrical or chemical means could contribute to changes in nociception through either facilitation or inhibitory modulation (Randich and Gebhart, 1992). Electrical stimulation of vagal afferent fibers has been shown to excite spinothalamic tract neurons in the cervical spine and was proposed to be a potential mechanism for referred pain that accompanies myocardial ischemia (Fu et al., 1992, Chandler et al., 1996). Furthermore, previous work from our lab demonstrated increased 
responsiveness in the medullary reticular formation to vagal nerve stimulation with chemical irritation of the bladder compared to mechanical distention of the bladder in the context of a complete thoracic spinal transection (Kaddumi and Hubscher, 2007).

Alterations to internal organs from injury, inflammation or even denervation secondary to trauma or disease states impacts the sensory neurons that innervate those tissues and can affect how information they convey is interpreted centrally in the nervous system. Subsequent changes to the microenvironment of the viscera have the potential to influence soma phenotype of sensory neurons and can lead to changes in afferent input and even contribute to the development of visceral hypersensitivity (Murphy et al., 1995, Neumann et al., 1996, Li et al., 1999, Michael and Priestley, 1999, Zhou et al., 1999, Banerjee et al., 2007, Banerjee et al., 2009a, Banerjee et al., 2009b, Hill et al., 2010, Wang et al., 2011). For instance, spinal cord injury (SCI) above the lumbosacral region results in significant impairments to both the neurological and histological integrity of visceral organs, such as the bladder, ultimately leading to global deficits in function. SCI disrupts voluntary and supraspinal control of micturition, initially triggering an a-reflexive bladder, followed by increased external urethral sphincter tone, urinary retention and the development of an automatic spinal voiding reflex (de Groat, 1995, de Groat and Yoshimura, 2006). Altered micturition results from the bladder and external urethral simultaneously contracting, referred to as detrusor-sphincter dyssynergia (de Groat and 
Yoshimura, 2010). Over time, post-SCI changes to the lower urinary tract create a system of high intravesical pressure, detrusor muscle overactivity as well as a disruption of the bladder urothelial lining (de Groat et al., 1990, Apodaca et al., 2003).

It also has been suggested that $\mathrm{SCl}$ triggers phenotypic changes in the bladder afferent pathways to the spinal cord, including soma hypertrophy (Kruse et al., 1995, Yoshimura and de Groat, 1997, Yoshimura et al., 1998), neurochemical alterations (Vizzard, 2000b, Zvarova et al., 2005, Vizzard, 2006, Zinck et al., 2007, Zinck and Downie, 2008) and electrophysiological changes (Yoshimura et al., 1996, Yoshimura and de Groat, 1997). These SCl-induced phenomena may explain the bladder overactivity or hyperreflexia that appears post-injury (Yoshimura, 1999). Additional target-neural interactions, mediated by neurotrophic factor release from the urothelial lining in response to a hypertrophied detrusor muscle or release from the spinal cord, also can influence innervating neurons (Seki et al., 2002, Seki et al., 2004, Vizzard, 2006).

The results from Chapter III of this dissertation indicate that $\mathrm{SCI}$ triggers a phenotypic switch in NG neurons as well as in NG neurons innervating the bladder. In both populations, there are significant increases in the number of neurons expressing P2X3 and decreases in the number of neurons binding IB4. More than half of the bladder-innervating NG neurons displayed these molecular changes. To further understand the functional capability of bladder-vagal afferents, electrophysiological properties were examined using whole cell patch- 
clamp methods. It is hypothesized that $\mathrm{SCl}$ results in increased excitability in NG neurons innervating the bladder, as measured by in vitro cellular recordings.

Changes in visceral sensitivity (i.e. hypersensitivity) can contribute to disorders in vagal afferent processing and affect the overall homeostatic state of the viscera. 


\section{Methods}

Animals

All experimental procedures were carried out according to NIH guidelines and protocols reviewed and approved by the Institutional Animal Care and Use Committee at the University Of Louisville School Of Medicine. This study used 612 week old male Wistar rats ( $\mathrm{n}=16$, Harlan Sprague Dawley, Inc, Indianapolis, IN) which were approximately 175-250 grams in weight at the time of tracer injection and SCl and 350-375 grams in weight when euthanized for in vitro NG electrophysiology recordings. All animals were individually housed in an animal room with a 12-hour light and dark cycle. They had ad libitum access to water and food (Laboratory Rodent Diet). Groups were either naïve $(n=8)$ or spinal cord injured $(n=8)$. Each group received retrograde neural tracer injected into the bladder to enable identification of single NG neurons which innervated the bladder.

\section{Spinal cord injury}

At six weeks of age, a subset of animals $(n=8)$ for spinal transection were anesthetized with a mixture of ketamine $(80 \mathrm{mg} / \mathrm{kg})$ and xylazine $(10 \mathrm{mg} / \mathrm{kg})$, injected intraperitoneally. All surgeries were performed under aseptic conditions and the body temperature was maintained within the range of $36-37^{\circ} \mathrm{C}$ via a warm water recirculator (Gaymar T/Pump, Gaymar Industries Inc, Orchard Park, NY) throughout the surgery and recovery period. Following our previously 
published protocol (Kaddumi and Hubscher, 2007), a dorsal longitudinal incision was made to expose the T7 vertebra and a laminectomy was performed in order to expose the underlying T8 spinal cord. The overlying dura was reflected laterally and the spinal cord cut using a pair of surgical microdissecting scissors. Gentle suction with an air vacuum was used to carefully elevate the cut stump in order to verify the completion of the lesion. Gelfoam (Pharmacia \& Upjohn Company, Kalamazoo, MI) soaked in topical hemostat solution (Henry Schein Inc., Melville, NY) was placed in the lesion cavity. The incision was closed using 4-0 nylon suture for the muscle layers and fascia and surgical clips for the skin. Animals were given subcutaneous injections of ketoprofen (Ketofen, $2.5 \mathrm{mg} / \mathrm{kg}$, Fort Dodge Animal Health, Fort Dodge, IA) for analgesia twice a day for 2 days, $0.5 \mathrm{ml}$ of dual penicillin (Penicillin G coupled with Procaine, PenJect ${ }^{\circledR}$, The Butler Company, Columbus, $\mathrm{OH}$ ) in a single dose peri-operatively as a general prophylactic and $5 \mathrm{mg} / \mathrm{kg}$ gentamicin (GentaFuse ${ }^{\circledR}$, Butler Schein, Dublin, $\mathrm{OH}$ ) once per day for 5 days to prevent bladder infections. After surgery each animal was housed individually. The urinary bladder was expressed every 8 hours until the micturition reflex occurred automatically, 6-12 days (Hubscher and Johnson, 2000). Animals survived for six weeks followed by euthanasia and NG tissue removal, which was immediately prepared for in vitro recordings. Note that the age-matched non-injured rats survived the same time frame as the $\mathrm{SCl}$ rats.

Retrograde tracer injection 
At 10-11 weeks of age, all animals were anesthetized with a mixture of ketamine $(80 \mathrm{mg} / \mathrm{kg})$ and xylazine $(10 \mathrm{mg} / \mathrm{kg})$. They received a ventral/caudal midline peritoneal incision to expose the urinary bladder which was subsequently manually voided by pressure. Using an established protocol (Rau et al., 2007, Herrity et al., 2014), the fluorescent tracer FAST Dil $^{\text {TM }}$ oil (1,1'-dilinoleyl-3,3,3',3',tetramethylindo-carbocyanine perchlorate, $5 \mathrm{mg}$ dye dissolved in $0.1 \mathrm{ml}$ methanol, Molecular Probes, Inc., Eugene, OR) was injected into the bladder wall with a dye-dedicated 33-gauge needle coupled to a Hamilton microsyringe (Fisher Scientific, Pittsburgh, PA). Note that the abbreviation Dil is used throughout the text/figures/legends to refer to FAST Dil ${ }^{\mathrm{TM}}$ oil. Dye injections were made to the circumference of the trigone, body and dome areas $(10 \mu$ l volume per animal divided into 10 injections of $1 \mu$ l each; (Herrity et al., 2014)). Animal body temperature was maintained within the range of $36-37^{\circ} \mathrm{C}$ during surgery via a warm water recirculator (Gaymar T/Pump, Gaymar Industries Inc, Orchard Park, NY). After each injection, the needle was removed slowly and any dye-leakage was removed by cotton-tipped applicators. After injections were completed, the exposed viscera were hydrated as necessary with $5 \%$ Dextrose Lactated Ringers, the abdominal musculature was sutured closed (Ethicon 4-0 nonabsorbable surgical suture), the skin closed with Michel clips (Fine Science Tools, Foster City, CA), and a topical antibiotic (Bacitracin, Actavis Mid Atlantic LLC, Lincolnton, NC) applied. Following surgery, animals were placed on a heating pad and core temperature monitored. Post-operative medication was 
provided as per spinal transection surgery. All animals were monitored daily to inspect the surgical incision and identify any changes in an animal's general condition.

Preparation of cells for patch-clamp electrophysiology

NG extraction methods (Herrity et al., 2014) and cellular dissociation procedures (Petruska et al., 2000c, Petruska et al., 2002) have been described previously. Briefly, animals were deeply anesthetized with a ketamine $(80 \mathrm{mg} / \mathrm{kg}$ body weight)/xylazine $(10 \mathrm{mg} / \mathrm{kg})$ mixture and transcardially exsanguinated with heparinized phosphate buffered saline. Each vagus nerve was identified adjacent to the carotid artery, gently separated from surrounding tissues, and traced rostrally to the NG, which was excised and desheathed. Bilateral ganglia were digested in a tube containing dispase II (neutral protease, $5 \mathrm{mg} / \mathrm{ml}$; Roche) and collagenase type $1(2 \mathrm{mg} / \mathrm{ml}$; Worthington). The tube was incubated in a circulating water bath $\left(37^{\circ} \mathrm{C}\right)$ for $90 \mathrm{~min}$. During this time frame, the cells were then gently triturated every 30 minutes to dissociate them. The cells were removed and then washed and plated on 8-10 poly-I-lysine (Sigma)-coated petri dishes. Dishes were kept in an aerated holding bath for at least $2 \mathrm{~h}$ before use. The holding bath was removed from ambient light to prevent any potential photobleaching of dye-containing cells. All recordings were made at room temperature $\left(22-23^{\circ} \mathrm{C}\right)$ and were completed within $10 \mathrm{~h}$ of $\mathrm{NG}$ retrieval from an animal. 


\section{Solutions}

Plated cells were placed in Tyrode's solution containing $140 \mathrm{mM} \mathrm{NaCl}, 4$ $\mathrm{mM} \mathrm{KCl}, 2 \mathrm{mM} \mathrm{MgCl} 2,2 \mathrm{mM} \mathrm{CaCl}_{2}, 10 \mathrm{mM}$ glucose, and $10 \mathrm{mM}$ HEPES, adjusted to $\mathrm{pH} 7.4$ with $\mathrm{NaOH}$. The recording electrodes were filled with $130 \mathrm{mM}$ K-gluconate, 2 mM Mg-ATP, $0.3 \mathrm{mM}$ Tris-GTP, $11 \mathrm{mM}$ EGTA, 1 mM CaCl , 1 $\mathrm{mM} \mathrm{MgCl} 2$, and $10 \mathrm{mM} \mathrm{HEPES}, 7 \mathrm{mM} \mathrm{KCL}, 2 \mathrm{mM} \mathrm{NaCl}$, adjusted to $\mathrm{pH} 7.4$ with $\mathrm{KOH}$; Osmolarity was between 315-325 mOsm.

\section{Whole cell patch recording}

The electrophysiological recording techniques used in this study have been previously described in detail (Petruska et al., 2000c, Petruska et al., 2002). Electrodes were prepared (1.8-4.2 M $\Omega$ ) from glass pipettes using a Brown and Flaming-type horizontal puller (Sutter model P87). Each petri dish was briefly illuminated using epifluorescence microscopy to reveal Dil-labeled cells (total exposure of field $<1 \mathrm{~min})$. Only intensely fluorescent cells were considered positive and only one cell was recorded per dish. Whole cell recordings were made with an Axopatch 200B (Molecular Devices). Stimuli were controlled and digital records captured with pClamp8.1 software and a Digipack 1322A converter (Molecular Devices). Series resistance $\left(R_{\mathrm{S}}\right)$ was compensated 30$60 \%$. Whole cell resistance was assessed by pClamp software from voltage transients associated with small step commands (10 mV). After a recording was 
completed, digital images of the brightfield and fluorescent fields of view (545nm) were captured using a Dage MTI RC300 camera coupled to a PC running Scion Image 4.0.2. All experiments were conducted at room temperature. Only cells having a resting membrane potential (RMP) of -40 to $-70 \mathrm{mV}$ were included in this study.

AP generation and measurements

Action potentials were evoked at threshold through a 1-ms, 1,500- to 5,000-pA current step. The average of three action potentials was used to determine afterhyperpolarization (AHP) and action potential duration at the base (APDb). To quantify AHP, a criterion of $80 \%$ recovery to baseline was used (AHP80) (Djouhri et al., 1998)). All electrophysiological measurements were performed using Clampfit analysis software. APDb was measured as the time from the first upward deflection of the AP waveform to its return to baseline ($60 \mathrm{mV})$. Currents were normalized by dividing by whole cell capacitance, an indicator of cell size $(p F)$. Rates of decay $(\tau)$ were determined for peak currents using the equation $f(t)=A i \exp (-e-t / r i) \ldots+C$, where $A$ is amplitude and $C$ is capacitance. Single and multiple exponential fits were examined.

\section{Statistics}

Due to extreme ranges of values in the data, analyzing the total set as a group results in high variability. As a result, any comparisons with that data are 
not significant. Graphing the data revealed a significant bi-modal distribution. Therefore, we used the median of the entire data set to establish high and low responders, since it was not appropriate to analyze the data with parametric statistics of means and standard deviations (Hays, 1981). Briefly, the grand median was acquired for the entire AP frequency-current data set. Based on the grand median, data were categorized into high responders ( $\geq 6 \mathrm{APs} /$ current step) and into low responders (<6 APs/current step). An independent-sample median test was applied for a comparison of high and low responders as a whole. A repeated measure mixed model analysis of variance (ANOVA) with Tukey's post hoc $\mathrm{t}$-tests was performed for evaluating group differences (high and low responders in both the non-injured and $\mathrm{SCl}$ groups) for current-frequency curves. Binomial proportions and Student's t-tests were used to examine the possibility of significant differences between the electrophysiological characteristics of selected groups. Pearson correlations were performed for evaluating degrees of linear dependence between electrophysiological variables of selected groups. For all tests, a difference was considered significant if $p \leq$ to 0.05 . Results are reported as means $\pm S D$. 
Results

Identification of bladder neurons in the NG

The retrograde tracer Dil was injected into lining of the bladder (FIG 16A) in order to identify bladder-innervating neurons in the NG. Dil+ neurons were readily detected and demonstrated a punctate cytoplasmic pattern of staining (FIG 16B) evident through epifluorescence illumination.

Electrophysiological characteristics of NG neurons innervating the bladder

Whole cell patch-clamp recordings of 33 intensely labeled Dil+ NG neurons (FIG 17) were subsequently made from 16 animals (32 extracted NGs/bilateral ganglia used per experiment). In order to examine the features of individual APs, brief (1-ms) depolarizing current steps were used to evoke single APs (Figure 18). The AP peak (from RMP -mV), overshoot (above $0 \mathrm{mV}$ ), AHP and AHP 80\% recovery, as well as APDb from all bladder-innervating NG cells were quantified, and the values are displayed in Table 5. Additional anatomical and electrophysiological characteristics, including, cell diameter $(\mu \mathrm{m})$, cell capacitance $(p F)$, tau $(\mu s)$, membrane resistance $(M \Omega)$, rheobase $(p A)$ and peak instantaneous frequency (IF) (Hz) also are provided.

Among the total number of non-injured bladder-innervating NG cells, AP frequency patterns revealed two significant subsets. Cells could be identified as either high responders (displaying $\geq 6$ action potentials (APs) evoked throughout 
the duration of a 1-ms current step) or low responders (displaying $<6$ action potentials evoked throughout the duration of the 1-ms current step). There was a significant difference in the distributions of individual high and low responders in the non-injured group across 300pA-1000pA steps of current injection (Figure 19). Neurons from the non-injured high responder (NHR) group displayed significantly greater AP firing frequency at consecutive points of current injection compared to neurons from the non-injured low responder (NLR) group (Figure $20, p<.05)$. Note that the average total number of APs fired across the range of current steps $(0-1000 \mathrm{pA})$ for the NHR $(15.78 \pm 10.8)$ was significantly greater than the average number of APs fired for the NLR $(1.30 \pm 0.6, p<.01)$. Average rheobase, or the minimum amount of current required to elicit an AP between the two non-injured subsets also was significant (NHR, $85.00 \pm 41.2$ pA versus NLR, $175.0 \pm 88.0 \mathrm{pA}, \mathrm{p}<.05$ ). However, peak instantaneous frequency (capacity of the cell to fire sequential APs) was not significantly different between the two subsets (NHR, $42.04 \pm 17.0 \mathrm{~Hz}$ versus NLR, $25.43 \pm 17.9 \mathrm{~Hz}$ ).

\section{Effects of SCl on NG neurons innervating the bladder}

In relation to the non-injured group, spinal transection injury did not cause a significant difference in the average number of bladder-labeled cellular recordings per experiment (Non-injured, $5.25 \pm 2.9$ versus SCI, $5.75 \pm 2.1$ ). SCl also did not result in a significant difference in resting membrane potential of bladder afferent NG neurons compared to the spinally intact group. There were 
no significant differences in AHP trough, AHP80 (80\% recovery to RMP), AP duration (width) or rheobase (with respect to the 2 groups overall, Non-injured vs $\mathrm{SCl})$ due to $\mathrm{SCl}$ (Table 5). However, the membrane resistance (Rm) was significantly greater and the mean depolarizing threshold for eliciting an AP was significantly greater in the transected group as a whole (Table 5).

Following recordings of Dil+ NG neurons from animals receiving chronic spinal transection, two distinct populations of high and low responders, (SHR and SLR, respectively), also were evident, as significant differences in their distribution plots across all current steps occurred (from 100pA-1000pA, Figure 19). Interestingly, the SHR group and NHR both displayed similar AP frequencycurrent curves, but at the midway point of the current step series, the SHR group exhibits declining mean AP frequency, whereas the NHR group AP frequency plateaus (Figure 20). A comparison of the AP frequency-current curves between the two high responder groups (NHR vs SHR) was not statistically significant.

The SHR group demonstrated significantly greater than average overall AP frequency compared to the SLR group (SHR, $14.22 \pm 8.2$ versus SLR, $0.80 \pm$ $0.2, p<.05)$. The SHR group also displayed a significantly lower than average rheobase compared to the SLR subset (SHR, $82.14 \pm 66.8$ versus SLR, $216.67 \pm$ 175.6 Hz, p<.05). Peak instantaneous frequency for the SLR group was nonapplicable since AP firing was never greater than one and thus comparisons for this criterion between the subsets could not be made. A representative example of an $\mathrm{SCl}$ low responder pattern compared to a high responder pattern is 
provided in Figure 21. A comparison of mean AP threshold revealed significant differences not only between non-injured and SCI groups as whole (Table 5), but even with respect to the high and low responder subsets, significant differences were apparent. Regardless of AP frequency, $\mathrm{SCl}$ resulted in a lower than average mean threshold for firing an AP (Figure 22). Overall, the proportion of high to low responders within the $\mathrm{SCl}$ group was significantly greater compared to the proportion in the non-injured group (Figure 8, $p<.001$ ). 


\section{Discussion}

This study utilized in vitro electrophysiology to examine passive and active cell membrane properties of bladder-innervating NG neurons and assess how those electrical properties are affected by SCl. Initial data in non-injured bladderinnervating NG neurons revealed two distinct subsets that were roughly in equal proportions, high and low responders. These subsets also were apparent in the $\mathrm{SCl}$ group, and the proportion of high-to-low responders was significantly increased in the $\mathrm{SCl}$ group. Overall, $\mathrm{SCl}$ resulted in a greater membrane resistance and higher AP threshold compared to neurons from the spinally intact group.

\section{Retrograde tracer injections}

The use of Dil has shown to be an effective retrograde tracer of visceral afferents for in vitro procedures in both the NG and DRG (Dang et al., 2005, Rau et al., 2007, Dang et al., 2008, Grabauskas et al., 2010, Rau et al., 2014) and in a spinal transection model (Takahashi et al., 2013). We previously demonstrated that Dil injections into the lining of bladder results in a clearly labeled population of bladder-innervating NG neurons (Herrity et al., 2014). Despite the fact that the enzymatic process of dissociation likely results in a partial loss of neurons, in this study, Dil yielded a substantial population of intensely labeled cells to sample from. Importantly, Dil negative neurons were noted, indicating that the lipophilic 
tracer did not spread non-specifically. Prior controls for Dil from our lab (Herrity et al., 2014) and of others (Dang et al., 2008) suggest this tracer can be reliably used to label visceral afferents and remain stable when acutely dissociated for whole cell patch-clamp experiments. Finally, the number of Dil+ cells did not differ between the spinally intact and $\mathrm{SCl}$ groups, suggesting that there was not an overall loss of vagal neurons after transection injury. It should be noted, however, that since rats received multiple procedures ( $\mathrm{SCl}$ followed 5 weeks later by tracer injections), a conditioning or priming effect from the second manipulation could occur, thereby influencing our electrophysiological results. Injured sensory DRG neurons that have been repeatedly manipulated after CNS injury, developed an intrinsic growth capacity, which is sustained to enhance the regeneration of injured axons (Neumann et al., 2005)

\section{Electrophysiological properties of NG neurons}

Early electrophysiological recordings of nodose ganglion neurons in different species have previously documented three general populations based off action potential characteristics and conduction velocities in an effort to classify them as either A-type (myelinated) or C-type (unmyelinated) or more recently Ahtype (myelinated, fast CV) (Jaffe and Sampson, 1976, Gallego and Eyzaguirre, 1978, Stansfeld and Wallis, 1985, Puizillout and Gambarelli, 1989, Li and Schild, 2007). Although it is noted that these criteria do not provide a strict classification of neuronal type, they do demonstrate that the NG contains a heterogenous 
mixture of neurons (Schild et al., 1994). Along those lines, findings from the patch-clamp recordings in this study of bladder-innervating neurons also revealed distinct subsets based on AP frequency within both the intact and $\mathrm{SCl}$ populations. Although we did not classify A-, Ah- and C-type neurons, as this study did not maintain intact vagal afferent fibers from which conduction velocity and afferent fiber type can be determined, many C-type neurons were apparent. Most recorded neurons displayed characteristic electrical properties typical of this subtype as reported in the literature, such as a longer AP duration ( $>4 \mathrm{~ms})$, a longer AHP recovery (75-250ms) and reflecting the classical "hump" present on the repolarization phase (Ikeda et al., 1986, Schild et al., 1994). It is noted however, that a small proportion of these C-type neurons we observed could be of the myelinated Ah-type. They also display a delay on the repolarization phase, but maintain a higher conduction velocity than the C-type and therefore, have the potential to be miscategorized if classification is based on AP features ( $\mathrm{Li}$ and Schild, 2007, Lu et al., 2013). It has been suggested that the repolarization hump is primarily dependent on the degree of $\mathrm{Ca}^{2+}$ current, but also may be influenced by $\mathrm{Na}^{+}$ion currents of sufficient magnitude (Gallego and Eyzaguirre, 1978, Stansfeld and Wallis, 1985).

In agreement with reports in the literature, we found that vagal afferents with projections to the bladder, have little to no spontaneous or baseline activity (Gebhart et al., 2002, Browning and Mendelowitz, 2003). Vagal afferents in general also appear to display a varied or non-uniform level of passive and active 
membrane properties (Undem and Weinreich, 1993, Mendelowitz et al., 1995). In this study, the two distinct subsets in both groups (non-injured and SCI) were evident based on AP frequency over increasing increments of current injection. From the onset, the collected data set appeared to contain a great deal of variability (i.e. neurons exhibiting numerous APs and neurons exhibiting single APs). The ionic basis for increased AP frequency in the high responder subsets in this study is undetermined. However, repetitive firing properties have been previously reported in the literature of intact NG neurons (Stansfeld and Wallis, 1985) and it is thought that this characteristic is influenced by the transient outward current of voltage-gated $\mathrm{K}+$ channels. Alternatively, repetitive AP firing may be caused by levels of both intra- and extracellular $\mathrm{Ca}^{2+}$ concentration that may indirectly influence membrane potassium channels (Smith et al., 1983, Schild et al., 1994).

Another explanation for the increased AP frequency may be attributed to the adaptation response of vagal nerve endings to a stimulus (Jaffe and Sampson, 1976). For instance, mechanosensitive vagal afferents innervating the lungs have been shown to fall into either slowly adapting receptors (a burst of APs which are maintained as long as the stimulus is present) or rapidly adapting receptors (a brief burst of APs, after which, they rapidly adapt and may cease firing) (Knowlton and Larrabee, 1946, Widdicombe, 1954). Earlier research has shown that the adaptation response which underlies these two vagal populations in the lungs highly correlates with embryological origin (nodose versus jugular) 
(McAlexander et al., 1999). Afferent fibers from the placode-derived nodose ganglion, predominately rapidly adapt, whereas the majority of fibers that originate from the neural crest-derived jugular ganglion adapt slowly (McAlexander et al., 1999). It is noted that the adaptation response initiated at terminal endings does not always equate with the same electrophysiological response present at the soma (McAlexander et al., 1999). This feature may be particularly relevant when considering the use of pharmaceuticals as ionic channel blockers for studies/therapies. If embryological origin has such a strong influence on response profiles, it may predict how they respond to a treatment (i.e. vagal nerve stimulation).

Since subsets of neurons in both the non-injured and SCI populations exhibited increased AP frequency patterns, it is unclear whether or not these properties are attributed injury/stress (spinal transection, tracer injection or dissociation procedures) and/or if interaction with the bladder target tissue contributed. Assessment of the expression of activating transcription factor-3 (ATF-3), which is induced de novo in sensory and motor neurons following peripheral nerve injury and absent from intact neurons (Tsujino et al., 2000), may help determine if the experimental procedures incorporated in this study influenced the electrophysiological properties of the Dil+ NG neurons. Initially, all neurons sustain a peripheral as well as central injury from dissociation. However, all recordings are performed within 10 hours of NG extraction and therefore it is unlikely at this stage that these neurons would be undergoing ATF-3 induction, 
since its upregulation has been shown to occur around 14-24 hours after injury (axotomy, in DRG) (Averill et al., 2004, Rau et al., 2014). Since ATF-3 also is induced by cellular stress/damage, its expression in the NG SCI population may help provide an explanation for the observed decline of the SHR currentfrequency curve around 600pA (Hai et al., 1999, Allen-Jennings et al., 2001, Okamoto et al., 2001, Kawauchi et al., 2002, Hartman et al., 2004). It is possible however, that the SHR subgroup is displaying a rapidly adapting quality and that the observed decline in AP firing is reflective of a modified or fading signal rather than an absolute response to stimulus intensity.

Earlier intracellular recordings from an intact or in situ preparation of vagal ganglia fibers demonstrate similar membrane properties to patch-clamp recordings of dissociated neurons, suggesting that the procedure of dissociation does not induce excitability, but rather, the electrical properties seen reflect their physiology (Gallego and Eyzaguirre, 1978, Stansfeld and Wallis, 1985). It is likely that fluorescent exposure did not alter membrane parameters (Christian et al., 1993, Yoshimura et al., 1994), as control recordings from non-injured Dil negative neurons maintained similar passive and active membrane properties compared to non-injured Dil+ neurons (data not shown here).

No significant differences were found for cell size or capacitance indicating that $\mathrm{SCl}$ did not induce soma hypertrophy or an increase in membrane surface area. These passive membrane properties have the potential to influence active properties such as AP duration and threshold for AP generation (Titmus and 
Faber, 1990). We did find however, that SCI required a significantly higher depolarizing threshold in both the high and low responder subsets to elicit an AP. This higher AP threshold indicates that these bladder-innervating vagal neurons are less excitable after SCl. Evidence of decreased vagal afferent activity has come from the work of Homes et al., in which they show that following a high thoracic SCI (T3), NTS neurons demonstrate diminished vagal afferent sensitivity to gastrointestinal neuropeptides (Tong et al., 2011). It has been suggested that even though the vagal neurocircuitry remains intact after $\mathrm{SCl}$, there appears to be an overall hyposensitivity of vagal afferent neurotransmission to the brainstem (Holmes, 2012).

In this study, $\mathrm{SCl}$ also resulted in an increase in membrane resistance $(\mathrm{Rm})$, suggesting fewer reduced leak currents. A combination of increased Rm as well as a low rheobase (apparent for both the NHR and SHR subsets) suggests that less current is required to excite the bladder-innervating NG neurons after $\mathrm{SCl}$. Alternatively, neurons in the $\mathrm{SCl}$ group required a greater depolarization threshold to generate an AP, thereby exhibiting less excitability. Overall, the AP firing patterns present here may be regulated by the proportion of ion channels. The various types of ion channels and their kinetics differentially contribute to current flow across the membrane and can affect overall excitability. In order to clarify the mechanism underlying the changes seen after $\mathrm{SCl}$, future studies with a focus on voltage-gated $\mathrm{Na}+$ and $\mathrm{K}+$ channels should be examined. 


\section{Table 5}

Electrophysiological properties of NG bladder neurons

\begin{tabular}{|c|c|c|}
\hline & Non-Injured & $\mathrm{SCl}$ \\
\hline No. cells/rats & $16 / 8$ & $17 / 8$ \\
\hline Cell Diameter $(\mu \mathrm{m})$ & $31.87 \pm 4.89$ & $32.36 \pm 1.16$ \\
\hline Input Capacitance (pF) & $50.15 \pm 14.88$ & $49.40 \pm 5.09$ \\
\hline Membrane Resistance (M $\Omega$ ) & $379.27 \pm 245.85$ & $785.99 \pm 491.30 *$ \\
\hline Tau ( $\mu s)$ & $124.92 \pm 57.71$ & $112.89 \pm 19.00$ \\
\hline RMP (mV) & $-52.68 \pm 5.62$ & $-51.52 \pm 1.42$ \\
\hline \multicolumn{3}{|l|}{ Action Potential: } \\
\hline Peak (mV) & $107.36 \pm 13.78$ & $100.65 \pm 2.80$ \\
\hline Overshoot (mV) & $54.67 \pm 14.49$ & $49.13 \pm 2.93$ \\
\hline Threshold (mV) & $-18.17 \pm 7.68$ & $-7.44 \pm 0.56 * *$ \\
\hline Duration (ms) & $4.01 \pm 1.66$ & $4.74 \pm 0.49$ \\
\hline AHP Trough (mV) & $-7.84 \pm 5.92$ & $-8.31 \pm 1.20$ \\
\hline Peak IF (Hz) & $39.63 \pm 19.04$ & $39.81 \pm 2.91$ \\
\hline
\end{tabular}



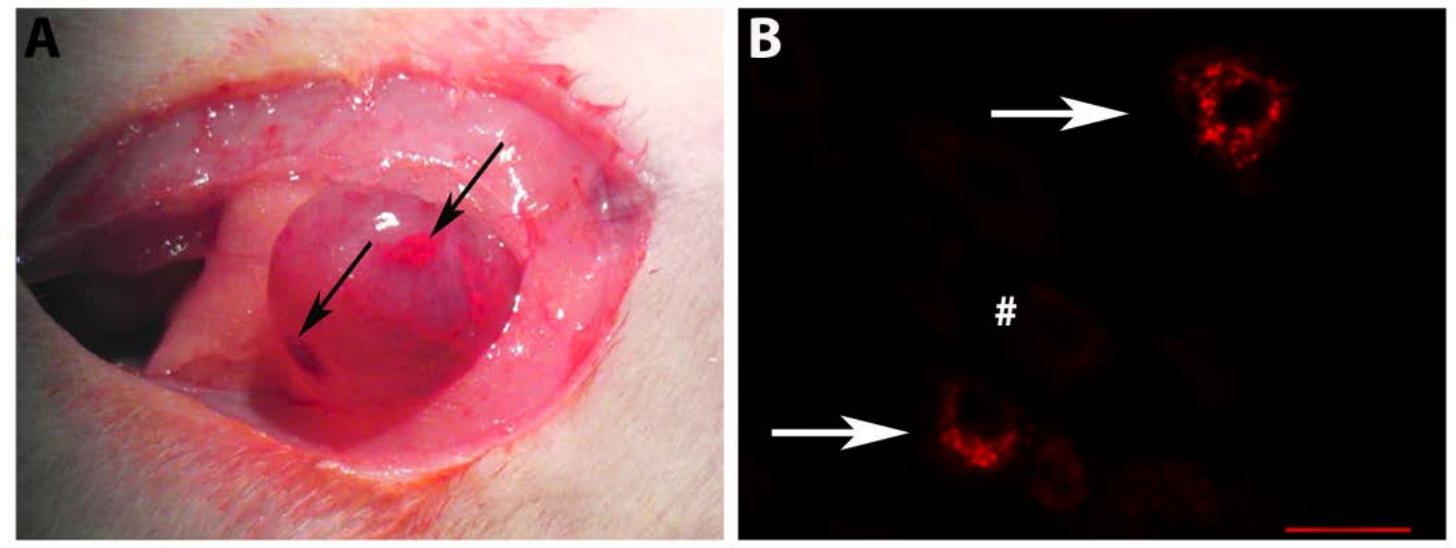

Figure 16 Retrograde tracer injections and identification of bladder NG neurons

The retrograde tracer Fast Dil was injected into the lining of the bladder (A, black arrows) 7-10 days prior to NG dissociation. A12 $\mu$ m thick section of the NG used for epifluorescence purposes reveals Dil+ neurons (B, white arrows) and Dil negative neurons (B, numeric sign). Scale bar indicates $25 \mu \mathrm{m}$. 


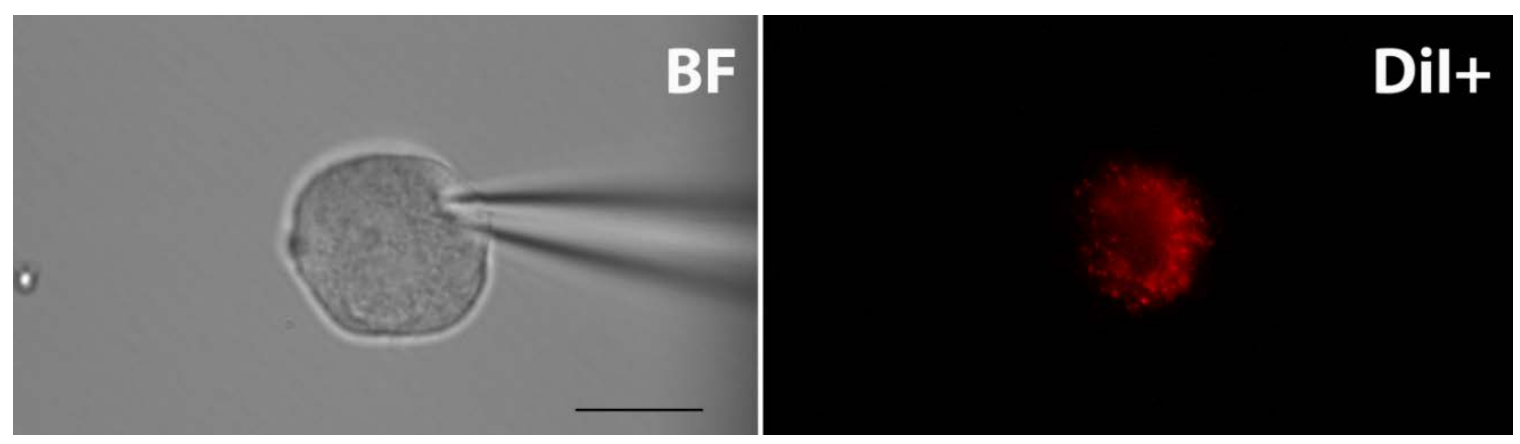

Figure 17 Whole cell patch-clamp electrophysiological recording of an NG bladder afferent

The Brightfield (BF) image illustrates the recording electrode forming a $G \Omega$ seal on a NG neuron that also is Dil+. Images were captured at 40X magnification. Scale bar indicates $25 \mu \mathrm{m}$. 


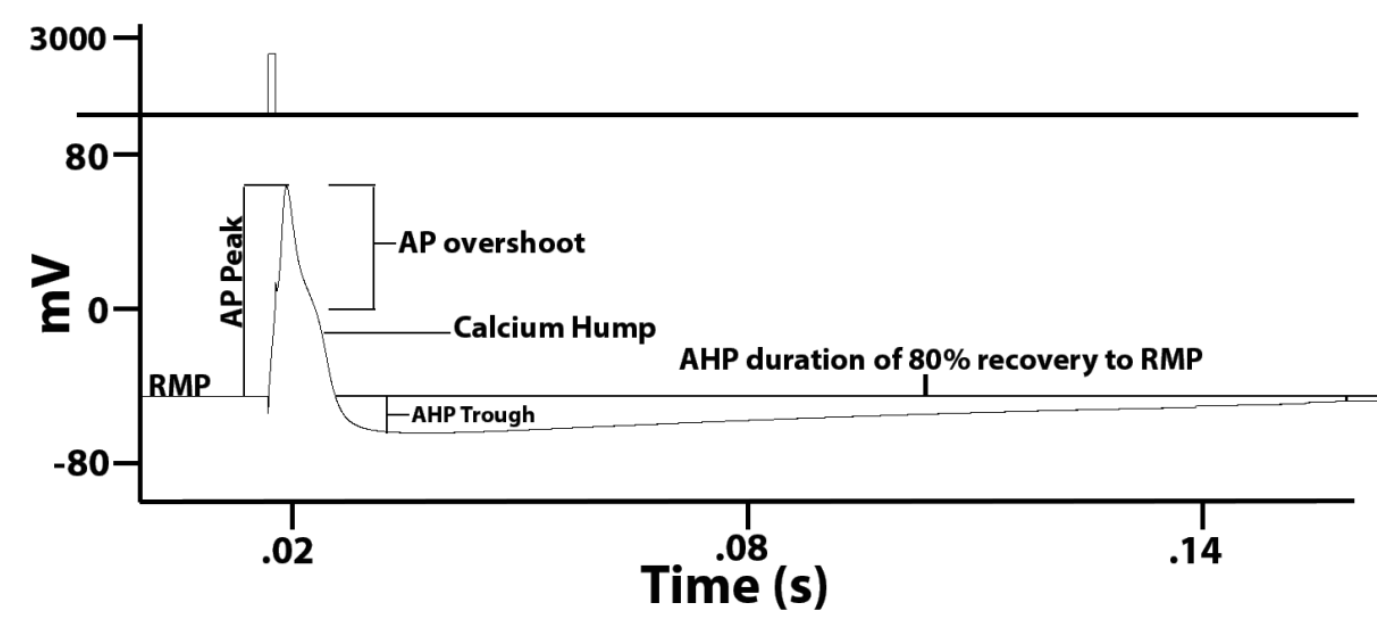

Figure 18 AP characteristics of a NG neuron innervating the bladder

An example of the action potential characteristics of a Dil+ NG neuron following a $2500 p A$ current injection. Note the presence of the calcium hump on the repolarization phase of the AP, typical of C-type (unmyelinated) NG neurons. 


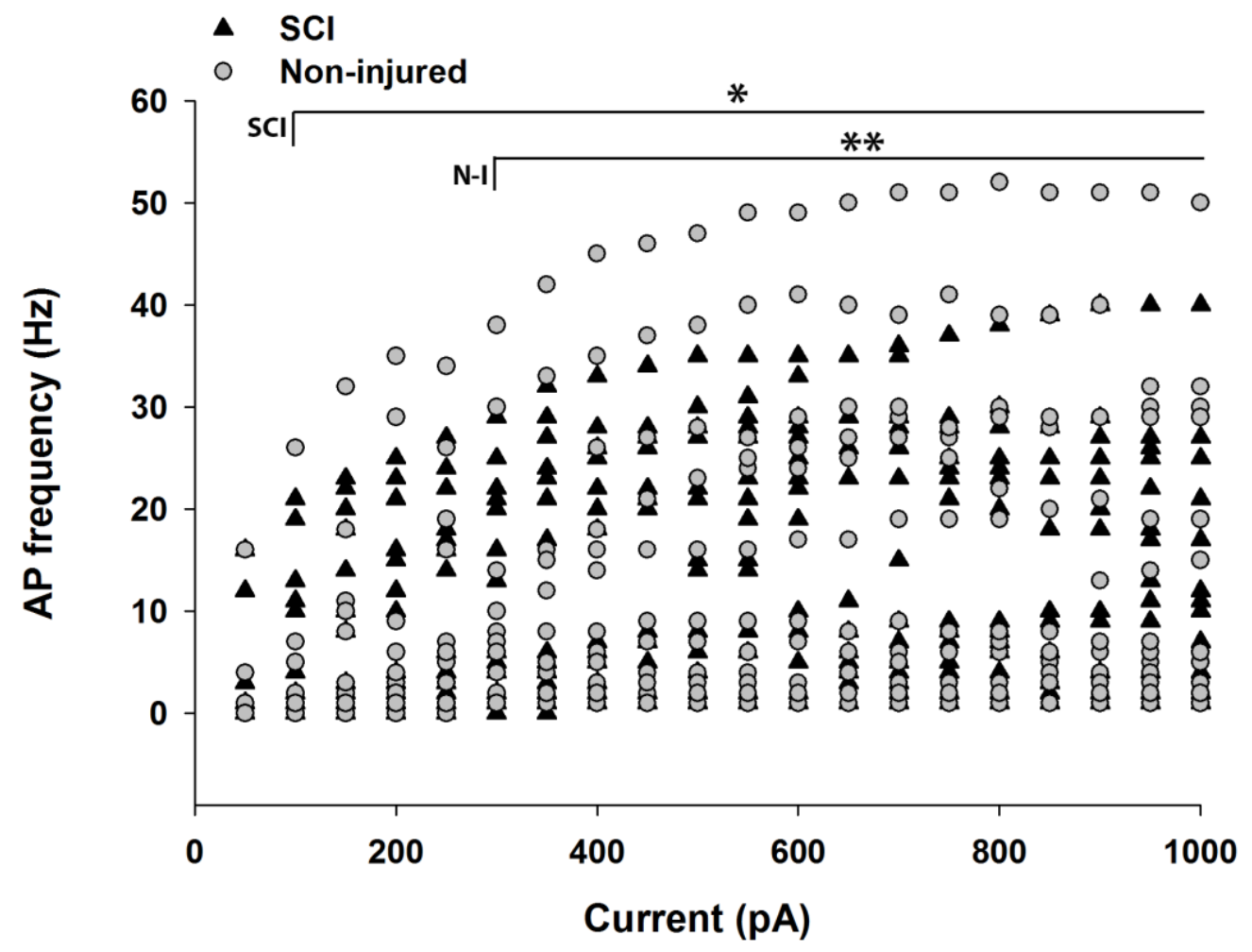

Figure 19 Distribution of non-injured and $\mathrm{SCI}$ high and low responders

The number of APs quantified for each cell and each group was separated based on the grand median value of 6 APs and plotted for each step of current injection. Non-injured cells represented a significant distribution of high to low responders from 300pA to 950pA, $p<.001$ and at 1000pA, $p<.005$. SCl cells represented a significant separation into distinct subsets of high to low responders from 100pA$1000 p A(p<.05$ at 100pA, 900pA, 950pA, 1000pA; $p<.01$ at $850 p A ; p<.005$ at 100pA, 400pA, 700pA, 750pA, 800pA; $p=.001$ at 200pA, 250pA, 550pA; $p<.001$ at 300pA, 350pA, 450pA, 500pA, 600pA, 650pA) (Median test between responders, grand median of $6, \mathrm{n}=33$ total cells). 


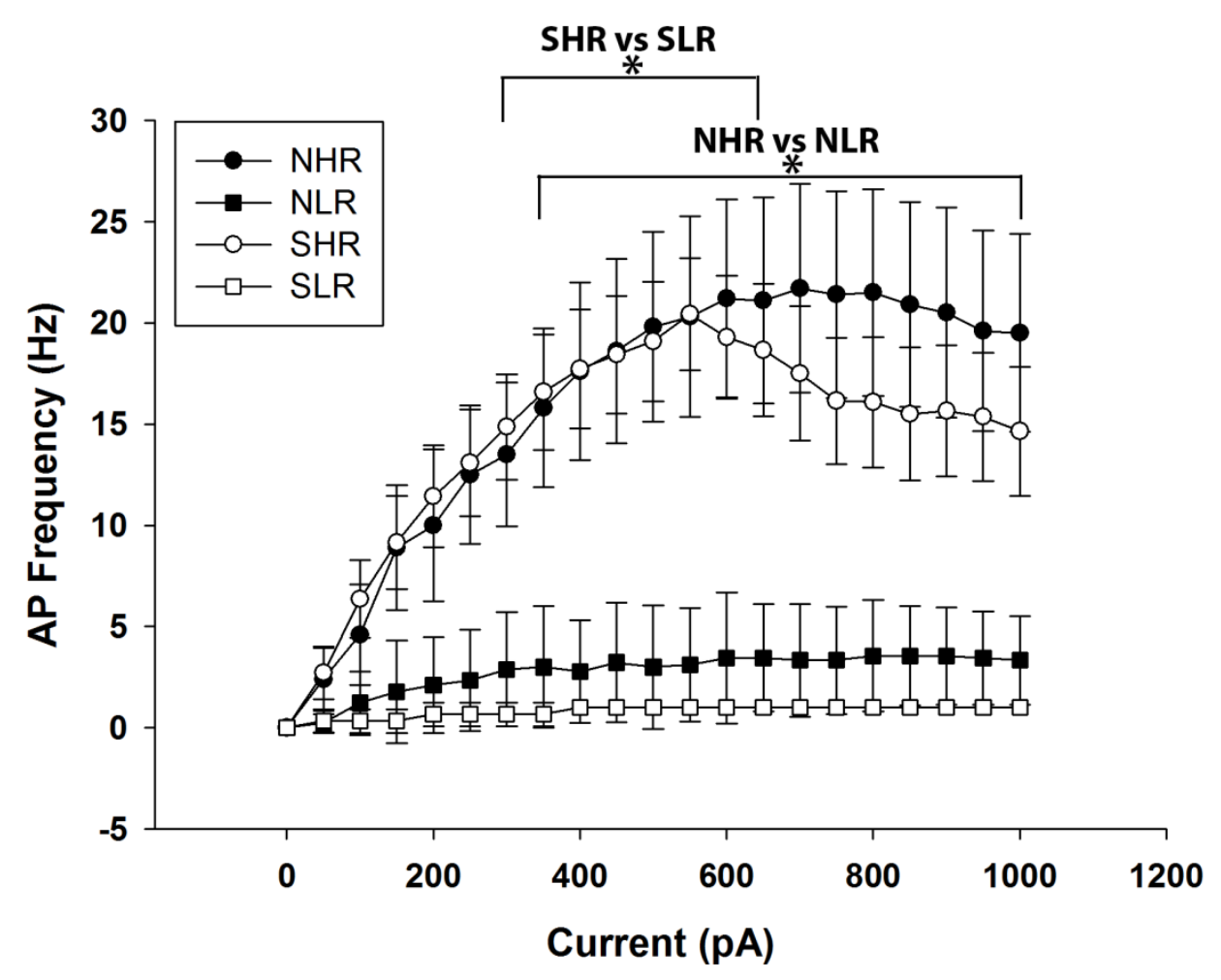

Figure 20 Current-Frequency plots of high and low responders in both the intact and SCl populations of bladder-innervating NG neurons.

Current frequency curves of all subsets of Dil+ bladder-innervating NG neurons are plotted. Non-injured high responders (NHR) maintained greater AP frequency from (350pA-1000pA, $\left.{ }^{*} \mathrm{p}<.05\right)$ compared to the non-injured low responders (NLR). SCl high responder (SHR) AP frequency was significantly greater than $\mathrm{SCl}$ low responders (SLR) from (300pA-650pA, $\left.{ }^{*}<<.05\right)$, after which AP frequency declines. (RM mixed model ANOVA with Tukey HSD post hoc t-test, $\mathrm{n}=33$ cells, mean $\pm \mathrm{SD}$ ) 


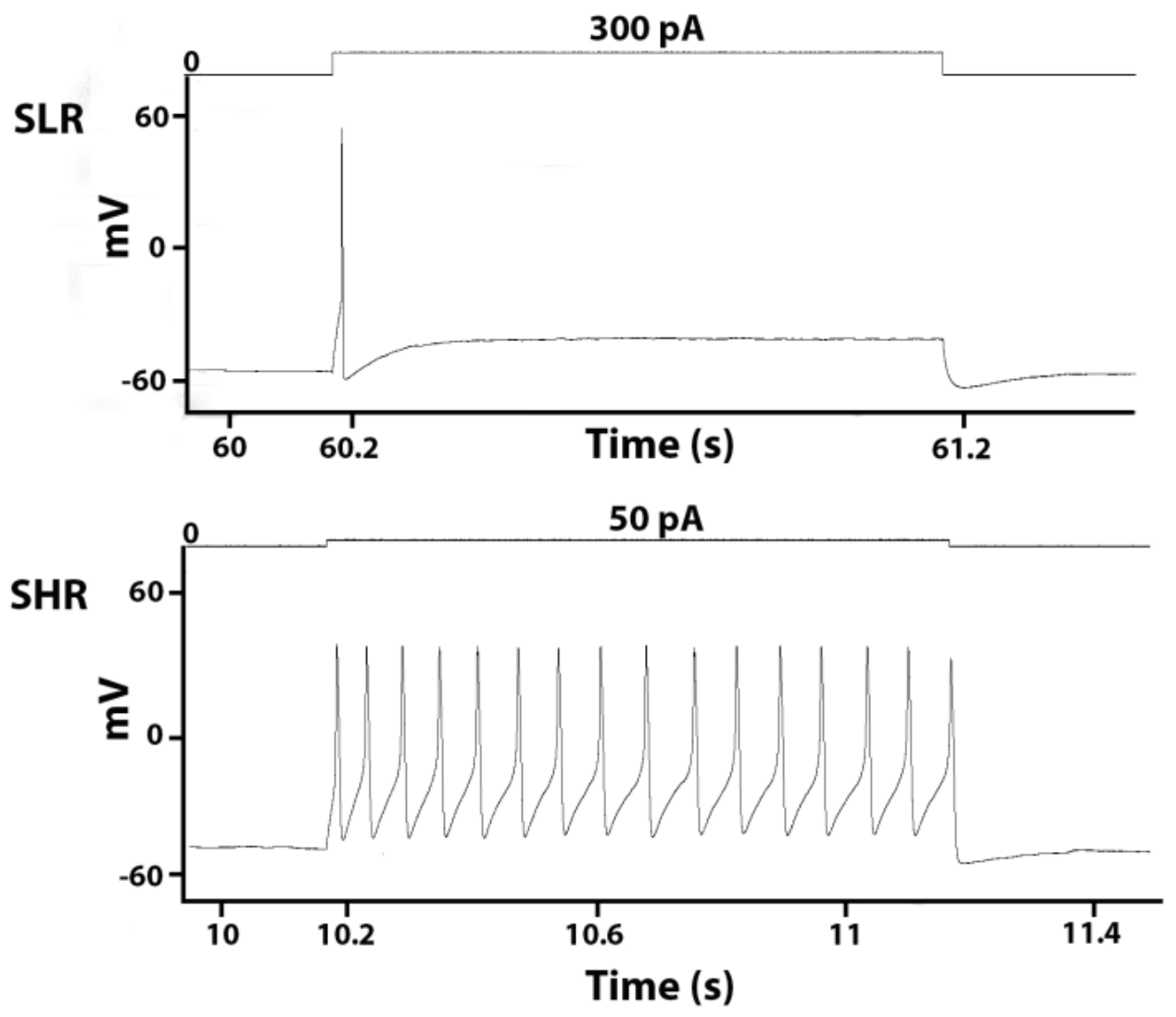

Figure 21 AP profile of $\mathrm{SCl}$ high and low responders

The top trace illustrates an example of a $\mathrm{SCl}$ low responder (SLR), firing a single action potential at a higher than average rheobase (300pA). The bottom trace is an example of an $\mathrm{SCl}$ high responder (SHR), firing multiple action potentials at a lower than average rheobase (50pA). 


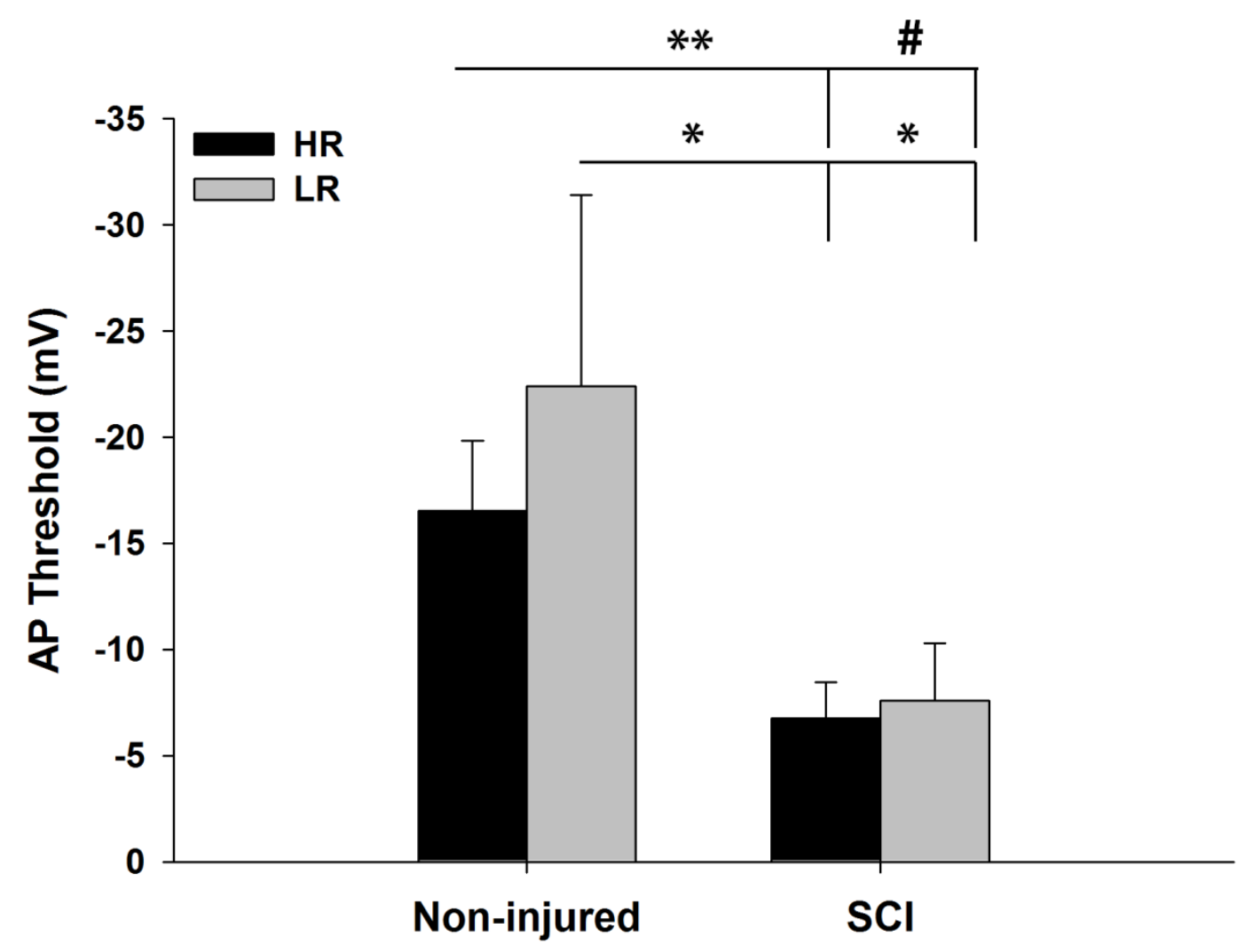

Figure 22 The effect of SCl on AP threshold in NG Dil+ neurons

A grouped bar graph illustrating that SCI resulted in a significantly greater (less negative) depolarizing threshold in both the HR and LR subsets compared to the non-injured subsets (SCI HR vs Non-injured HR, ${ }^{\star \star} p=.001$; SCl HR vs Noninjured LR, ${ }^{\star} p<.001 ;$ SCI LR vs Non-injured LR, ${ }^{\star} p<.001 ; S C I$ LR vs Non-injured HR, \#p<.05) (One-way ANOVA with Tukey HSD post hoc t-tests, $n=33$ cells, mean $\pm S D)$. 


\section{CHAPTER V}

\section{GENERAL DISCUSSION AND FUTURE DIRECTIONS}

This dissertation aimed to develop a thorough understanding of the neural innervation to the bladder following chronic $\mathrm{SCl}$ by examining the vagus nerve, a route that bypasses the spinal cord injury ( $\mathrm{SCl}$ ) in an effort to improve post-SCl bladder management and visceral pain syndromes. Apart from the paralysis that ensues, major complications of $\mathrm{SCl}$ also include deficits to bladder, bowel and sexual function as well as autonomic function and chronic pain. The vagus nerve, having cell bodies located in the nodose ganglia (NG) and terminals in the solitary nucleus is a route through which information from regions below the level of spinal injury can travel directly to the brainstem, by-passing the spinal cord. The extent of this extraspinal route has important implications in regards to understanding many of the post-SCI visceral symptoms and perceptual responses evident in clinically complete SCI patients (Wyndaele, 1991, Komisaruk et al., 1997, Ersoz and Akyuz, 2004, Komisaruk et al., 2004). . The vagus nerve may serve as an important source of input to the brain as the majority of visceral organs receive a dual sensory innervation from both the vagus as well as spinal nerves (Burden et al., 1983, Gattone et al., 1986, Jancso and Maggi, 1987, Neuhuber, 1987, Springall et al., 1987, Altschuler et al., 1989, Neuhuber, 1989, Ortega-Villalobos et al., 1990, Berthoud and Powley, 1992, 
Kummer et al., 1992, Altschuler et al., 1993, Cheng et al., 1997, Komisaruk et al., 1997, Collins et al., 1999, Vizzard et al., 2000, Hubscher et al., 2004, Komisaruk et al., 2004, Herrity et al., 2014). The vagus nerve is only thought to innervate thoraco-abdominal viscera as far caudally as the transverse colon in humans and is initially supported by the finding that electrical stimulation of the vagus nerve in the spinally intact dog has no effect on bladder pressure (Rozman and Bunc, 2004). However, in humans, functionally complete SCI patients have reported significant analgesia in response to self-stimulation to the genital area and some even were aware of orgasms (Komisaruk et al., 1997). Functional magnetic resonance imaging also demonstrated activation of the solitary nucleus (terminal site of the vagus) upon cervical self-stimulation in functionally complete SCI women (Komisaruk et al., 2004).

Although anatomical evidence of connections between the pelvic viscera and vagus nerve is discussed in the clinical literature pertaining to chronic SCl cases, no human studies on non-injured individuals demonstrate a vagal connection to the bladder, suggesting the possibility of reorganization of the circuitry under pathological conditions. It is possible that the neural connections are infrequently used in the normal condition and become revealed only with pathology, which is perhaps why it has not been seen in humans. For instance, in the urinary system after $\mathrm{SCl}$, descending control from higher centers in the midbrain is severed, triggering a local spinal segmental voiding reflex, driven by an "exposed" C-fiber circuitry. In the non-injured state, C-fibers innervating the 
bladder normally relay responses to noxious stimuli and have a limited role in normal voiding ("silent") (Habler et al., 1990). After SCl, it appears that these fibers now become sensitive not only to noxious stimuli, but also normal bladder filling and can play a role in hyperreflexia of the bladder detrusor after injury (de Groat, 1995). It is highly unlikely that the vagus nerve develops new major peripheral projections in pathological conditions, suggesting that these connections are likely there since development, but are perhaps "masked" and have not been observed. It is not yet known if vagal afferents innervating the bladder contribute to post-SCI bladder dysfunction. However, support of an extraspinal pathway involved in the neural control of the lower urinary tract stems from promising preliminary work from Ruggieri et al. They demonstrate significant activation of brain regions receiving vagal inputs (solitary nucleus, NTS) during saline bladder filling using functional magnetic resonance imaging in clinically complete SCI patients (Krhut J, 2014).

Once the anatomical innervation from the vagus nerve to the bladder was identified in this project, we next sought to characterize these afferents by examining their immunohistochemical phenotype and evaluating how $\mathrm{SCl}$ might alter that phenotype. Information gained from these experiments may help provide insight into particular sensory processes bladder-innervating vagal afferents play a role in. For example, one important molecular marker that was focused on, P2X3 plays an essential role in bladder sensory transduction. We found that $\mathrm{SCl}$ results in a significant increase in the number of NG neurons 
expressing this particular marker, and this result also was present in the majority of the bladder-innervating NG neurons, suggesting vagal afferents may play a role in the sensory portion of the micturition reflex (Chapter III). A future direction would be to examine peripheral vagal afferent terminal innervation into different layers of the bladder and how this contribution from the vagus nerve is altered after $\mathrm{SCl}$.

Identification of the extent of vagal afferent innervation to the bladder becomes particularly important especially since the first step in initiating the micturition reflex involves the relationship between afferent fibers and urothelial/suburothelial tissue layers participating in bladder distension. It is also shown that sensory afferents innervating the bladder can release neurotransmitters and neuropeptides from their peripheral terminals, which can act in an efferent manner by influencing smooth muscle activity, triggering local inflammatory responses and participating in autonomic reflexes (de Groat, 1987a). Immunofluorescence studies of spinal innervation in both the rat and human bladder show numerous CGRP- and SP-containing free nerve terminal endings in the submucosal and urothelial layers (Yokokawa et al., 1986, Wakabayashi et al., 1993). Electron microscopy studies also have provided evidence of dense networks containing nerve fibers and capillaries between the urothelium and lamina propria (Inoue and Gabella, 1992). With the majority of fibers being unmyelinated (Zhuo et al., 1997), it is anticipated that parasympathetic vagal afferents innervating the bladder would share a similar 
layer distribution as spinal parasympathetic pelvic nerve afferents (C-fibers portion), having a widespread distribution in the detrusor and urothelium (Kanai and Andersson, 2010). This position would allow them to detect volume changes from urothelial cells as well as stretch and tension in response to bladder distention. Vagal afferents are already primed to do so as they have specialized nerve endings termed IMAs (intramuscular arrays) and IGLEs (intraganglionic laminar endings) that are thought to function like pacinian or golgi-tendon organs, sensing stretch and tension/contraction in the stomach and gut wall (Phillips and Powley, 2000).

Chapter III of this dissertation discussed how activation of the P2X3 receptor, which is predominantly expressed on sensory afferents (Chen et al., 1995, Lewis et al., 1995) including NG neurons (Lewis et al., 1995, Hubscher et al., 2001, Dang et al., 2005, Kestler et al., 2009) and in NG neurons innervating the bladder (Herrity et al., 2014), has been established as one of the peripheral receptors facilitating afferent transmission of the micturition reflex (Cockayne et al., 2000, King et al., 2004). Immunoreactivity to the P2X3 receptor has been found on pelvic afferent terminal fibers in the mouse (Vlaskovska et al., 2001) as well as in human bladder urothelial tissue (Elneil et al., 2001, Yiangou et al., 2001). In other tissue layers of the bladder such as the detrusor muscle, activated P2X3 enhances smooth muscle contraction especially in conditions of overactivity or inflammation when the P2X3 ligand, ATP, appears to be in excess (Hoyle, 1994, Palea et al., 1995, D'Agostino et al., 2012). 
$\mathrm{P} 2 \mathrm{X} 3$ receptors also are present on cells located in the suburothelial layer (myofibroblasts/interstitial cells) which have been proposed to play a role in the sensation of bladder fullness (Wiseman et al., 2003, Sui et al., 2004). ATPsensitive afferent fibers may therefore be well suited to signal the degree of bladder distention. Based on the results from Chapter 3 of this dissertation, it appears that vagal afferents innervating the bladder also have the potential to signal through purinergic mechanisms via P2X3 receptors. Further evaluation of P2X3-immunoreactive vagal afferents innervating the bladder may be an important endeavor since the presence of this receptor is evident in multiple bladder tissue layers, it is involved in regulating both afferent and efferent pathways of the bladder circuitry (Ford et al., 2006) and it plays a role in disease states such as overactive bladder syndrome, neurogenic bladder and interstitial cystitis (Burnstock, 2014). It should be noted however, that the control of micturition actually involves many neurotransmitters, neuropeptides and different receptors and ion channels, all of which could be potential pharmacological targets for therapeutic interventions (de Groat and Yoshimura, 2001). However, the challenge is to identify drugs that exhibit a selective action on the lower urinary tract without the addition of undesirable side effects that could potentially alter normal bladder function. A combinational approach to treating bladder dysfunction may be more advantageous.

Additional future directions for this project would be to examine the degree of overlap with the bladder from multiple visceral organs in the NG (primary 
afferent level) as well as the central inputs and possible viscero-visceral convergence from the vagus nerve in the solitary nucleus (secondary afferent level). In general, cross-talk between pelvic organ systems, such as with the bladder and colon, exist to aide in normal visceral functioning and are usually cross-inhibitory reflexes (i.e. voiding and defecation occur alternatively, voiding takes precedence over defecation) (Basinski et al., 2003). The vagus nerve also participates in regulatory cross-talk as evidenced with the baroreceptor reflex, demonstrating its role in maintaining stable blood pressure and heart rate (Dampney, 1994, Spyer, 1994). Under pathological conditions, cross-excitatory reflexes occur when a disease state in one organ influences the functionality of an adjacent, sometimes distant organ (Qin et al., 2007, Kaddumi et al., 2012) through shared (i.e. dichotomizing, trichotomizing) pathways. Cross-sensitization between the bladder and other pelvic viscera is a clinical concern that makes diagnosing and treating the etiology difficult (Berkley, 2005, Berkley et al., 2005, Baranowski et al., 2008, Theoharides et al., 2008, van de Merwe et al., 2008). In humans and in animals, the organs that seem to be most often involved in pelvic cross-sensitization besides bladder and colon, with respect to spinal afferents, appear to be the pelvic urethra and the uterus (Dmitrieva et al., 2001, Giamberardino et al., 2001, Dmitrieva and Berkley, 2002, Morrison et al., 2006, Winnard et al., 2006, Peng et al., 2008a, Peng et al., 2008b, Peng et al., 2009). The extent of viscero-visceral interaction between these organs and the degree of overlap with the bladder in the NG has not been established yet. 
Unpublished preliminary data from (Ruggieri, 2014) suggests that vagal afferents do not innervate the pelvic urethra, as fast blue labeled cell bodies traced from this organ were not observed in the female canine NG or NTS. However, it is unclear whether or not a specific area of the urethra (external or internal urethral sphincter [EUS, IUS, respectively]) was targeted with the tracer injection in this instance. A follow-up tracing study between male and female rats would be beneficial since sex differences exist with respect to the function of these sphincters. The female internal urethral smooth muscle sphincter is not as distinct at the bladder neck as it is in males, which serves important role in preventing reflux of ejaculate into the bladder (Shafik, 1997). The functional relevance of the IUS in the female is unclear as continence does not appear to be affected by an incompetent bladder neck (Versi et al., 1986). On the other hand, the EUS is more intricate in females as it serves to contract both the urethra and vagina (Oelrich, 1983). The relationship of the urethra with the vagina also is functionally important for assisting the muscles of the pelvic floor after a vaginal delivery (Phillips and Monga, 2005). Since voluntary control of voiding is possible only if neural innervation to both the bladder and urethra is intact (Elbadawi and Schenk, 1974), examining the potential vagal contribution to the urethral sphincters and the degree of overlap with the bladder in the NG may suggest further evidence of a possible functional relationship between the two structures, which are essential for the storage and elimination of urine. 
Additional important pelvic regulatory processes, such as micturition, defecation, and ejaculation, are spinobulbospinal reflexes and possible viscerovisceral interactions could be due to the convergence of visceral inputs that occur not only through peripherally mediated mechanisms, but also due to convergent input onto second order supraspinal neurons, such as the NTS and medullary reticular formation (Berkley et al., 2005, Qin et al., 2005, Kaddumi and Hubscher, 2006, Brumovsky and Gebhart, 2010). Promising new data from Ruggieri et al. assessing bladder re-innervation strategies in a lower motoneuron $\mathrm{SCl}$ canine model, found evidence of dye labeled cells in both the NG and NTS following fluorogold retrograde tracer injection in the bladder wall. The use of fluorphoreconjugated dextran amines as a follow-up step should result in robust labeling at the secondary afferent level in the NTS. Although tracing data from us and that of others has revealed a homogenous distribution of visceral afferent labeling in nodose ganglion neurons (Sharkey and Williams, 1983, Altschuler et al., 1989, Zhong et al., 2008, Herrity et al., 2014), we would expect to find a viscerotopic organization of terminal labeling in the NTS. For instance, (Altschuler et al., 1993) showed that following tracer injection of CT-HRP into the colon, labeling in the NTS was localized in the dorsal region of the commissural NTS, and (Hubscher and Berkley, 1994) have shown that whereas neurons in the rostral NTS process gustatory information, neurons in the caudal NTS receive inputs from pelvic reproductive organs (vagina, cervix, uterine horn and colon). A 
specific organized distribution may have important implications for the coordination of visceral processing.

It has been reported that sensory neurons in the NTS project to the parabrachial nucleus and that information can then be relayed to the amygdala, hypothalamus and limbic cortex which may influence the autonomic and emotional responses to noxious visceral stimuli (Cechetto, 1995). Using a model of noxious gastric distention, research from Traub and colleagues also suggest that while vagal afferents can participate in the processing of noxious visceral stimuli, it is likely that they contribute to the affective-emotional aspect of pain versus the sensory-discriminative aspect (Traub et al., 1996). Furthermore, vagal afferents projecting from the lungs to the NTS and then to the parabrachial pontine region have been suggested to contribute to plasticity involved in respiratory control as well as influencing the integration of other systems involved in that control, including voluntary breathing, sleep-wake patterns, and emotions (Kubin et al., 2006). Analysis of higher order neuronal levels, such as with the use of fMRI, during measures of bladder capacity and output following chronic $\mathrm{SCl}$ may point to areas in the brain associated with vagal inputs that could play a role in the micturition reflex after injury (Krhut J, 2014).

In addition to identifying the extent of vagal innervation to the bladder and characterizing those neurons using immunohistochemistry, we also examined the electrophysiological properties of the NG neurons innervating the bladder and how those properties were effected by $\mathrm{SCl}$. Overall, $\mathrm{SCl}$ resulted in increased 
excitability in the bladder-innervating population of NG neurons as demonstrated by a significant overall decrease in threshold for eliciting an AP as well as a significant increase in membrane resistance. A broader aspect to consider is whether the alterations in the bladder-innervating NG neuronal membrane properties are modulated by disease states and are important, in this case, for the system's response to $\mathrm{SCl}$, perhaps initiating some plasticity-related changes in those fibers. Future work will include immunocytochemistry on preserved Dil+ NG recorded cells (bladder-innervating) from the electrophysiological experiments to assess differential expression of activating transcription factor (ATF-3), a sensitive marker of nerve injury (Tsujino et al., 2000) and cellular stress/damage (Hai et al., 1999, Allen-Jennings et al., 2001, Okamoto et al., 2001, Kawauchi et al., 2002, Hartman et al., 2004). This staining will be performed in both the non-injured and $\mathrm{SCl}$ populations in order to determine if our procedures (tracer injection, tracer incorporation, dissociation) resulted in an injury or stress response that could potentially alter the electrophysiological properties of those neurons (Rau et al., 2014). As a control to aide in this understanding, staining for ATF3 will be performed on NG sections taken from a rat receiving injection of bladder tracer, but perfused prior to ganglion excision. At the same time point, additional immunocytochemical analysis will include an assessment of P2X3 expression in the Dil+ NG neurons. We would expect to find that the Dil+ NG cells from the SCI group would exhibit a greater proportion of 
P2X3 expressing neurons relative to the non-injured group, which would parallel our findings in Chapter III.

Examining the electrophysiological properties in bladder-innervating NG neurons helps provide an understanding of the ways in which excitability following $\mathrm{SCl}$ could correlate with functional measures (cystometrogram) of bladder storage and output. Alterations to the bladder tissue itself after $\mathrm{SCl}$ has the potential to impact AP discharge patterns. Measures of vagal afferent action after SCI could display a phasic pattern of activity, characteristic of spinal bladder afferents mediating the switch-like manner of bladder operation: storage and elimination (de Groat, 1995). On the other hand, it is possible that NG bladder afferents may behave in a tonic mode, reflecting the nature of autonomic function to the respiratory and cardiovascular system for example (McAlexander et al., 1999).

With the ultimate goal of targeting this extraspinal pathway for translational objectives, future studies may involve the identification of suitable biomarkers in the bladder that could serve as a way to define the presence of or measure the degree of clinical bladder pathology and the physiological response to a treatment (vagal nerve stimulation). Vagus nerve stimulation (VNS) has been approved by the Food and Drug Administration for the treatment of refractory epilepsy and depression and more recently, is being studied for its applications in obesity, Alzheimer's disease, heart failure, and inflammatory disease (Tracey, 2002, Li et al., 2004, Groves and Brown, 2005, Milby et al., 2008, Schwartz et al., 
2008). Given that the vagus nerve would not be distinguishable from sympathetic fibers once it enters the abdominal region (Shoja M., 2013), the most common area of access to the nerve for stimulation would be on the cervical vagus. Typically, the left cervical vagus is used for therapeutic stimulation since it does not affect heart rate (Saper et al., 1990, Schachter and Saper, 1998). Despite differential roles of the vagus nerve to cardiac function (Randall et al., 1986, Saper et al., 1990, Schachter and Saper, 1998), we did not find any differences in bladder NG labeling between the right and left side (Herrity et al., 2014). Treatment stimulation parameters have been variable due to the fact that the vagus nerve is primarily comprised of small diameter $(0.2-2 \mu \mathrm{m})$, unmyelinated cfibers with a slow conduction velocity $(0.3-2 \mathrm{~m} / \mathrm{s})$ and properly accessing this population of afferents requires a higher stimulation waveform and longer duration (Castoro et al., 2011). As a result, unwanted side effects such as hoarseness, neck pain, coughing, difficulty breathing and swallowing have been reported (Milby et al., 2008). VNS causes antidromic and orthodromic activation of nerve fibers and the challenge is trying to achieve a balance between therapeutic benefit while mitigating unwanted side-effects. Since every patient presents with a unique set of symptoms related to their condition, clinicians should have the capability to alter electrode frequency and waveforms to best suit the patients' condition.

To investigate the influence of VNS therapy on the bladder, one potential avenue would be to assess urinary biomarkers, as urine samples from patients 
would be easy to collect. Another possibility may include a physical measurement, such as bladder wall thickness, which is measured via transabdominal ultrasound (Fry et al., 2014). Given its role in the regulation of bladder function, urinary ATP may be considered a potential biomarker. Collection is non-invasive and serves as a reliable measurement method (luciferin-luciferase bioluminescence assay). Care must be taken to immediately snap freeze samples after collection in order to avoid ATP degradation (SilvaRamos et al., 2013). ATP release is enhanced in patients with overactive bladder and interstitial cystitis and excesses of ATP are thought to underlie the enhanced sensation or bladder urgency (Burnstock, 2014).

Other popular candidates have included the presence of inflammatory mediators such as cytokines and prostaglandins as well as nerve growth factor (NGF). They have been shown to correlate with the severity of overactive bladder syndrome for example, but have not been extremely effective in terms of serving as an independent predictive measurement (Cartwright et al., 2011). In particular, excretion of NGF into the urine is increased in patients with overactive bladder syndrome and interstitial cystitis. However, its concentration has been reported to have wide variation in patients with overactive bladders and may not be sensitive enough to identify individuals with true pathology (Kuo et al., 2010, Liu et al., 2010, Ochodnicky et al., 2011). Other factors confounding its specificity for bladder dysfunction include a lack of a clear baseline measure of NGF in normal subjects as well as biochemical issues, such as NGF binding to cellular 
components and proteins present in the urine, thus not being able to serve as a clear indicator or measure of dysfunction (Ochodnicky et al., 2011).

Despite the fact that NGF may not serve as a reliable biomarker for bladder dysfunction, it still may be a viable marker to target therapeutically given its role in target organ-neural interactions as well as its response to locomotor step-training therapy (decrease in mRNA) and exercise that could potentially ameliorate bladder dysfunction post-SCI (Vizzard, 2000a, Steers and Tuttle, 2006, Vizzard, 2006, Liu et al., 2010, Ward et al., 2014). Subsequent detrusorsphincter dyssynergia that results after injury leads to a functionally obstructed urethra and eventually, bladder hypertrophy. In response to these events, NGF is released in the bladder and transported to bladder afferent pathways leading to hyperexcitability of these fibers and ultimately influencing neurogenic detrusor over activity. NGF can function to sensitize the C-fiber bladder afferents and change their responses to mechanical stimuli, making them more excitable (de Groat and Yoshimura, 2006, 2010).

In addition to its release in the bladder, NGF also is increased in the spinal cord after SCl, contributing to hyperexcitability of the bladder afferent pathways (Yoshimura, 1999, Seki et al., 2002). In SCl rats, direct targeting of NGF with intrathecal administration of NGF antibodies, led to a reduction in NGF levels at the L6 spinal cord and resulted in a suppression of detrusor hyperreflexia and improved bladder-urethral function (Seki et al., 2002, Seki et al., 2004). In a phase two clinical trial, Tanezumab, a humanized anti-NGF monoclonal antibody 
that binds with high affinity to NGF preventing it from interacting with TrkA receptors on nociceptive neurons, was tested for efficacy in patients with moderate to severe interstitial cystitis associated pain (Evans et al., 2011). Although Tanezumab decreased urinary frequency, some patients experienced paresthesia, hypoesthesia and arthralgia due to a systemic type-blockade of NGF at sites other than the bladder (i.e. peripheral joints) (Evans et al., 2011, Kashyap et al., 2013). In an attempt to limit these global side-effects, novel intravesical treatments targeting urinary NGF have been more recently explored using antisense oligonucleotides complexed to liposomes (Kashyap et al., 2013). This approach may be more advantageous to treating altered NGF levels since the afferent nerve fibers that play a large role in the development of bladder overactivity and/or neurogenic bladder form a dense network near the urothelium, thus readily accessible to this type of treatment. Overall, it highlights the dynamic relationship between target organs and their neural connections and how the two influence each other in terms of contributing to normal physiological homeostasis to triggering pathology and developing treatment strategies that address both structures.

Overall, this project identifies the anatomical innervation of the bladder by sensory vagal afferents (Figure 23), which by-pass the spinal cord, and confirms the vagal anatomical connection with the distal colon. A pre-existing neural substrate through vagal afferents can mediate physiological reflexes between the bladder and colon that are important for maintaining homeostasis. In addition, 
through dichotomizing vagal afferent pathways and cross sensitization mechanisms, pathology in either the bladder or colon could contribute to the clinical overlap of conditions such as interstitial cystitis or irritable bowel syndrome. Even though this project points to the significant role dichotomizing afferents play with respect to cross-talk between two visceral organs, NG neurons could display multi-organ innervation given the extent of its contribution to numerous viscera. We also demonstrated the vagus nerve provides a substantial degree of innervation to the bladder, which was equivalent to the parasympathetic spinal supply.

These identified vagal pathways to the bladder and colon provide an extraspinal route through which sensory information from below the level of a spinal injury can flow to the brainstem. Despite the fact that $\mathrm{SCl}$ does not directly damage the vagus nerve, we demonstrate that vagal afferents are indirectly affected by a distant, chronic spinal injury and respond by modifying both their neurochemical and electrophysiological properties. Following SCI, NG neurons exhibit an immunohistochemical phenotypic shift, represented by a change in the proportion of neurons expressing different injury-responsive markers, which also held true for many bladder-innervating NG neurons. In addition, alterations in the electrophysiological membrane properties of bladder-innervating NG neurons suggest they also are sensitive to a remote spinal injury. Overall, characterizing vagal neurons, including those that innervate the bladder will provide further 
insight into their role in afferent signaling in the context where descending, tonic control to the bladder is removed. 


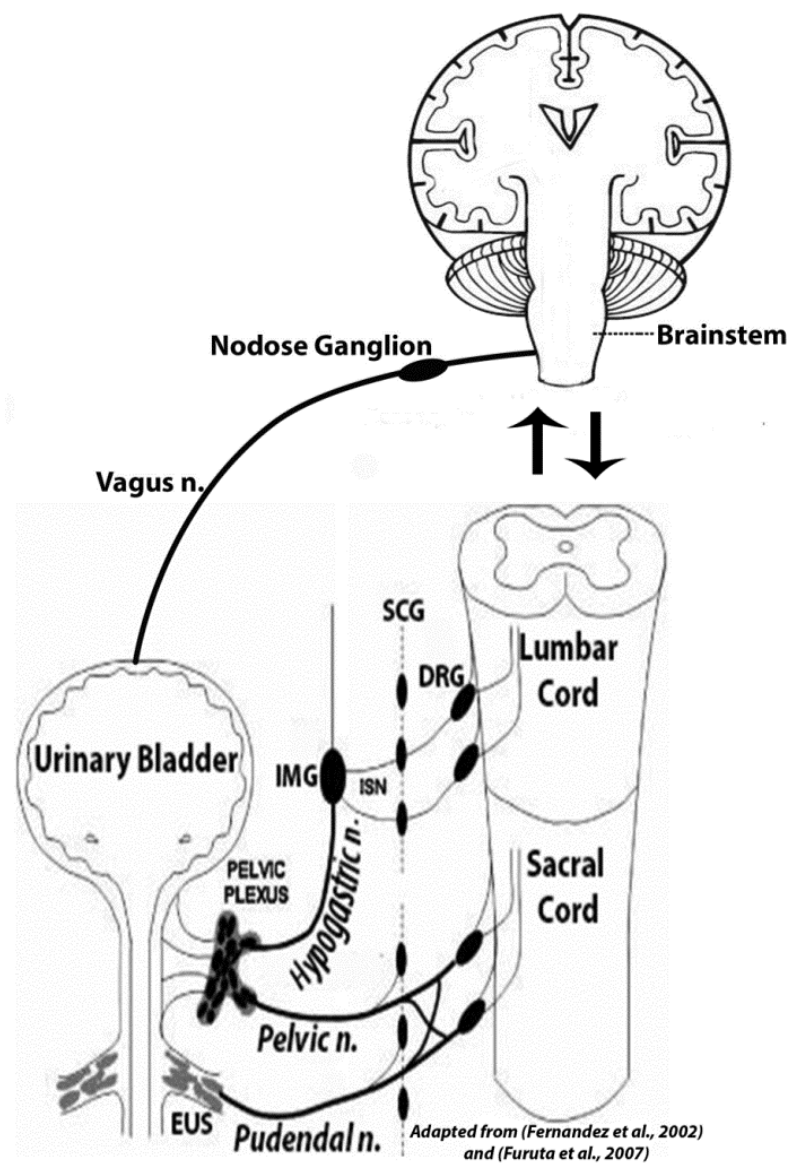

Figure 23 Anatomical innervation of the bladder by the vagus nerve

A summary figure is provided which demonstrates the dual sensory supply from both vagal and spinal sources to the urinary bladder. Adapted from (Fernandez, 2002, Furuta et al., 2007). Dorsal root ganglia (DRG), external urethral sphincter (EUS), inferior mesenteric ganglia (IMG), inferior splanchnic nerves (ISN), sympathetic chain ganglia (SCG). 


\section{REFERENCES}

Aguayo LG, White G (1992) Effects of nerve growth factor on TTX- and capsaicin-sensitivity in adult rat sensory neurons. Brain Res 570:61-67.

Agur A, Dalley A (2009) Grant's Atlas of Anatomy. Bethesda: Lippincott Williams \& Wilins.

AHCPR U (1996) US Agency for Healthcare Quality and Research. Hospital Inpatient Statistics.

Alagiri M, Chottiner S, Ratner V, Slade D, Hanno PM (1997) Interstitial cystitis: unexplained associations with other chronic disease and pain syndromes. Urology 49:52-57.

Allen-Jennings AE, Hartman MG, Kociba GJ, Hai T (2001) The roles of ATF3 in glucose homeostasis. A transgenic mouse model with liver dysfunction and defects in endocrine pancreas. J Biol Chem 276:29507-29514.

Altschuler SM (2001) Laryngeal and respiratory protective reflexes. Am J Med 111 Suppl 8A:90S$94 \mathrm{~S}$.

Altschuler SM, Bao XM, Bieger D, Hopkins DA, Miselis RR (1989) Viscerotopic representation of the upper alimentary tract in the rat: sensory ganglia and nuclei of the solitary and spinal trigeminal tracts. J Comp Neurol 283:248-268.

Altschuler SM, Escardo J, Lynn RB, Miselis RR (1993) The central organization of the vagus nerve innervating the colon of the rat. Gastroenterology 104:502-509.

Amir R, Devor M (2000) Functional cross-excitation between afferent A- and C-neurons in dorsal root ganglia. Neuroscience 95:189-195.

Anderson KD (2004) Targeting recovery: priorities of the spinal cord-injured population. J Neurotrauma 21:1371-1383.

Angelucci A, Sainsbury K (2006) Contribution of feedforward thalamic afferents and corticogeniculate feedback to the spatial summation area of macaque V1 and LGN. J Comp Neurol 498:330-351.

Apodaca G, Kiss S, Ruiz W, Meyers S, Zeidel M, Birder L (2003) Disruption of bladder epithelium barrier function after spinal cord injury. Am J Physiol Renal Physiol 284:F966-976.

Arms L, Vizzard MA (2011) Neuropeptides in lower urinary tract function. Handb Exp Pharmacol 395-423.

Avelino A, Cruz C, Nagy I, Cruz F (2002) Vanilloid receptor 1 expression in the rat urinary tract. Neuroscience 109:787-798.

Averill S, McMahon SB, Clary DO, Reichardt LF, Priestley JV (1995) Immunocytochemical localization of trkA receptors in chemically identified subgroups of adult rat sensory neurons. Eur J Neurosci 7:1484-1494.

Averill S, Michael GJ, Shortland PJ, Leavesley RC, King VR, Bradbury EJ, McMahon SB, Priestley JV (2004) NGF and GDNF ameliorate the increase in ATF3 expression which occurs in dorsal root ganglion cells in response to peripheral nerve injury. Eur J Neurosci 19:1437-1445.

Aviado DM, Guevara Aviado D (2001) The Bezold-Jarisch reflex. A historical perspective of cardiopulmonary reflexes. Ann N Y Acad Sci 940:48-58.

Banerjee B, Medda BK, Lazarova Z, Bansal N, Shaker R, Sengupta JN (2007) Effect of refluxinduced inflammation on transient receptor potential vanilloid one (TRPV1) expression in primary sensory neurons innervating the oesophagus of rats. Neurogastroenterol Motil 19:681-691. 
Banerjee B, Medda BK, Schmidt J, Zheng Y, Zhang Z, Shaker R, Sengupta JN (2009a) Altered expression of P2X3 in vagal and spinal afferents following esophagitis in rats. Histochem Cell Biol 132:585-597.

Banerjee B, Medda BK, Zheng Y, Miller H, Miranda A, Sengupta JN, Shaker R (2009b) Alterations in $\mathrm{N}$-methyl-D-aspartate receptor subunits in primary sensory neurons following acidinduced esophagitis in cats. Am J Physiol Gastrointest Liver Physiol 296:G66-77.

Bao F, Brown A, Dekaban GA, Omana V, Weaver LC (2011) CD11d integrin blockade reduces the systemic inflammatory response syndrome after spinal cord injury. Exp Neurol 231:272283.

Baranowski AP, Abrams P, Berger RE, Buffington CA, de CWAC, Hanno P, Loeser JD, Nickel JC, Wesselmann $U$ (2008) Urogenital pain--time to accept a new approach to phenotyping and, as a consequence, management. Eur Urol 53:33-36.

Bartholdi D, Schwab ME (1997) Expression of pro-inflammatory cytokine and chemokine mRNA upon experimental spinal cord injury in mouse: an in situ hybridization study. Eur J Neurosci 9:1422-1438.

Basinski C, Fuller E, Brizendine EJ, Benson JT (2003) Bladder-anal reflex. Neurourol Urodyn 22:683-686.

Basso DM, Beattie MS, Bresnahan JC (1995) A sensitive and reliable locomotor rating scale for open field testing in rats. J Neurotrauma 12:1-21.

Basso DM, Beattie MS, Bresnahan JC (1996) Graded histological and locomotor outcomes after spinal cord contusion using the NYU weight-drop device versus transection. Exp Neurol 139:244-256.

Beckel JM, Holstege G (2011a) Neuroanatomy of the lower urinary tract. Handb Exp Pharmacol 99-116.

Beckel JM, Holstege G (2011b) Neurophysiology of the lower urinary tract. Handb Exp Pharmacol 149-169.

Beckel JM, Holstege G (2014) The lower urinary tract. In: The rat nervous system (Paxinos, ed): Elsevier.

Beckstead RM, Norgren R (1979) An autoradiographic examination of the central distribution of the trigeminal, facial, glossopharyngeal, and vagal nerves in the monkey. J Comp Neurol 184:455-472.

Benevento BT, Sipski ML (2002) Neurogenic bladder, neurogenic bowel, and sexual dysfunction in people with spinal cord injury. Phys Ther 82:601-612.

Bennett DL, Michael GJ, Ramachandran N, Munson JB, Averill S, Yan Q, McMahon SB, Priestley JV (1998) A distinct subgroup of small DRG cells express GDNF receptor components and GDNF is protective for these neurons after nerve injury. J Neurosci 18:3059-3072.

Berkley KJ (2005) A life of pelvic pain. Physiol Behav 86:272-280.

Berkley KJ, Rapkin AJ, Papka RE (2005) The pains of endometriosis. Science 308:1587-1589.

Berthoud HR, Lynn PA, Blackshaw LA (2001) Vagal and spinal mechanosensors in the rat stomach and colon have multiple receptive fields. Am J Physiol Regul Integr Comp Physiol 280:R1371-1381.

Berthoud HR, Neuhuber WL (2000) Functional and chemical anatomy of the afferent vagal system. Auton Neurosci 85:1-17. 
Berthoud HR, Powley TL (1992) Vagal afferent innervation of the rat fundic stomach: morphological characterization of the gastric tension receptor. J Comp Neurol 319:261276.

Bethea JR, Castro M, Keane RW, Lee TT, Dietrich WD, Yezierski RP (1998) Traumatic spinal cord injury induces nuclear factor-kappaB activation. J Neurosci 18:3251-3260.

Bielefeldt K, Christianson JA, Davis BM (2005) Basic and clinical aspects of visceral sensation: transmission in the CNS. Neurogastroenterol Motil 17:488-499.

Bielefeldt K, Lamb K, Gebhart GF (2006a) Convergence of sensory pathways in the development of somatic and visceral hypersensitivity. Am J Physiol Gastrointest Liver Physiol 291:G658-665.

Bielefeldt K, Zhong F, Koerber HR, Davis BM (2006b) Phenotypic characterization of gastric sensory neurons in mice. Am J Physiol Gastrointest Liver Physiol 291:G987-997.

Birder L, Andersson KE (2013) Urothelial signaling. Physiol Rev 93:653-680.

Birder LA (2010) Urothelial signaling. Auton Neurosci 153:33-40.

Birder LA (2011) Urothelial signaling. Handb Exp Pharmacol 207-231.

Birder LA, Ruggieri M, Takeda M, van Koeveringe G, Veltkamp S, Korstanje C, Parsons B, Fry CH (2012) How does the urothelium affect bladder function in health and disease? ICI-RS 2011. Neurourol Urodyn 31:293-299.

Blok BF, de Weerd H, Holstege G (1997a) The pontine micturition center projects to sacral cord GABA immunoreactive neurons in the cat. Neurosci Lett 233:109-112.

Blok BF, Sturms LM, Holstege G (1997b) A PET study on cortical and subcortical control of pelvic floor musculature in women. J Comp Neurol 389:535-544.

Blok BF, Sturms LM, Holstege G (1998) Brain activation during micturition in women. Brain 121 ( Pt 11):2033-2042.

Bobrow MN, Harris TD, Shaughnessy KJ, Litt GJ (1989) Catalyzed reporter deposition, a novel method of signal amplification. Application to immunoassays. J Immunol Methods 125:279-285.

Bobrow MN, Litt GJ, Shaughnessy KJ, Mayer PC, Conlon J (1992) The use of catalyzed reporter deposition as a means of signal amplification in a variety of formats. J Immunol Methods 150:145-149.

Bobrow MN, Shaughnessy KJ, Litt GJ (1991) Catalyzed reporter deposition, a novel method of signal amplification. II. Application to membrane immunoassays. J Immunol Methods 137:103-112.

Bogen O, Dina OA, Gear RW, Levine JD (2009) Dependence of monocyte chemoattractant protein 1 induced hyperalgesia on the isolectin B4-binding protein versican. Neuroscience 159:780-786.

Bogen O, Dreger M, Gillen C, Schroder W, Hucho F (2005) Identification of versican as an isolectin B4-binding glycoprotein from mammalian spinal cord tissue. The FEBS journal 272:1090-1102.

Borra E, Belmalih A, Gerbella M, Rozzi S, Luppino G (2010) Projections of the hand field of the macaque ventral premotor area F5 to the brainstem and spinal cord. J Comp Neurol 518:2570-2591.

Bouvier M, Grimaud JC, Abysique A (1990) Effects of stimulation of vesical afferents on colonic motility in cats. Gastroenterology 98:1148-1154. 
Bradbury EJ, Burnstock G, McMahon SB (1998) The expression of P2X3 purinoreceptors in sensory neurons: effects of axotomy and glial-derived neurotrophic factor. Mol Cell Neurosci 12:256-268.

Broussard DL, Altschuler SM (2000) Brainstem viscerotopic organization of afferents and efferents involved in the control of swallowing. Am J Med 108 Suppl 4a:79S-86S.

Browning KN, Mendelowitz D (2003) Musings on the wanderer: what's new in our understanding of vago-vagal reflexes?: II. Integration of afferent signaling from the viscera by the nodose ganglia. Am J Physiol Gastrointest Liver Physiol 284:G8-14.

Brumovsky PR, Gebhart GF (2010) Visceral organ cross-sensitization - an integrated perspective. Auton Neurosci 153:106-115.

Bueno L, Fioramonti J (2002) Visceral perception: inflammatory and non-inflammatory mediators. Gut 51 Suppl 1:i19-23.

Burden HW, Leonard M, Smith CP, Lawrence IE, Jr. (1983) The sensory innervation of the ovary: a horseradish peroxidase study in the rat. Anat Rec 207:623-627.

Burnstock G (1996) A unifying purinergic hypothesis for the initiation of pain. Lancet 347:16041605.

Burnstock G (2007) Physiology and pathophysiology of purinergic neurotransmission. Physiol Rev 87:659-797.

Burnstock G (2014) Purinergic signalling in the urinary tract in health and disease. Purinergic Signal 10:103-155.

Campbell SJ, Perry VH, Pitossi FJ, Butchart AG, Chertoff M, Waters S, Dempster R, Anthony DC (2005) Central nervous system injury triggers hepatic CC and CXC chemokine expression that is associated with leukocyte mobilization and recruitment to both the central nervous system and the liver. Am J Pathol 166:1487-1497.

Carobi C (1987) Capsaicin-sensitive vagal afferent neurons innervating the rat pancreas. Neurosci Lett 77:5-9.

Cartwright R, Afshan I, Derpapas A, Vijaya G, Khullar V (2011) Novel biomarkers for overactive bladder. Nature reviews Urology 8:139-145.

Castoro MA, Yoo PB, Hincapie JG, Hamann JJ, Ruble SB, Wolf PD, Grill WM (2011) Excitation properties of the right cervical vagus nerve in adult dogs. Exp Neurol 227:62-68.

Cechetto DF (1995) Supraspinal mechanisms of visceral representation. In: Visceral Pain, Progress in Pain Research and Management, vol. 5 (Gebhart, G. F., ed), pp 261-290 Seattle, Wa.: IASP.

Cervero F (1994) Sensory innervation of the viscera: peripheral basis of visceral pain. Physiol Rev 74:95-138.

Chandler MJ, Zhang J, Foreman RD (1996) Vagal, sympathetic and somatic sensory inputs to upper cervical (C1-C3) spinothalamic tract neurons in monkeys. J Neurophysiol 76:25552567.

Chen CC, Akopian AN, Sivilotti L, Colquhoun D, Burnstock G, Wood JN (1995) A P2X purinoceptor expressed by a subset of sensory neurons. Nature 377:428-431.

Chen XQ, Wang B, Wu C, Pan J, Yuan B, Su YY, Jiang XY, Zhang X, Bao L (2012) Endosomemediated retrograde axonal transport of $\mathrm{P} 2 \mathrm{X} 3$ receptor signals in primary sensory neurons. Cell Res 22:677-696.

Cheng Z, Powley TL, Schwaber JS, Doyle FJ, 3rd (1997) Vagal afferent innervation of the atria of the rat heart reconstructed with confocal microscopy. J Comp Neurol 381:1-17. 
Christian EP, Togo JA, Naper KE, Koschorke G, Taylor GA, Weinreich D (1993) A retrograde labeling technique for the functional study of airway-specific visceral afferent neurons. J Neurosci Methods 47:147-160.

Christianson JA, Liang R, Ustinova EE, Davis BM, Fraser MO, Pezzone MA (2007) Convergence of bladder and colon sensory innervation occurs at the primary afferent level. Pain 128:235-243.

Christianson JA, Traub RJ, Davis BM (2006) Differences in spinal distribution and neurochemical phenotype of colonic afferents in mouse and rat. J Comp Neurol 494:246-259.

Cockayne DA, Hamilton SG, Zhu QM, Dunn PM, Zhong Y, Novakovic S, Malmberg AB, Cain G, Berson A, Kassotakis L, Hedley L, Lachnit WG, Burnstock G, McMahon SB, Ford AP (2000) Urinary bladder hyporeflexia and reduced pain-related behaviour in P2X3-deficient mice. Nature 407:1011-1015.

Coggeshall RE, Lekan HA (1996) Methods for determining numbers of cells and synapses: a case for more uniform standards of review. J Comp Neurol 364:6-15.

Coleridge JC, Coleridge HM (1984) Afferent vagal C fibre innervation of the lungs and airways and its functional significance. Reviews of physiology, biochemistry and pharmacology 99:1-110.

Collins JJ, Lin CE, Berthoud HR, Papka RE (1999) Vagal afferents from the uterus and cervix provide direct connections to the brainstem. Cell Tissue Res 295:43-54.

Cook SP, McCleskey EW (2002) Cell damage excites nociceptors through release of cytosolic ATP. Pain 95:41-47.

Cook SP, Vulchanova L, Hargreaves KM, Elde R, McCleskey EW (1997) Distinct ATP receptors on pain-sensing and stretch-sensing neurons. Nature 387:505-508.

Cottle MK (1964) DEGENERATION STUDIES OF PRIMARY AFFERENTS OF IXTH AND XTH CRANIAL NERVES IN THE CAT. J Comp Neurol 122:329-345.

Cristancho P, Cristancho MA, Baltuch GH, Thase ME, O'Reardon JP (2011) Effectiveness and safety of vagus nerve stimulation for severe treatment-resistant major depression in clinical practice after FDA approval: outcomes at 1 year. J Clin Psychiatry.

D'Agostino G, Condino AM, Calvi V, Boschi F, Gioglio L, Barbieri A (2012) Purinergic P2X3 heteroreceptors enhance parasympathetic motor drive in isolated porcine detrusor, a reliable model for development of P2X selective blockers for detrusor hyperactivity. Pharmacological research : the official journal of the Italian Pharmacological Society 65:129-136.

D'Arco M, Giniatullin R, Simonetti M, Fabbro A, Nair A, Nistri A, Fabbretti E (2007) Neutralization of nerve growth factor induces plasticity of ATP-sensitive P2X3 receptors of nociceptive trigeminal ganglion neurons. J Neurosci 27:8190-8201.

Dampney RA (1994) Functional organization of central pathways regulating the cardiovascular system. Physiol Rev 74:323-364.

Dang K, Bielfeldt K, Lamb K, Gebhart GF (2005) Gastric ulcers evoke hyperexcitability and enhance P2X receptor function in rat gastric sensory neurons. J Neurophysiol 93:31123119.

Dang K, Lamb K, Cohen M, Bielefeldt K, Gebhart GF (2008) Cyclophosphamide-induced bladder inflammation sensitizes and enhances $\mathrm{P} 2 \mathrm{X}$ receptor function in rat bladder sensory neurons. J Neurophysiol 99:49-59. 
Danzer M, Jocic M, Samberger C, Painsipp E, Bock E, Pabst MA, Crailsheim K, Schicho R, Lippe IT, Holzer $P$ (2004) Stomach-brain communication by vagal afferents in response to luminal acid backdiffusion, gastrin, and gastric acid secretion. Am J Physiol Gastrointest Liver Physiol 286:G403-411.

de Groat WC (1987a) Neuropeptides in pelvic afferent pathways. Experientia 43:801-813. de Groat WC (1995) Mechanisms underlying the recovery of lower urinary tract function following spinal cord injury. Paraplegia 33:493-505.

de Groat WC, Kawatani M, Hisamitsu T, Cheng CL, Ma CP, Thor K, Steers W, Roppolo JR (1990) Mechanisms underlying the recovery of urinary bladder function following spinal cord injury. J Auton Nerv Syst 30 Suppl:S71-77.

De Groat WC, Kawatani, M., Houston, M.B., Rutigliiano, M., Erdman, S., (1987b) Identification of neuropeptides in afferent pathways to the pelvic viscera of the cat. In: Organization of the autonomic nervous system: central and peripheral mechanisms (Ciriello, J., Calaresu, F., Renaud, L., Polosa, C., ed), pp 81-90 New York: A.R. Liss.

de Groat WC, Kruse MN, Vizzard MA, Cheng CL, Araki I, Yoshimura N (1997) Modification of urinary bladder function after spinal cord injury. Adv Neurol 72:347-364.

de Groat WC, Yoshimura N (2001) Pharmacology of the lower urinary tract. Annu Rev Pharmacol Toxicol 41:691-721.

de Groat WC, Yoshimura N (2006) Mechanisms underlying the recovery of lower urinary tract function following spinal cord injury. Prog Brain Res 152:59-84.

de Groat WC, Yoshimura N (2010) Changes in afferent activity after spinal cord injury. Neurourol Urodyn 29:63-76.

De Looze D, Van Laere M, De Muynck M, Beke R, Elewaut A (1998) Constipation and other chronic gastrointestinal problems in spinal cord injury patients. Spinal Cord 36:63-66.

De Wachter S, de Jong A, Van Dyck J, Wyndaele JJ (2007) Interaction of filling related sensation between anorectum and lower urinary tract and its impact on the sequence of their evacuation. A study in healthy volunteers. Neurourol Urodyn 26:481-485.

De Wachter S, Wyndaele JJ (2003) Impact of rectal distention on the results of evaluations of lower urinary tract sensation. J Urol 169:1392-1394.

deCampo DM, Fudge JL (2013) Amygdala projections to the lateral bed nucleus of the stria terminalis in the macaque: comparison with ventral striatal afferents. J Comp Neurol 521:3191-3216.

Deng JB, Yu DM, Wu P, Li MS (2007) The tracing study of developing entorhino-hippocampal pathway. Int J Dev Neurosci 25:251-258.

Denny-Brown D, Robertson, E.G. (1933) On the physiology of micturition. Brain 56:149-190

Ditunno PL, Patrick M, Stineman M, Ditunno JF (2008) Who wants to walk? Preferences for recovery after SCI: a longitudinal and cross-sectional study. Spinal Cord 46:500-506.

Djouhri L, Bleazard L, Lawson SN (1998) Association of somatic action potential shape with sensory receptive properties in guinea-pig dorsal root ganglion neurones. J Physiol 513 ( Pt 3):857-872.

Dmitrieva N, Berkley KJ (2002) Contrasting effects of WIN 55212-2 on motility of the rat bladder and uterus. J Neurosci 22:7147-7153.

Dmitrieva N, Johnson OL, Berkley KJ (2001) Bladder inflammation and hypogastric neurectomy influence uterine motility in the rat. Neurosci Lett 313:49-52. 
Domotor A, Peidl Z, Vincze A, Hunyady B, Szolcsanyi J, Kereskay L, Szekeres G, Mozsik G (2005) Immunohistochemical distribution of vanilloid receptor, calcitonin-gene related peptide and substance $P$ in gastrointestinal mucosa of patients with different gastrointestinal disorders. Inflammopharmacology 13:161-177.

Dutsch M, Eichhorn U, Worl J, Wank M, Berthoud HR, Neuhuber WL (1998) Vagal and spinal afferent innervation of the rat esophagus: a combined retrograde tracing and immunocytochemical study with special emphasis on calcium-binding proteins. J Comp Neurol 398:289-307.

Elbadawi A, Schenk EA (1974) A new theory of the innervation of bladder musculature. 4. Innervation of the vesicourethral junction and external urethral sphincter. J Urol 111:613-615.

Elneil S, Skepper JN, Kidd EJ, Williamson JG, Ferguson DR (2001) Distribution of P2X(1) and $P 2 X(3)$ receptors in the rat and human urinary bladder. Pharmacology 63:120-128.

Eriksson J, Bongenhielm U, Kidd E, Matthews B, Fried K (1998) Distribution of P2X3 receptors in the rat trigeminal ganglion after inferior alveolar nerve injury. Neurosci Lett 254:37-40.

Ersoz M, Akyuz M (2004) Bladder-filling sensation in patients with spinal cord injury and the potential for sensation-dependent bladder emptying. Spinal Cord 42:110-116.

Evans RJ, Moldwin RM, Cossons N, Darekar A, Mills IW, Scholfield D (2011) Proof of concept trial of tanezumab for the treatment of symptoms associated with interstitial cystitis. J Urol 185:1716-1721.

Fabbretti E, D'Arco M, Fabbro A, Simonetti M, Nistri A, Giniatullin R (2006) Delayed upregulation of ATP P2X3 receptors of trigeminal sensory neurons by calcitonin gene-related peptide. J Neurosci 26:6163-6171.

Fang M, Wang J, Huang JY, Ling SC, Rudd JA, Hu ZY, Yew DT, Han S (2011) The neuroprotective effects of Reg-2 following spinal cord transection injury. Anatomical record (Hoboken, NJ : 2007) 294:24-45.

Fasanella KE, Christianson JA, Chanthaphavong RS, Davis BM (2008) Distribution and neurochemical identification of pancreatic afferents in the mouse. J Comp Neurol 509:42-52.

Fernandez O (2002) Mechanisms and current treatments of urogenital dysfunction in multiple sclerosis. Journal of neurology 249:1-8.

Fleming JC, Norenberg MD, Ramsay DA, Dekaban GA, Marcillo AE, Saenz AD, Pasquale-Styles M, Dietrich WD, Weaver LC (2006) The cellular inflammatory response in human spinal cords after injury. Brain 129:3249-3269.

Floyd K, McMahon SB, Morrison JF (1978) Inhibition of the micturition reflex by stimulation of pelvic nerve afferents from the colon [proceedings]. J Physiol 284:39P-40P.

Floyd K, McMahon SB, Morrison JF (1979) Inhibitory interactions between the colonic and vesical branches of the pelvic nerve in the cat [proceedings]. J Physiol 290:50P-51P.

Floyd K, McMahon SB, Morrison JF (1982) Inhibitory interactions between colonic and vesical afferents in the micturition reflex of the cat. J Physiol 322:45-52.

Ford AP, Gever JR, Nunn PA, Zhong Y, Cefalu JS, Dillon MP, Cockayne DA (2006) Purinoceptors as therapeutic targets for lower urinary tract dysfunction. Br J Pharmacol 147 Suppl 2:S132-143.

Fowler CJ, Griffiths D, de Groat WC (2008) The neural control of micturition. Nat Rev Neurosci 9:453-466. 
Frankenhaüser I (1866) Nerven der weiblichen Geschlechtorgane. Jena Ztschr.

Fry CH, Sahai A, Vahabi B, Kanai AJ, Birder LA (2014) What is the role for biomarkers for lower urinary tract disorders? ICI-RS 2013. Neurourol Urodyn 33:602-605.

Fu QG, Chandler MJ, McNeill DL, Foreman RD (1992) Vagal afferent fibers excite upper cervical neurons and inhibit activity of lumbar spinal cord neurons in the rat. Pain 51:91-100.

Furuta A, Jankowski RJ, Honda M, Pruchnic R, Yoshimura N, Chancellor MB (2007) State of the art of where we are at using stem cells for stress urinary incontinence. Neurourol Urodyn 26:966-971.

Gallego R, Eyzaguirre C (1978) Membrane and action potential characteristics of A and C nodose ganglion cells studied in whole ganglia and in tissue slices. J Neurophysiol 41:1217-1232.

Gattone VH, 2nd, Marfurt CF, Dallie S (1986) Extrinsic innervation of the rat kidney: a retrograde tracing study. Am J Physiol 250:F189-196.

Gebhart GF, Bielefeldt K, Ozaki N (2002) Gastric hyperalgesia and changes in voltage gated sodium channel function in the rat. Gut 51 Suppl 1:i15-18.

Giamberardino MA, De Laurentis S, Affaitati G, Lerza R, Lapenna D, Vecchiet L (2001) Modulation of pain and hyperalgesia from the urinary tract by algogenic conditions of the reproductive organs in women. Neurosci Lett 304:61-64.

Giniatullin R, Nistri A, Fabbretti E (2008) Molecular mechanisms of sensitization of paintransducing P2X3 receptors by the migraine mediators CGRP and NGF. Mol Neurobiol 37:83-90.

Gittins R, Harrison PJ (2004) Neuronal density, size and shape in the human anterior cingulate cortex: a comparison of Nissl and NeuN staining. Brain Res Bull 63:155-160.

Glick ME, Meshkinpour H, Haldeman S, Hoehler F, Downey N, Bradley WE (1984) Colonic dysfunction in patients with thoracic spinal cord injury. Gastroenterology 86:287-294.

Godement P, Vanselow J, Thanos S, Bonhoeffer F (1987) A study in developing visual systems with a new method of staining neurones and their processes in fixed tissue. Development 101:697-713.

Gold MS, Zhang L, Wrigley DL, Traub RJ (2002) Prostaglandin E(2) modulates TTX-R I(Na) in rat colonic sensory neurons. J Neurophysiol 88:1512-1522.

Gondim FA, Alencar HM, Rodrigues CL, da Graca JR, dos Santos AA, Rola FH (1999) Complete cervical or thoracic spinal cord transections delay gastric emptying and gastrointestinal transit of liquid in awake rats. Spinal Cord 37:793-799.

Gondim FA, da-Graca JR, de-Oliveira GR, Rego MC, Gondim RB, Rola FH (1998) Decreased gastric emptying and gastrointestinal and intestinal transits of liquid after complete spinal cord transection in awake rats. Braz J Med Biol Res 31:1605-1610.

Gondim FA, Rodrigues CL, da Graca JR, Camurca FD, de Alencar HM, dos Santos AA, Rola FH (2001) Neural mechanisms involved in the delay of gastric emptying and gastrointestinal transit of liquid after thoracic spinal cord transection in awake rats. Auton Neurosci 87:52-58.

Gore RM, Mintzer RA, Calenoff L (1981) Gastrointestinal complications of spinal cord injury. Spine (Phila Pa 1976) 6:538-544.

Grabauskas G, Song I, Zhou S, Owyang C (2010) Electrophysiological identification of glucosesensing neurons in rat nodose ganglia. J Physiol 588:617-632.

Green T, Dockray GJ (1987) Calcitonin gene-related peptide and substance $P$ in afferents to the upper gastrointestinal tract in the rat. Neurosci Lett 76:151-156. 
Gris D, Hamilton EF, Weaver LC (2008) The systemic inflammatory response after spinal cord injury damages lungs and kidneys. Exp Neurol 211:259-270.

Groves DA, Brown VJ (2005) Vagal nerve stimulation: a review of its applications and potential mechanisms that mediate its clinical effects. Neurosci Biobehav Rev 29:493-500.

Grundy D (2002) Neuroanatomy of visceral nociception: vagal and splanchnic afferent. Gut 51 Suppl 1:i2-5.

Habler HJ, Janig W, Koltzenburg M (1990) Activation of unmyelinated afferent fibres by mechanical stimuli and inflammation of the urinary bladder in the cat. J Physiol 425:545562.

Hai T, Wolfgang CD, Marsee DK, Allen AE, Sivaprasad U (1999) ATF3 and stress responses. Gene expression 7:321-335.

Hammell KR (2010) Spinal cord injury rehabilitation research: patient priorities, current deficiencies and potential directions. Disability and rehabilitation 32:1209-1218.

Hansen RB, Biering-Sorensen F, Kristensen JK (2010) Urinary incontinence in spinal cord injured individuals 10-45 years after injury. Spinal Cord 48:27-33.

Hartman MG, Lu D, Kim ML, Kociba GJ, Shukri T, Buteau J, Wang X, Frankel WL, Guttridge D, Prentki M, Grey ST, Ron D, Hai T (2004) Role for activating transcription factor 3 in stress-induced beta-cell apoptosis. Molecular and cellular biology 24:5721-5732.

Hausmann ON (2003) Post-traumatic inflammation following spinal cord injury. Spinal Cord 41:369-378.

Hayakawa T, Kuwahara-Otani S, Maeda S, Tanaka K, Seki M (2011) Projections of calcitonin gene-related peptide immunoreactive neurons in the vagal ganglia of the rat. J Chem Neuroanat 41:55-62.

Hayakawa T, Kuwahara S, Maeda S, Tanaka K, Seki M (2010) Calcitonin gene-related peptide immunoreactive neurons innervating the soft palate, the root of tongue, and the pharynx in the superior glossopharyngeal ganglion of the rat. J Chem Neuroanat 39:221227.

Hays WL (1981) Statistics. New York: Holt, Rinehart and Winston.

Helke CJ (2005) Vagal afferent neurons: Neurotrophic factors and epigenetic influences. Boca Raton, FL: CRC Press Taylor and Francis Group.

Helke CJ, Adryan KM, Fedorowicz J, Zhuo H, Park JS, Curtis R, Radley HE, Distefano PS (1998) Axonal transport of neurotrophins by visceral afferent and efferent neurons of the vagus nerve of the rat. J Comp Neurol 393:102-117.

Helke CJ, Hill KM (1988) Immunohistochemical study of neuropeptides in vagal and glossopharyngeal afferent neurons in the rat. Neuroscience 26:539-551.

Helke CJ, Niederer AJ (1990) Studies on the coexistence of substance P with other putative transmitters in the nodose and petrosal ganglia. Synapse 5:144-151.

Helke CJ, O'Donohue TL, Jacobowitz DM (1980) Substance P as a baro- and chemoreceptor afferent neurotransmitter: immunocytochemical and neurochemical evidence in the rat. Peptides 1:1-9.

Helke CJ, Rabchevsky A (1991) Axotomy alters putative neurotransmitters in visceral sensory neurons of the nodose and petrosal ganglia. Brain Res 551:44-51.

Herbert H, Saper CB (1992) Organization of medullary adrenergic and noradrenergic projections to the periaqueductal gray matter in the rat. J Comp Neurol 315:34-52. 
Herculano-Houzel S, Lent R (2005) Isotropic fractionator: a simple, rapid method for the quantification of total cell and neuron numbers in the brain. J Neurosci 25:2518-2521.

Herrera JJ, Haywood-Watson RJ, 2nd, Grill RJ (2010) Acute and chronic deficits in the urinary bladder after spinal contusion injury in the adult rat. J Neurotrauma 27:423-431.

Herrity AN, Rau KK, Petruska JC, Stirling DP, Hubscher CH (2014) Identification of bladder and colon afferents in the nodose ganglia of male rats. J Comp Neurol 522:3667-3682.

Heyningen SV (1974) Choleratoxin: interaction of subunits with ganglioside GM1. Science 183:656-657.

Hill CE, Harrison BJ, Rau KK, Hougland MT, Bunge MB, Mendell LM, Petruska JC (2010) Skin incision induces expression of axonal regeneration-related genes in adult rat spinal sensory neurons. J Pain 11:1066-1073.

Holmes GM (2012) Upper gastrointestinal dysmotility after spinal cord injury: is diminished vagal sensory processing one culprit? Frontiers in physiology 3:277.

Holstege $G$ (2005) Micturition and the soul. J Comp Neurol 493:15-20.

Holstege G, Griffiths D, de Wall H, Dalm E (1986) Anatomical and physiological observations on supraspinal control of bladder and urethral sphincter muscles in the cat. J Comp Neurol 250:449-461.

Holstege G, Kuypers HG (1982) The anatomy of brain stem pathways to the spinal cord in cat. A labeled amino acid tracing study. Prog Brain Res 57:145-175.

Holstege G, Kuypers HG, Boer RC (1979) Anatomical evidence for direct brain stem projections to the somatic motoneuronal cell groups and autonomic preganglionic cell groups in cat spinal cord. Brain Res 171:329-333.

Holzer P (2003) Afferent signalling of gastric acid challenge. J Physiol Pharmacol 54 Suppl 4:4353.

Holzer P, Danzer M, Schicho R, Samberger C, Painsipp E, Lippe IT (2004) Vagal afferent input from the acid-challenged rat stomach to the brainstem: enhancement by interleukin1beta. Neuroscience 129:439-445.

Hong CC, Henderson FC (1989) Urinary tract infections associated with spinal cord injury in rats. J Med Sci 9:317-325.

Honig MG, Hume RI (1986) Fluorescent carbocyanine dyes allow living neurons of identified origin to be studied in long-term cultures. The Journal of cell biology 103:171-187.

Honig MG, Hume RI (1989) Dil and diO: versatile fluorescent dyes for neuronal labelling and pathway tracing. Trends Neurosci 12:333-335, 340-331.

Hoover DB, Shepherd AV, Southerland EM, Armour JA, Ardell JL (2008) Neurochemical diversity of afferent neurons that transduce sensory signals from dog ventricular myocardium. Auton Neurosci 141:38-45.

Hoyle CH (1994) Non-adrenergic, non-cholinergic control of the urinary bladder. World journal of urology 12:233-244.

Hubscher CH, Berkley KJ (1994) Responses of neurons in caudal solitary nucleus of female rats to stimulation of vagina, cervix, uterine horn and colon. Brain Res 664:1-8.

Hubscher CH, Berkley KJ (1995) Spinal and vagal influences on the responses of rat solitary nucleus neurons to stimulation of uterus, cervix and vagina. Brain Res 702:251-254.

Hubscher CH, Johnson RD (2000) Effects of acute and chronic midthoracic spinal cord injury on neural circuits for male sexual function. II. Descending pathways. J Neurophysiol 83:2508-2518. 
Hubscher CH, Kaddumi EG, Johnson RD (2004) Brain stem convergence of pelvic viscerosomatic inputs via spinal and vagal afferents. Neuroreport 15:1299-1302.

Hubscher CH, Petruska JC, Rau KK, Johnson RD (2001) Co-expression of P2X receptor subunits on rat nodose neurons that bind the isolectin GS-I-B4. Neuroreport 12:2995-2997.

Huynh HK, Willemsen AT, Lovick TA, Holstege G (2013) Pontine control of ejaculation and female orgasm. The journal of sexual medicine 10:3038-3048.

Ichikawa $\mathrm{H}$, Helke CJ (1999) The coexistence of TrkA with putative transmitter agents and calcium-binding proteins in the vagal and glossopharyngeal sensory neurons of the adult rat. Brain Res 846:268-273.

Ikeda SR, Schofield GG, Weight FF (1986) Na+ and Ca2+ currents of acutely isolated adult rat nodose ganglion cells. J Neurophysiol 55:527-539.

Inoue T, Gabella G (1992) The interface between epithelium and lamina propria in the rat urinary bladder. Archives of histology and cytology 55 Suppl:157-163.

Jaffe RA, Sampson SR (1976) Analysis of passive and active electrophysiologic properties of neurons in mammalian nodose ganglia maintained in vitro. J Neurophysiol 39:802-815.

Jancso G, Maggi CA (1987) Distribution of capsaicin-sensitive urinary bladder afferents in the rat spinal cord. Brain Res 418:371-376.

Janig W (1996) Neurobiology of visceral afferent neurons: neuroanatomy, functions, organ regulations and sensations. Biological psychology 42:29-51.

Jordan D (2001) Central nervous pathways and control of the airways. Respiration physiology 125:67-81.

Kaddumi EG, Hubscher CH (2006) Convergence of multiple pelvic organ inputs in the rat rostral medulla. J Physiol 572:393-405.

Kaddumi EG, Hubscher CH (2007) Urinary bladder irritation alters efficacy of vagal stimulation on rostral medullary neurons in chronic T8 spinalized rats. J Neurotrauma 24:1219-1228.

Kaddumi EG, Qnais EY, Allouh MZ (2012) Effect of esophagus distention on urinary bladder function in rats. Neurourol Urodyn 31:174-177.

Kalia M, Mesulam MM (1980) Brain stem projections of sensory and motor components of the vagus complex in the cat: II. Laryngeal, tracheobronchial, pulmonary, cardiac, and gastrointestinal branches. J Comp Neurol 193:467-508.

Kanai A, Andersson KE (2010) Bladder afferent signaling: recent findings. J Urol 183:1288-1295.

Kaneko T, Saeki K, Lee T, Mizuno N (1996) Improved retrograde axonal transport and subsequent visualization of tetramethylrhodamine (TMR) -dextran amine by means of an acidic injection vehicle and antibodies against TMR. J Neurosci Methods 65:157-165.

Kao CH, Ho YJ, Changlai SP, Ding HJ (1999) Gastric emptying in spinal cord injury patients. Dig Dis Sci 44:1512-1515.

Kashyap M, Kawamorita N, Tyagi V, Sugino Y, Chancellor M, Yoshimura N, Tyagi P (2013) Downregulation of nerve growth factor expression in the bladder by antisense oligonucleotides as new treatment for overactive bladder. J Urol 190:757-764.

Kawauchi J, Zhang C, Nobori K, Hashimoto Y, Adachi MT, Noda A, Sunamori M, Kitajima S (2002) Transcriptional repressor activating transcription factor 3 protects human umbilical vein endothelial cells from tumor necrosis factor-alpha-induced apoptosis through downregulation of p53 transcription. J Biol Chem 277:39025-39034. 
Keast JR, De Groat WC (1992) Segmental distribution and peptide content of primary afferent neurons innervating the urogenital organs and colon of male rats. J Comp Neurol 319:615-623.

Kestler C, Neuhuber WL, Raab M (2009) Distribution of P2X(3) receptor immunoreactivity in myenteric ganglia of the mouse esophagus. Histochem Cell Biol 131:13-27.

Khakh BS, North RA (2006) P2X receptors as cell-surface ATP sensors in health and disease. Nature 442:527-532.

King BF, Knowles ID, Burnstock G, Ramage AG (2004) Investigation of the effects of P2 purinoceptor ligands on the micturition reflex in female urethane-anaesthetized rats. $\mathrm{Br}$ J Pharmacol 142:519-530.

Kirchberger I, Biering-Sorensen F, Charlifue S, Baumberger M, Campbell R, Kovindha A, Ring $H$, Sinnott A, Scheuringer M, Stucki G (2010) Identification of the most common problems in functioning of individuals with spinal cord injury using the International Classification of Functioning, Disability and Health. Spinal Cord 48:221-229.

Klop EM, Mouton LJ, Kuipers R, Holstege G (2005) Neurons in the lateral sacral cord of the cat project to periaqueductal grey, but not to thalamus. Eur J Neurosci 21:2159-2166.

Klusman I, Schwab ME (1997) Effects of pro-inflammatory cytokines in experimental spinal cord injury. Brain Res 762:173-184.

Knowles CH, Aziz Q (2009) Basic and clinical aspects of gastrointestinal pain. Pain 141:191-209.

Knowlton GC, Larrabee MG (1946) A unitary analysis of pulmonary volume receptors. Am J Physiol 147:100-114.

Kobbert C, Apps R, Bechmann I, Lanciego JL, Mey J, Thanos S (2000) Current concepts in neuroanatomical tracing. Prog Neurobiol 62:327-351.

Koerber HR, Mirnics K, Kavookjian AM, Light AR (1999) Ultrastructural analysis of ectopic synaptic boutons arising from peripherally regenerated primary afferent fibers. J Neurophysiol 81:1636-1644.

Komisaruk BR, Gerdes CA, Whipple B (1997) 'Complete' spinal cord injury does not block perceptual responses to genital self-stimulation in women. Arch Neurol 54:1513-1520.

Komisaruk BR, Whipple B, Crawford A, Liu WC, Kalnin A, Mosier K (2004) Brain activation during vaginocervical self-stimulation and orgasm in women with complete spinal cord injury: fMRI evidence of mediation by the vagus nerves. Brain Res 1024:77-88.

Kowski AB, Geisler S, Krauss M, Veh RW (2008) Differential projections from subfields in the lateral preoptic area to the lateral habenular complex of the rat. J Comp Neurol 507:1465-1478.

Krhut J HP, Tintera J, Zachoval R, Bilkova K, Zvara P, Blok B (2014) Brain activity associated with urinary bladder filling in patients with a complete spinal cord injury (SCI) - a FMRI study. In: ICS Rio de Janeiro.

Kruse MN, Bray LA, de Groat WC (1995) Influence of spinal cord injury on the morphology of bladder afferent and efferent neurons. J Auton Nerv Syst 54:215-224.

Kruse MN, de Groat WC (1993) Changes in lower urinary tract function following spinal cord injury. Restorative neurology and neuroscience 5:79-80.

Kubin L, Alheid GF, Zuperku EJ, McCrimmon DR (2006) Central pathways of pulmonary and lower airway vagal afferents. Journal of applied physiology (Bethesda, Md : 1985) 101:618627. 
Kuipers R, Mouton LJ, Holstege G (2006) Afferent projections to the pontine micturition center in the cat. J Comp Neurol 494:36-53.

Kummer W, Fischer A, Kurkowski R, Heym C (1992) The sensory and sympathetic innervation of guinea-pig lung and trachea as studied by retrograde neuronal tracing and doublelabelling immunohistochemistry. Neuroscience 49:715-737.

Kuo HC, Liu HT, Chancellor MB (2010) Urinary nerve growth factor is a better biomarker than detrusor wall thickness for the assessment of overactive bladder with incontinence. Neurourol Urodyn 29:482-487.

Labar D, Murphy J, Tecoma E (1999) Vagus nerve stimulation for medication-resistant generalized epilepsy. E04 VNS Study Group. Neurology 52:1510-1512.

Lamb K, Bielefeldt K (2003) Rapid effects of neurotrophic factors on calcium homeostasis in rat visceral afferent neurons. Neurosci Lett 336:9-12.

Lamb K, Kang YM, Gebhart GF, Bielefeldt K (2003) Gastric inflammation triggers hypersensitivity to acid in awake rats. Gastroenterology 125:1410-1418.

Lamb K, Zhong F, Gebhart GF, Bielefeldt K (2006) Experimental colitis in mice and sensitization of converging visceral and somatic afferent pathways. Am J Physiol Gastrointest Liver Physiol 290:G451-457.

Langford LA, Coggeshall RE (1981) Branching of sensory axons in the peripheral nerve of the rat. J Comp Neurol 203:745-750.

Lawson SN, Crepps B, Perl ER (2002) Calcitonin gene-related peptide immunoreactivity and afferent receptive properties of dorsal root ganglion neurones in guinea-pigs. J Physiol 540:989-1002.

Lei Q, Malykhina AP (2012) Colonic inflammation up-regulates voltage-gated sodium channels in bladder sensory neurons via activation of peripheral transient potential vanilloid 1 receptors. Neurogastroenterol Motil 24:575-585, e257.

Lei Q, Pan XQ, Villamor AN, Asfaw TS, Chang S, Zderic SA, Malykhina AP (2013) Lack of transient receptor potential vanilloid 1 channel modulates the development of neurogenic bladder dysfunction induced by cross-sensitization in afferent pathways. Journal of neuroinflammation 10:3.

Lewis C, Neidhart S, Holy C, North RA, Buell G, Surprenant A (1995) Coexpression of P2X2 and P2X3 receptor subunits can account for ATP-gated currents in sensory neurons. Nature 377:432-435.

Li BY, Schild JH (2007) Electrophysiological and pharmacological validation of vagal afferent fiber type of neurons enzymatically isolated from rat nodose ganglia. J Neurosci Methods 164:75-85.

Li H, Nomura S, Mizuno N (1997) Binding of the isolectin I-B4 from Griffonia simplicifolia to the general visceral afferents in the vagus nerve: a light- and electron-microscope study in the rat. Neurosci Lett 222:53-56.

Li L, Zhou XF (2001) Pericellular Griffonia simplicifolia I isolectin B4-binding ring structures in the dorsal root ganglia following peripheral nerve injury in rats. J Comp Neurol 439:259-274.

Li M, Zheng C, Sato T, Kawada T, Sugimachi M, Sunagawa K (2004) Vagal nerve stimulation markedly improves long-term survival after chronic heart failure in rats. Circulation 109:120-124. 
Li WP, Xian C, Rush RA, Zhou XF (1999) Upregulation of brain-derived neurotrophic factor and neuropeptide $Y$ in the dorsal ascending sensory pathway following sciatic nerve injury in rat. Neurosci Lett 260:49-52.

Liang R, Ustinova EE, Patnam R, Fraser MO, Gutkin DW, Pezzone MA (2007) Enhanced expression of mast cell growth factor and mast cell activation in the bladder following the resolution of trinitrobenzenesulfonic acid (TNBS) colitis in female rats. Neurourol Urodyn 26:887-893.

Liu F, Takahashi N, Yamaguchi O (2009) Expression of P2X3 purinoceptors in suburothelial myofibroblasts of the normal human urinary bladder. Int J Urol 16:570-575.

Liu HT, Chen CY, Kuo HC (2010) Urinary nerve growth factor levels in overactive bladder syndrome and lower urinary tract disorders. Journal of the Formosan Medical Association = Taiwan yi zhi 109:862-878.

Lu XL, Xu WX, Yan ZY, Qian Z, Xu B, Liu Y, Han LM, Gao RC, Li JN, Yuan M, Zhao CB, Qiao GF, Li BY (2013) Subtype identification in acutely dissociated rat nodose ganglion neurons based on morphologic parameters. International journal of biological sciences 9:716-727.

Lynch AC, Antony A, Dobbs BR, Frizelle FA (2001) Bowel dysfunction following spinal cord injury. Spinal Cord 39:193-203.

Maley B, Elde R (1982) Immunohistochemical localization of putative neurotransmitters within the feline nucleus tractus solitarii. Neuroscience 7:2469-2490.

Maley B, Mullett T, Elde R (1983) The nucleus tractus solitarii of the cat: a comparison of Golgi impregnated neurons with methionine-enkephalin- and substance P-immunoreactive neurons. J Comp Neurol 217:405-417.

Maley BE (1996) Immunohistochemical localization of neuropeptides and neurotransmitters in the nucleus solitarius. Chemical senses 21:367-376.

Maley BE, Newton BW, Howes KA, Herman LM, Oloff CM, Smith KC, Elde RP (1987) Immunohistochemical localization of substance $P$ and enkephalin in the nucleus tractus solitarii of the rhesus monkey, Macaca mulatta. J Comp Neurol 260:483-490.

Malykhina AP (2007) Neural mechanisms of pelvic organ cross-sensitization. Neuroscience 149:660-672.

Malykhina AP, Qin C, Foreman RD, Akbarali HI (2004) Colonic inflammation increases Na+ currents in bladder sensory neurons. Neuroreport 15:2601-2605.

Malykhina AP, Qin C, Greenwood-van Meerveld B, Foreman RD, Lupu F, Akbarali HI (2006) Hyperexcitability of convergent colon and bladder dorsal root ganglion neurons after colonic inflammation: mechanism for pelvic organ cross-talk. Neurogastroenterol Motil 18:936-948.

Malykhina AP, Wyndaele JJ, Andersson KE, De Wachter S, Dmochowski RR (2012) Do the urinary bladder and large bowel interact, in sickness or in health? ICI-RS 2011. Neurourol Urodyn 31:352-358.

Maslany S, Crockett DP, Egger MD (1992) Organization of cutaneous primary afferent fibers projecting to the dorsal horn in the rat: WGA-HRP versus B-HRP. Brain Res 569:123-135.

McAlexander MA, Myers AC, Undem BJ (1999) Adaptation of guinea-pig vagal airway afferent neurones to mechanical stimulation. J Physiol 521 Pt 1:239-247.

Mcllwrath SL, Davis BM, Bielefeldt K (2009) Deletion of P2X3 receptors blunts gastrooesophageal sensation in mice. Neurogastroenterol Motil 21:890-e866. 
McNeal DW, Darling WG, Ge J, Stilwell-Morecraft KS, Solon KM, Hynes SM, Pizzimenti MA, Rotella DL, Vanadurongvan T, Morecraft RJ (2010) Selective long-term reorganization of the corticospinal projection from the supplementary motor cortex following recovery from lateral motor cortex injury. J Comp Neurol 518:586-621.

McNeill DL, Burden HW (1986) Convergence of sensory processes from the heart and left ulnar nerve onto a single afferent perikaryon: a neuroanatomical study in the rat employing fluorescent tracers. Anat Rec 214:441-444, 396-447.

Mendelowitz D, Reynolds PJ, Andresen MC (1995) Heterogeneous functional expression of calcium channels at sensory and synaptic regions in nodose neurons. J Neurophysiol 73:872-875.

Michael GJ, Priestley JV (1999) Differential expression of the mRNA for the vanilloid receptor subtype 1 in cells of the adult rat dorsal root and nodose ganglia and its downregulation by axotomy. J Neurosci 19:1844-1854.

Michl T, Jocic M, Heinemann A, Schuligoi R, Holzer P (2001) Vagal afferent signaling of a gastric mucosal acid insult to medullary, pontine, thalamic, hypothalamic and limbic, but not cortical, nuclei of the rat brain. Pain 92:19-27.

Milby AH, Halpern CH, Baltuch GH (2008) Vagus nerve stimulation for epilepsy and depression. Neurotherapeutics : the journal of the American Society for Experimental NeuroTherapeutics 5:75-85.

Molliver DC, Wright DE, Leitner ML, Parsadanian AS, Doster K, Wen D, Yan Q, Snider WD (1997) IB4-binding DRG neurons switch from NGF to GDNF dependence in early postnatal life. Neuron 19:849-861.

Monnikes H, Ruter J, Konig M, Grote C, Kobelt P, Klapp BF, Arnold R, Wiedenmann B, Tebbe JJ (2003) Differential induction of c-fos expression in brain nuclei by noxious and nonnoxious colonic distension: role of afferent $\mathrm{C}$-fibers and 5-HT3 receptors. Brain Res 966:253-264.

Morrison TC, Dmitrieva N, Winnard KP, Berkley KJ (2006) Opposing viscerovisceral effects of surgically induced endometriosis and a control abdominal surgery on the rat bladder. Fertil Steril 86:1067-1073.

Mullen RJ, Buck CR, Smith AM (1992) NeuN, a neuronal specific nuclear protein in vertebrates. Development 116:201-211.

Murphy PG, Grondin J, Altares M, Richardson PM (1995) Induction of interleukin-6 in axotomized sensory neurons. J Neurosci 15:5130-5138.

Myers AC, Kajekar R, Undem BJ (2002) Allergic inflammation-induced neuropeptide production in rapidly adapting afferent nerves in guinea pig airways. Am J Physiol Lung Cell Mol Physiol 282:L775-781.

Neuhuber WL (1987) Sensory vagal innervation of the rat esophagus and cardia: a light and electron microscopic anterograde tracing study. J Auton Nerv Syst 20:243-255.

Neuhuber WL (1989) Vagal afferent fibers almost exclusively innervate islets in the rat pancreas as demonstrated by anterograde tracing. J Auton Nerv Syst 29:13-18.

Neumann S, Doubell TP, Leslie T, Woolf CJ (1996) Inflammatory pain hypersensitivity mediated by phenotypic switch in myelinated primary sensory neurons. Nature 384:360-364.

Neumann S, Skinner K, Basbaum Al (2005) Sustaining intrinsic growth capacity of adult neurons promotes spinal cord regeneration. Proc Natl Acad Sci U S A 102:16848-16852. 
Nishii H, Nomura M, Fujimoto N, Matsumoto T (2008) Thalamic neural activation in the cyclophosphamide-induced visceral pain model in mice. Neuroscience research 60:219227.

Noronha R, Akbarali H, Malykhina A, Foreman RD, Greenwood-Van Meerveld B (2007) Changes in urinary bladder smooth muscle function in response to colonic inflammation. Am J Physiol Renal Physiol 293:F1461-1467.

North RA (2004) P2X3 receptors and peripheral pain mechanisms. J Physiol 554:301-308.

Novakovic SD, Kassotakis LC, Oglesby IB, Smith JA, Eglen RM, Ford AP, Hunter JC (1999) Immunocytochemical localization of $\mathrm{P} 2 \mathrm{X} 3$ purinoceptors in sensory neurons in naive rats and following neuropathic injury. Pain 80:273-282.

NSCISC (2010) National Spinal Cord Injury Statistical Center. (Chen, Y.-Y., ed) Birmingham.

Ochodnicky P, Cruz CD, Yoshimura N, Michel MC (2011) Nerve growth factor in bladder dysfunction: contributing factor, biomarker, and therapeutic target. Neurourol Urodyn 30:1227-1241.

Oddiah D, Anand P, McMahon SB, Rattray M (1998) Rapid increase of NGF, BDNF and NT-3 mRNAs in inflamed bladder. Neuroreport 9:1455-1458.

Oelrich TM (1983) The striated urogenital sphincter muscle in the female. Anat Rec 205:223-232.

Ohtori S, Takahashi K, Yamagata M, Sameda H, Moriya H, Chiba T, Takahashi Y (2001) Neurones in the dorsal root ganglia of T13, L1 and L2 innervate the dorsal portion of lower lumbar discs in rats. A study using dil, an anterograde neurotracer. J Bone Joint Surg Br 83:11911194.

Okamoto Y, Chaves A, Chen J, Kelley R, Jones K, Weed HG, Gardner KL, Gangi L, Yamaguchi M, Klomkleaw W, Nakayama T, Hamlin RL, Carnes C, Altschuld R, Bauer J, Hai T (2001) Transgenic mice with cardiac-specific expression of activating transcription factor 3 , a stress-inducible gene, have conduction abnormalities and contractile dysfunction. Am J Pathol 159:639-650.

Ortega-Villalobos M, Garcia-Bazan M, Solano-Flores LP, Ninomiya-Alarcon JG, Guevara-Guzman R, Wayner MJ (1990) Vagus nerve afferent and efferent innervation of the rat uterus: an electrophysiological and HRP study. Brain Res Bull 25:365-371.

Ozaki N, Sengupta JN, Gebhart GF (1999) Mechanosensitive properties of gastric vagal afferent fibers in the rat. J Neurophysiol 82:2210-2220.

Page AJ, Martin CM, Blackshaw LA (2002) Vagal mechanoreceptors and chemoreceptors in mouse stomach and esophagus. J Neurophysiol 87:2095-2103.

Pakkenberg B, Gundersen HJ (1988) Total number of neurons and glial cells in human brain nuclei estimated by the disector and the fractionator. Journal of microscopy 150:1-20.

Palea S, Pietra C, Trist DG, Artibani W, Calpista A, Corsi M (1995) Evidence for the presence of both pre- and postjunctional P2-purinoceptor subtypes in human isolated urinary bladder. Br J Pharmacol 114:35-40.

Pan XQ, Gonzalez JA, Chang S, Chacko S, Wein AJ, Malykhina AP (2010) Experimental colitis triggers the release of substance $P$ and calcitonin gene-related peptide in the urinary bladder via TRPV1 signaling pathways. Exp Neurol 225:262-273.

Peng HY, Chang HM, Lee SD, Huang PC, Chen GD, Lai CH, Lai CY, Chiu CH, Tung KC, Lin TB (2008a) TRPV1 mediates the uterine capsaicin-induced NMDA NR2B-dependent cross-organ reflex sensitization in anesthetized rats. Am J Physiol Renal Physiol 295:F1324-1335. 
Peng HY, Chen GD, Tung KC, Lai CY, Hsien MC, Chiu CH, Lu HT, Liao JM, Lee SD, Lin TB (2009) Colon mustard oil instillation induced cross-organ reflex sensitization on the pelvicurethra reflex activity in rats. Pain 142:75-88.

Peng HY, Huang PC, Liao JM, Tung KC, Lee SD, Cheng CL, Shyu JC, Lai CY, Chen GD, Lin TB (2008b) Estrous cycle variation of TRPV1-mediated cross-organ sensitization between uterus and NMDA-dependent pelvic-urethra reflex activity. Am J Physiol Endocrinol Metab 295:E559-568.

Peters JH, Gallaher ZR, Ryu V, Czaja K (2013) Withdrawal and restoration of central vagal afferents within the dorsal vagal complex following subdiaphragmatic vagotomy. J Comp Neurol 521:3584-3599.

Petruska JC, Cooper BY, Gu JG, Rau KK, Johnson RD (2000a) Distribution of P2X1, P2X2, and P2X3 receptor subunits in rat primary afferents: relation to population markers and specific cell types. J Chem Neuroanat 20:141-162.

Petruska JC, Cooper BY, Johnson RD, Gu JG (2000b) Distribution patterns of different P2x receptor phenotypes in acutely dissociated dorsal root ganglion neurons of adult rats. Exp Brain Res 134:126-132.

Petruska JC, Napaporn J, Johnson RD, Cooper BY (2002) Chemical responsiveness and histochemical phenotype of electrophysiologically classified cells of the adult rat dorsal root ganglion. Neuroscience 115:15-30.

Petruska JC, Napaporn J, Johnson RD, Gu JG, Cooper BY (2000c) Subclassified acutely dissociated cells of rat DRG: histochemistry and patterns of capsaicin-, proton-, and ATP-activated currents. J Neurophysiol 84:2365-2379.

Peyronnard JM, Charron L, Messier JP, Lavoie J, Leger C, Faraco-Cantin F (1989) Changes in lectin binding of lumbar dorsal root ganglia neurons and peripheral axons after sciatic and spinal nerve injury in the rat. Cell Tissue Res 257:379-388.

Pezzone MA, Liang R, Fraser MO (2005) A model of neural cross-talk and irritation in the pelvis: implications for the overlap of chronic pelvic pain disorders. Gastroenterology 128:1953-1964.

Phillips C, Monga A (2005) Childbirth and the pelvic floor: "the gynaecological consequences.". Rev Gynaecol Pract 5:15-22.

Phillips RJ, Powley TL (2000) Tension and stretch receptors in gastrointestinal smooth muscle: re-evaluating vagal mechanoreceptor electrophysiology. Brain Res Brain Res Rev 34:126.

Plato M, Kummer W, Haberberger RV (2006) Structural and neurochemical comparison of vagal and spinal afferent neurons projecting to the rat lung. Neurosci Lett 395:215-219.

Popovich PG, Wei P, Stokes BT (1997) Cellular inflammatory response after spinal cord injury in Sprague-Dawley and Lewis rats. J Comp Neurol 377:443-464.

Puizillout JJ, Gambarelli F (1989) Electrophysiological and morphological properties of type C vagal neurons in the nodose ganglion of the cat. J Auton Nerv Syst 29:49-58.

Qiao L, Vizzard MA (2002) Up-regulation of tyrosine kinase (Trka, Trkb) receptor expression and phosphorylation in lumbosacral dorsal root ganglia after chronic spinal cord (T8-T10) injury. J Comp Neurol 449:217-230.

Qiao LY, Grider JR (2007) Up-regulation of calcitonin gene-related peptide and receptor tyrosine kinase TrkB in rat bladder afferent neurons following TNBS colitis. Exp Neurol 204:667679. 
Qiao LY, Vizzard MA (2005) Spinal cord injury-induced expression of TrkA, TrkB, phosphorylated CREB, and c-Jun in rat lumbosacral dorsal root ganglia. J Comp Neurol 482:142-154.

Qin C, Foreman RD, Farber JP (2007) Inhalation of a pulmonary irritant modulates activity of lumbosacral spinal neurons receiving colonic input in rats. Am J Physiol Regul Integr Comp Physiol 293:R2052-2058.

Qin C, Malykhina AP, Akbarali HI, Foreman RD (2005) Cross-organ sensitization of lumbosacral spinal neurons receiving urinary bladder input in rats with inflamed colon. Gastroenterology 129:1967-1978.

Ragnarson B, Bengtsson L, Haegerstrand A (1992) Labeling with fluorescent carbocyanine dyes of cultured endothelial and smooth muscle cells by growth in dye-containing medium. Histochemistry 97:329-333.

Rajendran SK, Reiser JR, Bauman W, Zhang RL, Gordon SK, Korsten MA (1992) Gastrointestinal transit after spinal cord injury: effect of cisapride. Am J Gastroenterol 87:1614-1617.

Randall WC, Milosavljevic M, Wurster RD, Geis GS, Ardell JL (1986) Selective vagal innervation of the heart. Ann Clin Lab Sci 16:198-208.

Randich A, Gebhart GF (1992) Vagal afferent modulation of nociception. Brain Res Brain Res Rev 17:77-99.

Rau KK, Jiang N, Johnson RD, Cooper BY (2007) Heat sensitization in skin and muscle nociceptors expressing distinct combinations of TRPV1 and TRPV2 protein. J Neurophysiol 97:26512662.

Rau KK, Petruska JC, Cooper BY, Johnson RD (2014) Distinct subclassification of DRG neurons innervating the distal colon and glans penis/distal urethra based on the electrophysiological current signature. J Neurophysiol 112:1392-1408.

Rivero-Melian C, Grant G (1991) Choleragenoid horseradish peroxidase used for studying projections of some hindlimb cutaneous nerves and plantar foot afferents to the dorsal horn and Clarke's column in the rat. Exp Brain Res 84:125-132.

Robertson B, Arvidsson J (1985) Transganglionic transport of wheat germ agglutinin-HRP and choleragenoid-HRP in rat trigeminal primary sensory neurons. Brain Res 348:44-51.

Rozman J, Bunc M (2004) Modulation of visceral function by selective stimulation of the left vagus nerve in dogs. Exp Physiol 89:717-725.

Rudick CN, Chen MC, Mongiu AK, Klumpp DJ (2007) Organ cross talk modulates pelvic pain. Am J Physiol Regul Integr Comp Physiol 293:R1191-1198.

Ruggieri SM, Gomez, Amaya S, Braverman A, Lamarre N, Massicotte V, Barbe M (2014) Evidence of vagus nerve sprouting to innervate the urinary bladder and clitoris in a canine model of lower motoneuron lesioned bladder. In: ICS Rio de Janiero.

Sakurai J, Obata K, Ozaki N, Tokunaga A, Kobayashi K, Yamanaka H, Dai Y, Kondo T, Miyoshi K, Sugiura Y, Matsumoto T, Miwa H, Noguchi K (2008) Activation of extracellular signalregulated protein kinase in sensory neurons after noxious gastric distention and its involvement in acute visceral pain in rats. Gastroenterology 134:1094-1103.

Saleem KS, Kondo H, Price JL (2008) Complementary circuits connecting the orbital and medial prefrontal networks with the temporal, insular, and opercular cortex in the macaque monkey. J Comp Neurol 506:659-693.

Samson G, Cardenas DD (2007) Neurogenic bladder in spinal cord injury. Phys Med Rehabil Clin N Am 18:255-274, vi. 
Saper CB, Kibbe MR, Hurley KM, Spencer S, Holmes HR, Leahy KM, Needleman P (1990) Brain natriuretic peptide-like immunoreactive innervation of the cardiovascular and cerebrovascular systems in the rat. Circ Res 67:1345-1354.

Saville LR, Pospisil CH, Mawhinney LA, Bao F, Simedrea FC, Peters AA, O'Connell PJ, Weaver LC, Dekaban GA (2004) A monoclonal antibody to CD11d reduces the inflammatory infiltrate into the injured spinal cord: a potential neuroprotective treatment. Journal of neuroimmunology 156:42-57.

Schachter SC, Saper CB (1998) Vagus nerve stimulation. Epilepsia 39:677-686.

Schild JH, Clark JW, Hay M, Mendelowitz D, Andresen MC, Kunze DL (1994) A- and C-type rat nodose sensory neurons: model interpretations of dynamic discharge characteristics. J Neurophysiol 71:2338-2358.

Schuligoi R, Jocic M, Heinemann A, Schoninkle E, Pabst MA, Holzer P (1998) Gastric acid-evoked $c$-fos messenger RNA expression in rat brainstem is signaled by capsaicin-resistant vagal afferents. Gastroenterology 115:649-660.

Schwartz PJ, De Ferrari GM, Sanzo A, Landolina M, Rordorf R, Raineri C, Campana C, Revera M, Ajmone-Marsan N, Tavazzi L, Odero A (2008) Long term vagal stimulation in patients with advanced heart failure: first experience in man. European journal of heart failure 10:884-891.

Segal JL, Milne N, Brunnemann SR (1995) Gastric emptying is impaired in patients with spinal cord injury. Am J Gastroenterol 90:466-470.

Seki S, Sasaki K, Fraser MO, Igawa Y, Nishizawa O, Chancellor MB, de Groat WC, Yoshimura N (2002) Immunoneutralization of nerve growth factor in lumbosacral spinal cord reduces bladder hyperreflexia in spinal cord injured rats. J Urol 168:2269-2274.

Seki S, Sasaki K, Igawa Y, Nishizawa O, Chancellor MB, De Groat WC, Yoshimura N (2004) Suppression of detrusor-sphincter dyssynergia by immunoneutralization of nerve growth factor in lumbosacral spinal cord in spinal cord injured rats. J Urol 171:478-482.

Sengupta JN, Gebhart GF (1994) Mechanosensitive properties of pelvic nerve afferent fibers innervating the urinary bladder of the rat. J Neurophysiol 72:2420-2430.

Shafik A (1997) Pelvic floor muscles and sphincters during erection and ejaculation. Archives of andrology 39:71-78.

Sharkey KA, Williams RG (1983) Extrinsic innervation of the rat pancreas: demonstration of vagal sensory neurones in the rat by retrograde tracing. Neurosci Lett 42:131-135.

Sherrington SC (1906) The Integrative Action of The Nervous System. New Haven: Yale University Press.

Shoja M. SA, Mirzayan N., Groat C., Watanabe K., Loukas M., Tubbs RS (2013) Neuroanatomy of the female abdominopelvic region: A review with application to pelvic pain syndromes. Clinical Anatomy 26:66-76.

Silva-Ramos M, Silva I, Oliveira O, Ferreira S, Reis MJ, Oliveira JC, Correia-de-Sa P (2013) Urinary ATP may be a dynamic biomarker of detrusor overactivity in women with overactive bladder syndrome. PloS one 8:e64696.

Silverman JD, Kruger L (1990) Selective neuronal glycoconjugate expression in sensory and autonomic ganglia: relation of lectin reactivity to peptide and enzyme markers. J Neurocytol 19:789-801. 
Singh A, Balasubramanian S, Murray M, Lemay M, Houle J (2011) Role of spared pathways in locomotor recovery after body-weight-supported treadmill training in contused rats. J Neurotrauma 28:2405-2416.

Smith SJ, MacDermott AB, Weight FF (1983) Detection of intracellular Ca2+ transients in sympathetic neurones using arsenazo III. Nature 304:350-352.

Springall DR, Cadieux A, Oliveira H, Su H, Royston D, Polak JM (1987) Retrograde tracing shows that CGRP-immunoreactive nerves of rat trachea and lung originate from vagal and dorsal root ganglia. J Auton Nerv Syst 20:155-166.

Spyer KM (1994) Annual review prize lecture. Central nervous mechanisms contributing to cardiovascular control. J Physiol 474:1-19.

Stansfeld CE, Wallis DI (1985) Properties of visceral primary afferent neurons in the nodose ganglion of the rabbit. J Neurophysiol 54:245-260.

Steers WD, Tuttle JB (2006) Mechanisms of Disease: the role of nerve growth factor in the pathophysiology of bladder disorders. Nature clinical practice Urology 3:101-110.

Stinneford JG, Keshavarzian A, Nemchausky BA, Doria MI, Durkin M (1993) Esophagitis and esophageal motor abnormalities in patients with chronic spinal cord injuries. Paraplegia 31:384-392.

Stirling DP, Yong VW (2008) Dynamics of the inflammatory response after murine spinal cord injury revealed by flow cytometry. J Neurosci Res 86:1944-1958.

Stucky CL, Lewin GR (1999) Isolectin B(4)-positive and -negative nociceptors are functionally distinct. J Neurosci 19:6497-6505.

Su X, Wachtel RE, Gebhart GF (1999) Capsaicin sensitivity and voltage-gated sodium currents in colon sensory neurons from rat dorsal root ganglia. Am J Physiol 277:G1180-1188.

Sugiura T, Dang K, Lamb K, Bielefeldt K, Gebhart GF (2005) Acid-sensing properties in rat gastric sensory neurons from normal and ulcerated stomach. J Neurosci 25:2617-2627.

Sui GP, Wu C, Fry CH (2004) Electrical characteristics of suburothelial cells isolated from the human bladder. J Urol 171:938-943.

Sun Y, Keay S, De Deyne PG, Chai TC (2001) Augmented stretch activated adenosine triphosphate release from bladder uroepithelial cells in patients with interstitial cystitis. J Urol 166:1951-1956.

Takahashi R, Yoshizawa T, Yunoki T, Tyagi P, Naito S, de Groat WC, Yoshimura N (2013) Hyperexcitability of bladder afferent neurons associated with reduction of Kv1.4 alphasubunit in rats with spinal cord injury. J Urol 190:2296-2304.

Tanaka M, Uchiyama M, Kitano M (1979) Gastroduodenal disease in chronic spinal cord injuries. An endoscopic study. Archives of surgery (Chicago, III : 1960) 114:185-187.

Tang HB, Li YS, Arihiro K, Nakata Y (2007) Activation of the neurokinin-1 receptor by substance $P$ triggers the release of substance $P$ from cultured adult rat dorsal root ganglion neurons. Mol Pain 3:42.

Theoharides TC, Whitmore K, Stanford E, Moldwin R, O'Leary MP (2008) Interstitial cystitis: bladder pain and beyond. Expert Opin Pharmacother 9:2979-2994.

Titmus MJ, Faber DS (1990) Axotomy-induced alterations in the electrophysiological characteristics of neurons. Prog Neurobiol 35:1-51.

Tong M, Holmes GM (2009) Gastric dysreflexia after acute experimental spinal cord injury in rats. Neurogastroenterol Motil 21:197-206. 
Tong M, Qualls-Creekmore E, Browning KN, Travagli RA, Holmes GM (2011) Experimental spinal cord injury in rats diminishes vagally-mediated gastric responses to cholecystokinin-8s. Neurogastroenterol Motil 23:e69-79.

Torvik A (1956) Afferent connections to the sensory trigeminal nuclei, the nucleus of the solitary tract and adjacent structures; an experimental study in the rat. J Comp Neurol 106:51141.

Tracey KJ (2002) The inflammatory reflex. Nature 420:853-859.

Tracey KJ (2007) Physiology and immunology of the cholinergic antiinflammatory pathway. J Clin Invest 117:289-296.

Traub RJ, Sengupta JN, Gebhart GF (1996) Differential c-fos expression in the nucleus of the solitary tract and spinal cord following noxious gastric distention in the rat. Neuroscience 74:873-884.

Travagli RA, Hermann GE, Browning KN, Rogers RC (2006) Brainstem circuits regulating gastric function. Annu Rev Physiol 68:279-305.

Tsujino H, Kondo E, Fukuoka T, Dai Y, Tokunaga A, Miki K, Yonenobu K, Ochi T, Noguchi K (2000) Activating transcription factor 3 (ATF3) induction by axotomy in sensory and motoneurons: A novel neuronal marker of nerve injury. Mol Cell Neurosci 15:170-182.

Tsuzuki K, Kondo E, Fukuoka T, Yi D, Tsujino H, Sakagami M, Noguchi K (2001) Differential regulation of $\mathrm{P} 2 \mathrm{X}(3)$ mRNA expression by peripheral nerve injury in intact and injured neurons in the rat sensory ganglia. Pain 91:351-360.

Ueno T, Ueno S, Kakazu Y, Akaike N, Nabekura J (2001) Bidirectional modulation of P2X receptor-mediated response by divalent cations in rat dorsal motor nucleus of the vagus neurons. J Neurochem 78:1009-1018.

Undem BJ, Chuaychoo B, Lee MG, Weinreich D, Myers AC, Kollarik M (2004) Subtypes of vagal afferent C-fibres in guinea-pig lungs. J Physiol 556:905-917.

Undem BJ, Weinreich D (1993) Electrophysiological properties and chemosensitivity of guinea pig nodose ganglion neurons in vitro. J Auton Nerv Syst 44:17-33.

Ustinova EE, Fraser MO, Pezzone MA (2006) Colonic irritation in the rat sensitizes urinary bladder afferents to mechanical and chemical stimuli: an afferent origin of pelvic organ cross-sensitization. Am J Physiol Renal Physiol 290:F1478-1487.

Ustinova EE, Fraser MO, Pezzone MA (2010) Cross-talk and sensitization of bladder afferent nerves. Neurourol Urodyn 29:77-81.

Ustinova EE, Gutkin DW, Pezzone MA (2007) Sensitization of pelvic nerve afferents and mast cell infiltration in the urinary bladder following chronic colonic irritation is mediated by neuropeptides. Am J Physiol Renal Physiol 292:F123-130.

van de Merwe JP, Nordling J, Bouchelouche P, Bouchelouche K, Cervigni M, Daha LK, Elneil S, Fall M, Hohlbrugger G, Irwin P, Mortensen S, van Ophoven A, Osborne JL, Peeker R, Richter B, Riedl C, Sairanen J, Tinzl M, Wyndaele JJ (2008) Diagnostic criteria, classification, and nomenclature for painful bladder syndrome/interstitial cystitis: an ESSIC proposal. Eur Urol 53:60-67.

Vanderhorst VG, Mouton L, Blok BF, Holstege G (1996) Distinct cell groups in the lumbosacral cord of the cat project to different areas in the periaqueductal gray. J Comp Neurol 376:361-385.

Verge VM, Merlio JP, Grondin J, Ernfors P, Persson H, Riopelle RJ, Hokfelt T, Richardson PM (1992) Colocalization of NGF binding sites, trk mRNA, and low-affinity NGF receptor 
mRNA in primary sensory neurons: responses to injury and infusion of NGF. J Neurosci 12:4011-4022.

Versi E, Cardozo LD, Studd JW, Brincat M, O'Dowd TM, Cooper DJ (1986) Internal urinary sphincter in maintenance of female continence. Br Med J (Clin Res Ed) 292:166-167.

Vilensky JA, Bell DR, Gilman S (2004) "On the physiology of micturition" by Denny-Brown and Robertson: a classic paper revisited. Urology 64:182-186.

Vizzard MA (1997) Increased expression of neuronal nitric oxide synthase in bladder afferent and spinal neurons following spinal cord injury. Developmental neuroscience 19:232246.

Vizzard MA (1999) Alterations in growth-associated protein (GAP-43) expression in lower urinary tract pathways following chronic spinal cord injury. Somatosens Mot Res 16:369-381.

Vizzard MA (2000a) Changes in urinary bladder neurotrophic factor mRNA and NGF protein following urinary bladder dysfunction. Exp Neurol 161:273-284.

Vizzard MA (2000b) Increased expression of spinal cord Fos protein induced by bladder stimulation after spinal cord injury. Am J Physiol Regul Integr Comp Physiol 279:R295305.

Vizzard MA (2001) Alterations in neuropeptide expression in lumbosacral bladder pathways following chronic cystitis. J Chem Neuroanat 21:125-138.

Vizzard MA (2006) Neurochemical plasticity and the role of neurotrophic factors in bladder reflex pathways after spinal cord injury. Prog Brain Res 152:97-115.

Vizzard MA, Brisson M, de Groat WC (2000) Transneuronal labeling of neurons in the adult rat central nervous system following inoculation of pseudorabies virus into the colon. Cell Tissue Res 299:9-26.

Vlaskovska M, Kasakov L, Rong W, Bodin P, Bardini M, Cockayne DA, Ford AP, Burnstock G (2001) P2X3 knock-out mice reveal a major sensory role for urothelially released ATP. J Neurosci 21:5670-5677.

Vulchanova L, Arvidsson U, Riedl M, Wang J, Buell G, Surprenant A, North RA, Elde R (1996) Differential distribution of two ATP-gated channels ( $P 2 X$ receptors) determined by immunocytochemistry. Proc Natl Acad Sci U S A 93:8063-8067.

Vulchanova L, Olson TH, Stone LS, Riedl MS, Elde R, Honda CN (2001) Cytotoxic targeting of isolectin IB4-binding sensory neurons. Neuroscience 108:143-155.

Vulchanova L, Riedl MS, Shuster SJ, Buell G, Surprenant A, North RA, Elde R (1997) Immunohistochemical study of the P2X2 and P2X3 receptor subunits in rat and monkey sensory neurons and their central terminals. Neuropharmacology 36:1229-1242.

Vulchanova L, Riedl MS, Shuster SJ, Stone LS, Hargreaves KM, Buell G, Surprenant A, North RA, Elde R (1998) P2X3 is expressed by DRG neurons that terminate in inner lamina II. Eur J Neurosci 10:3470-3478.

Wakabayashi Y, Tomoyoshi T, Fujimiya M, Arai R, Maeda T (1993) Substance P-containing axon terminals in the mucosa of the human urinary bladder: pre-embedding immunohistochemistry using cryostat sections for electron microscopy. Histochemistry 100:401-407.

Wang G, Scott SA (1999) Independent development of sensory and motor innervation patterns in embryonic chick hindlimbs. Dev Biol 208:324-336. 
Wang HZ, Li SR, Wen C, Xiao CG, Su BY (2007) Morphological changes of cholinergic nerve fibers in the urinary bladder after establishment of artificial somatic-autonomic reflex arc in rats. Neurosci Bull 23:277-281.

Wang L, Feng D, Yan H, Wang Z, Pei L (2014) Comparative analysis of P2X1, P2X2, P2X3, and $\mathrm{P} 2 \mathrm{X} 4$ receptor subunits in rat nodose ganglion neurons. PloS one 9:e96699.

Wang T, Molliver DC, Jing X, Schwartz ES, Yang FC, Samad OA, Ma Q, Davis BM (2011) Phenotypic switching of nonpeptidergic cutaneous sensory neurons following peripheral nerve injury. PloS one 6:e28908.

Wang YH, Tache Y, Sheibel AB, Go VL, Wei JY (1997) Two types of leptin-responsive gastric vagal afferent terminals: an in vitro single-unit study in rats. Am J Physiol 273:R833-837.

Wank M, Neuhuber WL (2001) Local differences in vagal afferent innervation of the rat esophagus are reflected by neurochemical differences at the level of the sensory ganglia and by different brainstem projections. J Comp Neurol 435:41-59.

Ward PJ, Herrity AN, Smith RR, Willhite A, Harrison BJ, Petruska JC, Harkema SJ, Hubscher CH (2014) Novel multi-system functional gains via task specific training in spinal cord injured male rats. J Neurotrauma 31:819-833.

Whorwell PJ, Lupton EW, Erduran D, Wilson K (1986a) Bladder smooth muscle dysfunction in patients with irritable bowel syndrome. Gut 27:1014-1017.

Whorwell PJ, McCallum M, Creed FH, Roberts CT (1986b) Non-colonic features of irritable bowel syndrome. Gut 27:37-40.

Widdicombe JG (1954) The site of pulmonary stretch receptors in the cat. J Physiol 125:336-351.

Williams RE, 3rd, Bauman WA, Spungen AM, Vinnakota RR, Farid RZ, Galea M, Korsten MA (2012) SmartPill technology provides safe and effective assessment of gastrointestinal function in persons with spinal cord injury. Spinal Cord 50:81-84.

Winnard KP, Dmitrieva N, Berkley KJ (2006) Cross-organ interactions between reproductive, gastrointestinal, and urinary tracts: modulation by estrous stage and involvement of the hypogastric nerve. Am J Physiol Regul Integr Comp Physiol 291:R1592-1601.

Wiseman OJ, Fowler CJ, Landon DN (2003) The role of the human bladder lamina propria myofibroblast. BJU Int 91:89-93.

Wolf C, Meiners TH (2003) Dysphagia in patients with acute cervical spinal cord injury. Spinal Cord 41:347-353.

Wu XY, Zhu JX, Gao J, Owyang C, Li Y (2005) Neurochemical phenotype of vagal afferent neurons activated to express C-FOS in response to luminal stimulation in the rat. Neuroscience 130:757-767.

Wyndaele JJ (1991) Investigation of the afferent nerves of the lower urinary tract in patients with 'complete' and 'incomplete' spinal cord injury. Paraplegia 29:490-494.

Wyndaele JJ (1997) Correlation between clinical neurological data and urodynamic function in spinal cord injured patients. Spinal Cord 35:213-216.

Xiang Z, Bo X, Oglesby I, Ford A, Burnstock G (1998) Localization of ATP-gated P2X2 receptor immunoreactivity in the rat hypothalamus. Brain Res 813:390-397.

Yiangou Y, Facer P, Ford A, Brady C, Wiseman O, Fowler CJ, Anand P (2001) Capsaicin receptor VR1 and ATP-gated ion channel P2X3 in human urinary bladder. BJU Int 87:774-779.

Yokokawa K, Tohyama M, Shiosaka S, Shiotani Y, Sonoda T, Emson PC, Hillyard CV, Girgis S, Maclntyre I (1986) Distribution of calcitonin gene-related peptide-containing fibers in the urinary bladder of the rat and their origin. Cell Tissue Res 244:271-278. 
Yoshimura N (1999) Bladder afferent pathway and spinal cord injury: possible mechanisms inducing hyperreflexia of the urinary bladder. Prog Neurobiol 57:583-606.

Yoshimura N, de Groat WC (1997) Plasticity of $\mathrm{Na}+$ channels in afferent neurones innervating rat urinary bladder following spinal cord injury. J Physiol 503 ( Pt 2):269-276.

Yoshimura N, Erdman SL, Snider MW, de Groat WC (1998) Effects of spinal cord injury on neurofilament immunoreactivity and capsaicin sensitivity in rat dorsal root ganglion neurons innervating the urinary bladder. Neuroscience 83:633-643.

Yoshimura N, Seki S, Erickson KA, Erickson VL, Hancellor MB, de Groat WC (2003) Histological and electrical properties of rat dorsal root ganglion neurons innervating the lower urinary tract. J Neurosci 23:4355-4361.

Yoshimura N, White G, Weight FF, de Groat WC (1994) Patch-clamp recordings from subpopulations of autonomic and afferent neurons identified by axonal tracing techniques. J Auton Nerv Syst 49:85-92.

Yoshimura N, White G, Weight FF, de Groat WC (1996) Different types of Na+ and A-type K+ currents in dorsal root ganglion neurones innervating the rat urinary bladder. J Physiol 494 ( Pt 1):1-16.

Young RL, Cooper NJ, Blackshaw LA (2008) Chemical coding and central projections of gastric vagal afferent neurons. Neurogastroenterol Motil 20:708-718.

Zhang X, Ji RR, Arvidsson J, Lundberg JM, Bartfai T, Bedecs K, Hokfelt T (1996) Expression of peptides, nitric oxide synthase and NPY receptor in trigeminal and nodose ganglia after nerve lesions. Exp Brain Res 111:393-404.

Zhong F, Christianson JA, Davis BM, Bielefeldt K (2008) Dichotomizing axons in spinal and vagal afferents of the mouse stomach. Dig Dis Sci 53:194-203.

Zhou XF, Chie ET, Deng YS, Zhong JH, Xue Q, Rush RA, Xian CJ (1999) Injured primary sensory neurons switch phenotype for brain-derived neurotrophic factor in the rat. Neuroscience 92:841-853.

Zhuo H, Helke CJ (1996) Presence and localization of neurotrophin receptor tyrosine kinase (TrkA, TrkB, TrkC) mRNAs in visceral afferent neurons of the nodose and petrosal ganglia. Brain research Molecular brain research 38:63-70.

Zhuo H, Ichikawa H, Helke CJ (1997) Neurochemistry of the nodose ganglion. Prog Neurobiol 52:79-107.

Zinck ND, Downie JW (2008) IB4 afferent sprouting contributes to bladder dysfunction in spinal rats. Exp Neurol 213:293-302.

Zinck ND, Rafuse VF, Downie JW (2007) Sprouting of CGRP primary afferents in lumbosacral spinal cord precedes emergence of bladder activity after spinal injury. Exp Neurol 204:777-790.

Zvara P, Braas KM, May V, Vizzard MA (2006) A role for pituitary adenylate cyclase activating polypeptide (PACAP) in detrusor hyperreflexia after spinal cord injury (SCI). Ann N Y Acad Sci 1070:622-628.

Zvarova K, Dunleavy JD, Vizzard MA (2005) Changes in pituitary adenylate cyclase activating polypeptide expression in urinary bladder pathways after spinal cord injury. Exp Neurol 192:46-59.

Zvarova K, Murray E, Vizzard MA (2004) Changes in galanin immunoreactivity in rat lumbosacral spinal cord and dorsal root ganglia after spinal cord injury. J Comp Neurol 475:590-603. 
CURRICULUM VITAE

APRIL N. HERRITY, MS, DC University of Louisville 511 South Floyd MDR Building, Room 531

Louisville, KY 40202

Office: 502-852-7201 Cell: 502-424-6338

anherr02@louisville.edu

\section{PRESENT POSITION}

Graduate PhD candidate

Anatomical Sciences and Neurobiology

University of Louisville, $\mathrm{KY}$

Expected Graduation: December 2014

\section{EDUCATION}

2011 MS Anatomical Sciences and Neurobiology, University of Louisville, KY Mentor: Charles Hubscher, PhD

2003 DC Cleveland Chiropractic College, Los Angeles, CA

1998 BS Bilingual Education, University of New Mexico, Albuquerque, NM

\section{PROFESSIONAL EXPERIENCE}

2014 - Present Graduate Student Committee Chair for selecting the keynote speaker for the 2015 Kentucky Spinal Cord and Head Injury Research Trust Symposium

2013 - $2014 \quad$ Mentor, Manual High School student science fair project

2011 - $2014 \quad$ School of Medicine Graduate Council Student

Representative

2010 - $2013 \quad$ Teaching Assistant, Neuroanatomy, University of Louisville

2009 - Present Graduate Fellow, University of Louisville 
2003 - Present

$2000-2002$

2012-2015
Chiropractor

Assistant Instructor, Kinetic Palpation and Adjusting Lab, Cleveland Chiropractic College, Los Angeles, CA

\section{FUNDING}

F31 Ruth L. Kirschstein National Research Service Award (NRSA), "Effects of spinal cord injury on vagal afferents from the rat bladder and colon."

\section{PUBLICATIONS}

Herrity AN, Petruska JC, Rau KK, Stirling DP, Hubscher CH (2014). The effect of spinal cord injury on the neurochemical properties of vagal neurons. (Submitted, American Journal of Physiology)

Herrity AN, Rau KK, Petruska JC, Stirling DP, Hubscher CH (2014). Identification of bladder and colon afferents in the nodose ganglia of male rats. J Comp Neurol. Nov 1;522(16):3667-82.

Ward PJ, Herrity AN, Smith RR, Willhite A, Harrison BJ, Petruska JC, Harkema $\mathrm{SJ}$, and Hubscher $\mathrm{CH}$ (2013). Novel multi-system functional gains via task specific training in spinal cord injured male rats. J Neurotrauma. May 1;31(9):819-33.

\section{ABSTRACTS}

Herrity AN, Petruska JC, Rau KK, Stirling DP, Hubscher CH (2014). The effect of spinal cord injury on bladder-specific nodose ganglion neurons. 422.11/Y6 SFN, Washington D.C.

Herrity AN, Petruska JC, Rau KK, Stirling DP, Hubscher CH (2014). The effect of spinal cord injury on the neurochemical properties of vagal neurons. Research Louisville.

Herrity AN, Rau KK, Petruska JC, Stirling DP, Hubscher CH (2013). Is the vagus an alternate pathway from the pelvic viscera to the brainstem? 831.26/LL5 SFN San Diego.

Hubscher CH, Herrity AN, Rau KK, Petruska JC (2013). Impact of spinal cord injury on vagal afferents. 831.29/LL8 SFN San Diego. 
Herrity AN, Rau KK, Petruska JC, Stirling DP, Hubscher CH (2013). Spinal and vagal afferent innervation of the male rat urinary bladder and distal colon. Research Louisville.

Herrity AN, Rau KK, Petruska JC, Hubscher CH (2013). The identification and neurochemical profile of bladder and colon vagal afferents in the male rat. Kentucky Spinal Cord Head Injury Research Trust Symposium, Louisville, KY

Herrity AN, Rau KK, Petruska JC, Hubscher CH (2013). The identification and neurochemical profile of bladder and colon vagal afferents in the male rat. Neuroscience Day, University of Louisville

Herrity AN, Ward PJ, Harkema SJ, Hubscher CH (2012). Locomotor training time affects at-level allodynia in a rodent model of spinal cord injury. 85.18/NN8, SFN New Orleans, LA.

Herrity AN, Ward PJ, Harkema SJ, Hubscher CH (2012). Locomotor training duration impacts at-level allodynia in a rodent model of spinal cord injury. Research Louisville.

Herrity AN, Ward PJ, Harkema SJ, Hubscher CH (2012). Locomotor training ameliorates spinal cord injury induced at-level hypersensitivity. Neuroscience Day, University of Louisville.

Ward PJ, Shah CN, Herrity AN, Atkinson DA, Stewart BR, Harkema SJ, Hubscher $\mathrm{CH}$ (2012). Step training improves overground locomotion following moderate contusion in rats: open field scoring, kinematics, and gait analysis. 85.17/NN7, SFN New Orleans, LA.

Ward PJ, Herrity AN, Hubscher CH (2011) Polyuria in a rat model of contusive spinal cord injury.160.06/CC5, SFN Washington, D.C.

Herrity AN, Ward PJ, Harkema SJ, Hubscher CH (2010) Body Weight Supported Treadmill Training Decreases At-Level Allodynia Following Spinal Cord Injury in Male Rats. 681.17/TT6, SFN San Diego, CA.

\section{PRESENTATIONS}

2014 Neuroscience Day, UofL, invited speaker, "The impact of spinal cord injury on vagal afferents."

$2013 \quad$ Neuroscience Day "Data Blitz" invited presenter. 
2013

2010-Present

2006

2006

2005

2010-2011

2010-Present

2010-Present

2013

2012

2012

2012

2009-2011

2003

2003

2003

2003

2002

1999-2003

1998

1994-1998
Kentucky Spinal Cord Head Injury Research Trust

Symposium, poster presentation

Research Louisville and Neuroscience Day, poster presentations

Louisville Ballet School invited Speaker, Topic: Performance and Nutrition, March and September.

Sisters of Charity of Nazareth, Bardstown KY, invited speaker, Topic: Complementary and Alternative Medicine. Curves Gym for Women, invited Speaker, Topic: Instructor Training: Low back cervical spine conditions affecting circuit training.

\section{SEMINARS/CONFERENCES}

Graduate Teaching Academy Training, University of Louisville

Kentucky Spinal Cord and Head Injury Research Trust Symposium

Society for Neuroscience

\section{SCHOLARSHIPSIAWARDS}

Third Place, Poster presentation at Research Louisville First Place, Poster presentation at Neuroscience Day, UofL Fall Student Spotlight Recipient, Kentucky Spinal Cord and Injury Research Center, University of Louisville Student of the Month, School of Interdisciplinary Graduate Studies, University of Louisville

Second Place, Poster presentation at Neuroscience Day, University of Louisville Graduate Fellowship Awarded, University of Louisville Valedictorian, Cleveland Chiropractic College, Los Angeles Clinic Excellence Award, Cleveland Chiropractic College Summa Cum Laude, Cleveland Chiropractic College Dr. Carl Cleveland III, Tuition Grant, Cervical Spine Syndromes

Department of Nutrition, Tuition Grant, Benefits of Flaxseed Dean's List, Cleveland Chiropractic College Summa Cum Laude graduate, University of New Mexico Full Academic-Soccer Scholarship, University of New Mexico 


\section{AFFLIATIONS}

2009-Present

2009-Present

2005-2009

2013

2012

2010

$2005-2009$
Society for Neuroscience, Member

Local Chapter Society for Neuroscience, Member, Louisville Official Chiropractor for The Louisville Ballet

\section{COMMUNITY SERVICE}

Frazier Rehab Institute/Kentucky One Health "Casino Night", fundraiser to benefit Spinal Cord Medicine Program, Nov 2. Frazier Rehab Institute/Kentucky One Health "Casino Night", Sept 14.

Susan G Komen, "Pink Tie Lounge" Fundraiser, Committee Member, Louisville, KY, September.

Louisville Ballet, Chiropractic and Rehabilitation services provided. 